University of Tennessee Health Science Center

UTHSC Digital Commons

\title{
Assessing Neuronal Synchrony and Brain Function Through Local Field Potential and Spike Analysis
}

\author{
Samuel Stuart McAfee \\ University of Tennessee Health Science Center
}

Follow this and additional works at: https://dc.uthsc.edu/dissertations

Part of the Medical Neurobiology Commons

\section{Recommended Citation}

McAfee, Samuel Stuart (http://orcid.org/0000-0003-4121-0592), "Assessing Neuronal Synchrony and Brain Function Through Local Field Potential and Spike Analysis" (2017). Theses and Dissertations (ETD). Paper 448. http://dx.doi.org/10.21007/etd.cghs.2017.0445. 


\title{
Assessing Neuronal Synchrony and Brain Function Through Local Field Potential and Spike Analysis
}

\author{
Abstract \\ Studies of neuronal network oscillations and rhythmic neuronal synchronization have led to a number of \\ important insights in recent years, giving us a better understanding of the temporal organization of \\ neuronal activity related to essential brain functions like sensory processing and cognition. Important \\ principles and theories have emerged from these findings, including the communication through \\ coherence hypothesis, which proposes that synchronous oscillations render neuronal communication \\ effective, selective, and precise. The implications of such a theory may be universal for brain function, as \\ the determinants of neuronal communication inextricably shape the neuronal representation of \\ information in the brain. However, the study of communication through coherence is still relatively young. \\ Since its articulation in 2005 , the theory has predominantly been applied to assess cortical function and \\ its communication with downstream targets in different sensory and behavioral conditions. The results \\ herein are intended to bolster this hypothesis and explore new ways in which oscillations coordinate \\ neuronal communication in distributed regions. This includes the development of new analytic tools for \\ interpreting electrophysiological patterns, inspired by phase synchronization and spike train analysis. \\ These tools aim to offer fast results with clear statistical and physiological interpretation.

\section{Document Type} \\ Dissertation \\ Degree Name \\ Doctor of Philosophy (PhD)

\section{Program} \\ Biomedical Sciences \\ Research Advisor \\ Detlef H. Heck, Ph.D. \\ Keywords \\ Coherence, Oscillations, Phase Synchronization, Respiration Oscillations, Thalamocortical Transmission \\ Subject Categories \\ Medical Neurobiology | Medical Sciences | Medicine and Health Sciences
}




\title{
Assessing Neuronal Synchrony and Brain Function Through Local Field Potential and Spike Analysis
}

\author{
A Dissertation \\ Presented for \\ The Graduate Studies Council \\ The University of Tennessee \\ Health Science Center
}

In Partial Fulfillment

Of the Requirements for the Degree

Doctor of Philosophy

From The University of Tennessee

By

Samuel Stuart McAfee

December 2017 
Portions of Chapter 5 (C) 2016 by Elsevier BV. Portions of Chapter 5 (C) 2017 by Nature Publishing Group. Copyright (C) 2017 by Samuel Stuart McAfee.

All rights reserved. 


\section{DEDICATION}

To my family and friends, who are entirely excellent.

To my beautiful wife, Gal, for her endless love and support.

And to my dog, Bucky, for being a good boy. 


\section{ACKNOWLEDGEMENTS}

First and foremost, I would like to thank my advisor, Dr. Detlef Heck, and all of our lab members for their invaluable help and guidance throughout the course of this project. In particular, I would like to thank Dr. Heck for offering me endless encouragement to discover which questions I was most interested in, and the freedom to find the answers in my own way. Thank you Dr. Yu Liu, for your patience and diligence every day, and for teaching me how to perform experiments properly. And lastly, thank you Shuhua Qi for being so helpful and making sure we always had the right tools at hand.

I would also like to thank the members of my graduate committee for their help in focusing my project: Dr. Robert Foehring, Dr. Robert Ogg, Dr. Shalini Narayana, and Dr. Max Fletcher. Additionally I would like to thank the Neuroscience Institute for its generous support with my graduate fellowship.

Lastly, I would like to thank the students within the graduate program for being a constant source of support, curiosity, and constructive criticism. I would like to offer a special thanks in that regard to Kevin Hope, Matthew Kirchner, Zachary Goldsmith, and Cameron Ogg. 


\begin{abstract}
Studies of neuronal network oscillations and rhythmic neuronal synchronization have led to a number of important insights in recent years, giving us a better understanding of the temporal organization of neuronal activity related to essential brain functions like sensory processing and cognition. Important principles and theories have emerged from these findings, including the communication through coherence hypothesis, which proposes that synchronous oscillations render neuronal communication effective, selective, and precise. The implications of such a theory may be universal for brain function, as the determinants of neuronal communication inextricably shape the neuronal representation of information in the brain. However, the study of communication through coherence is still relatively young. Since its articulation in 2005, the theory has predominantly been applied to assess cortical function and its communication with downstream targets in different sensory and behavioral conditions. The results herein are intended to bolster this hypothesis and explore new ways in which oscillations coordinate neuronal communication in distributed regions. This includes the development of new analytic tools for interpreting electrophysiological patterns, inspired by phase synchronization and spike train analysis. These tools aim to offer fast results with clear statistical and physiological interpretation.
\end{abstract}




\section{TABLE OF CONTENTS}

\section{CHAPTER 1. INTERPRETATION AND SIGNIFICANCE OF ELECTROPHYSIOLOGICAL RECORDINGS........................................................1}

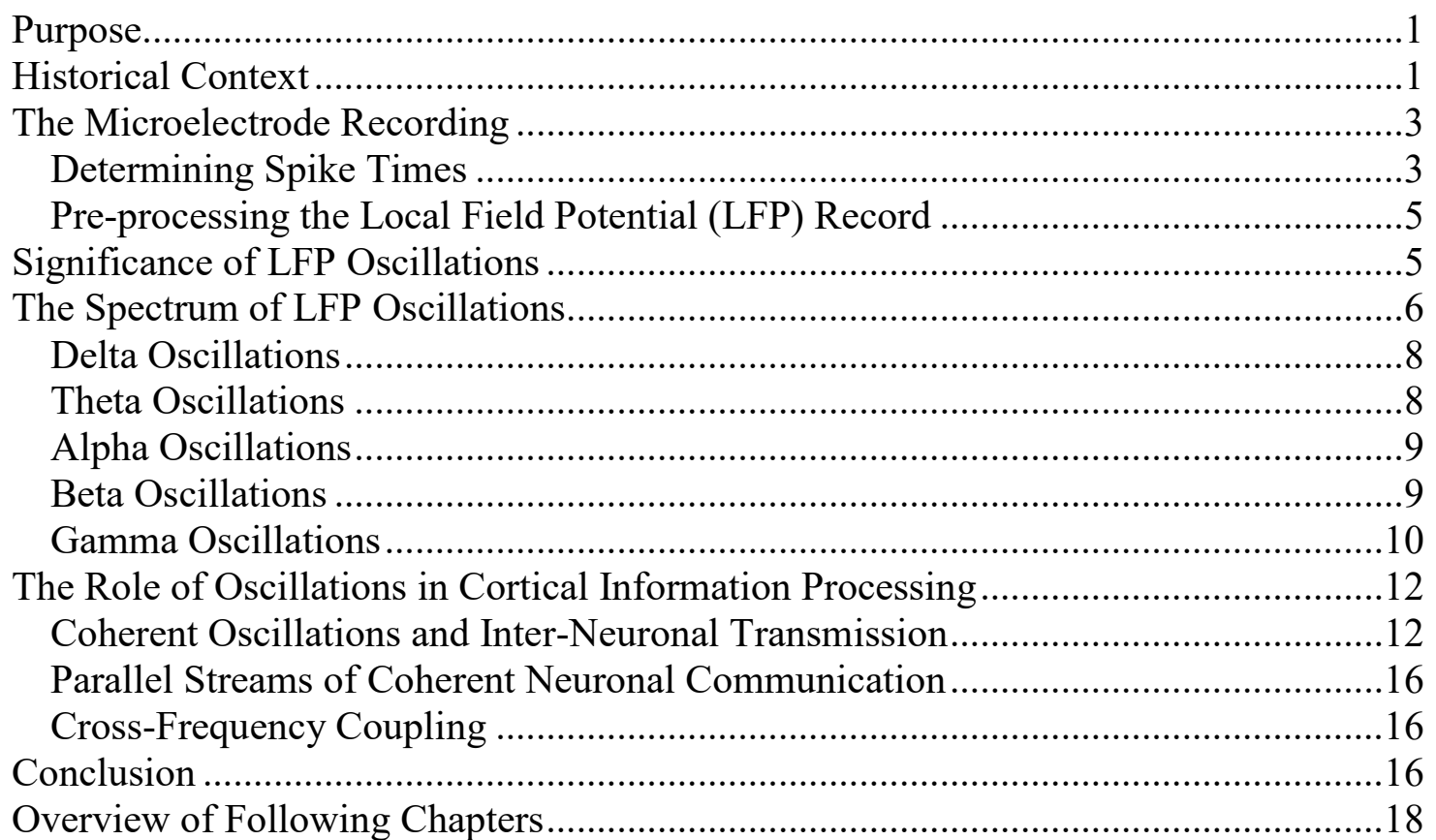

\section{CHAPTER 2. DEVELOPMENT OF NOVEL METHODS FOR ASSESSING NEURONAL SYNCHRONY AND BRAIN FUNCTION THROUGH LFP AND SPIKE ANALYSIS}

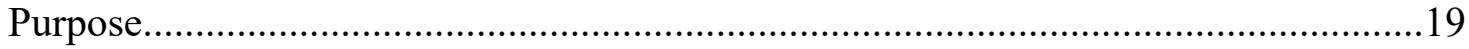

LFP Frequency, Phase and Amplitude Estimation ………….....................................19

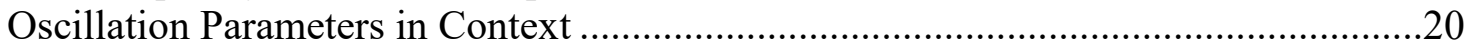

The Synchronization Metric $r$ and Its Statistical Evaluation......................................22

Parametric Statistical Tests Using the $r$ Value …………......................................23

Evaluation of Binned Test Sensitivity and Accuracy .............................................28

Application to LFP Phase Synchronization Analysis ………………………..........28

Oscillatory Changes in Spike Synchrony ……………...................................................31

Creating the Spike Synchrony Function ......................................................................

Statistical Evaluation of Rhythmic Spike Synchrony and CTC .................................34

Phase-Relationship Tuning of Spike Activity ……….................................................37

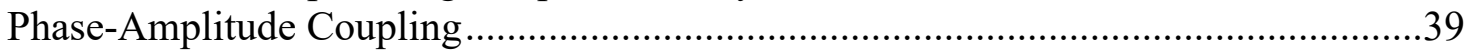

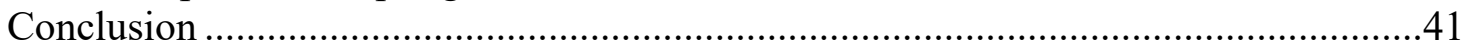

\section{CHAPTER 3. SYNCHRONOUS OSCILLATIONS AND}

THALAMOCORTICAL TRANSMISSION IN THE MOUSE VISUAL

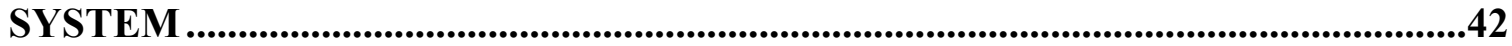

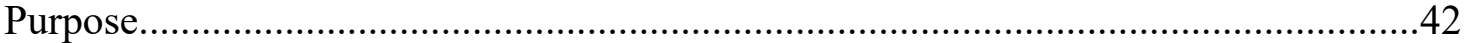

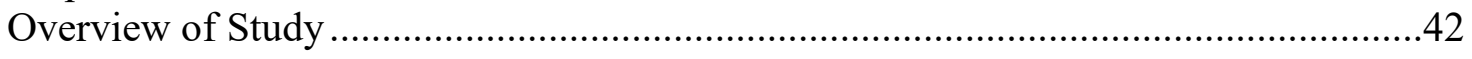




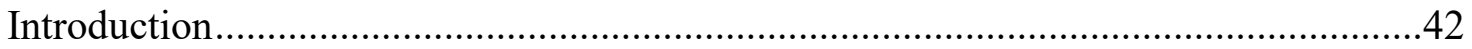

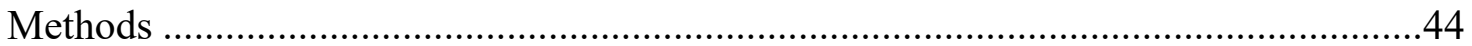

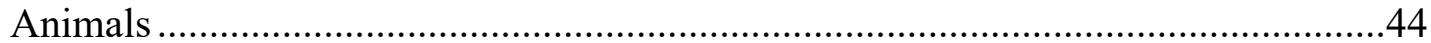

Preparation for Awake, Multisite Recordings ......................................................44

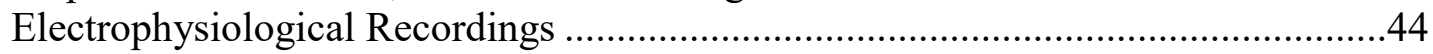

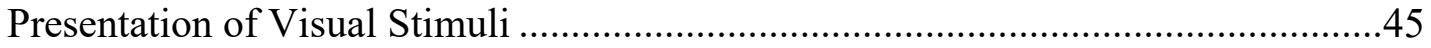

Analysis of Local Field Potential Amplitude and Phase ……………………...........46

Time Resolved LGN-V1 Spike Synchrony .............................................................46

Correlation of Spike Synchrony and Gamma Activity .............................................4

Experimental Design and Statistical Analysis ......................................................4

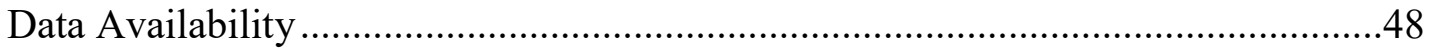

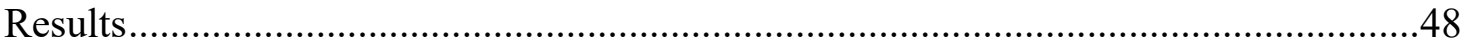

Visual Stimulus-Driven Gamma Oscillations in dLGN and Layer 4 of V1 ..............48

Phase Synchronization and Amplitude of LFP Oscillations ........................................48

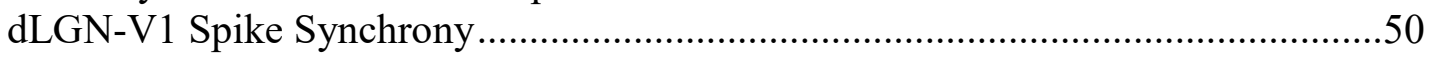

dLGN-V1 Spike Synchrony and V1 Oscillations .................................................50

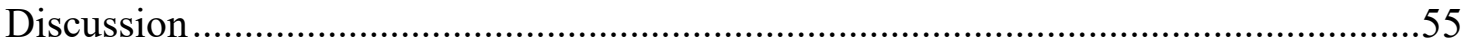

Comparison to Awake Primates................................................................................55

Broad Synchrony between Thalamic and Visual Cortical Units ................................56

Communication through Coherence (CTC) in Thalamocortical Transmission..........57

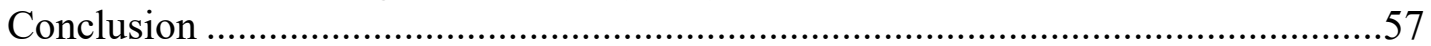

\section{CHAPTER 4. CEREBELLAR PURKINJE CELL TUNING TO PHASE DIFFERENCES BETWEEN THE MEDIAL PREFRONTAL CORTEX AND HIPPOCAMPUS IN MICE ...............................................................................59}

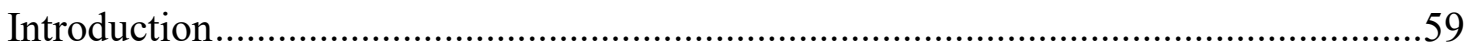

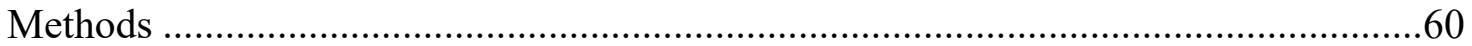

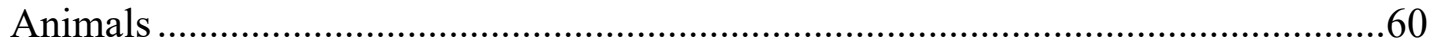

Preparation for Head-Fixed Recording .............................................................60

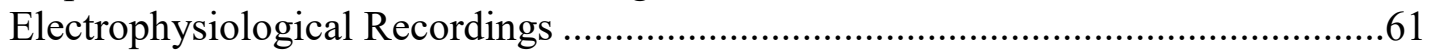

Analysis of PC Single-Unit Spike Data ..............................................................63

Pre-Preprocessing of LFPs..................................................................................63

Estimation of LFP Phase Relationships and Corresponding Spikes............................64

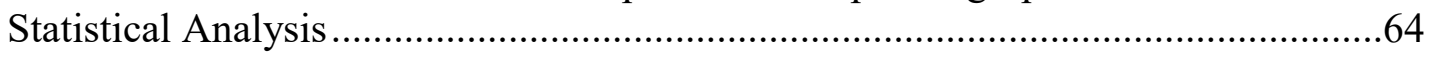

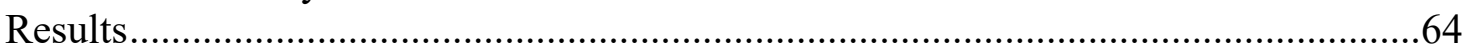

Link between PFC-CA1 Phase Differences and Spike Rate in Individual PCs ........64 PC Activity Modulation Occurs across the Frequency Spectrum of PFC/CA1

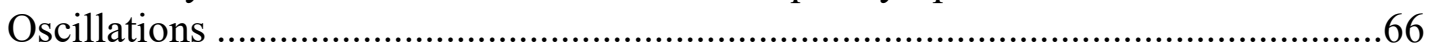

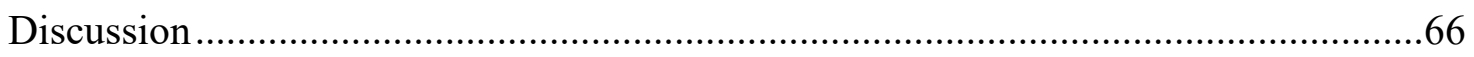

\section{CHAPTER 5. RESPIRATION AS A FUNDAMENTAL RHYTHM OF BRAIN} FUNCTION

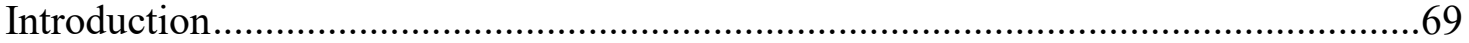

Development of an Effective Method for Measurement of Respiration in Mice ...........72

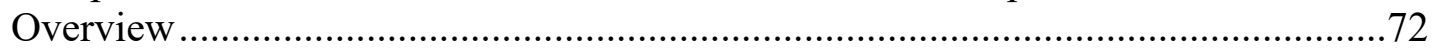

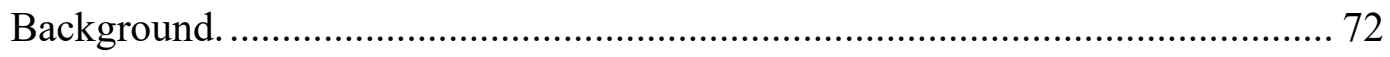




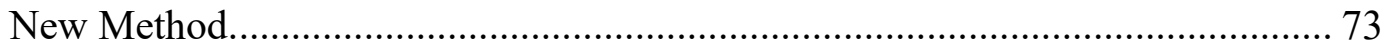

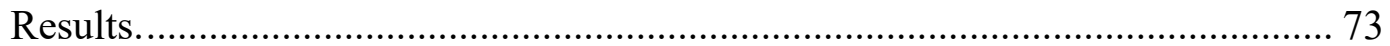

Comparison with Existing Methods............................................................... 73

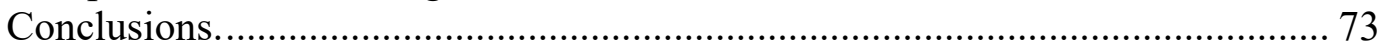

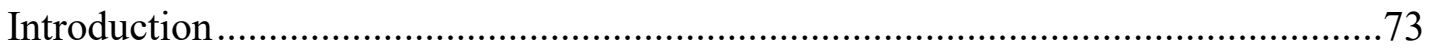

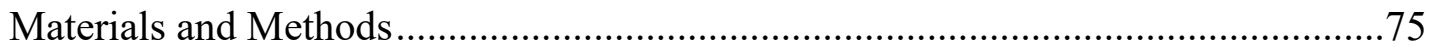

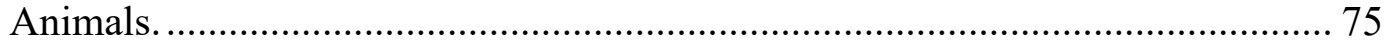

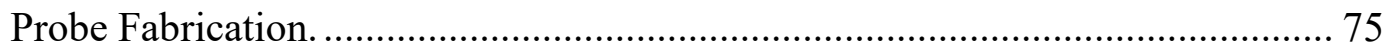

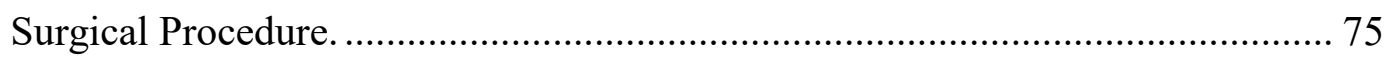

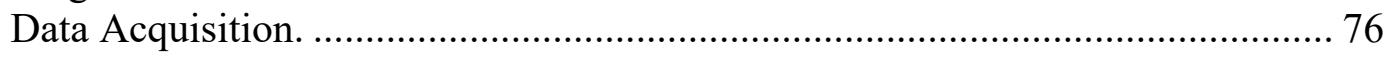

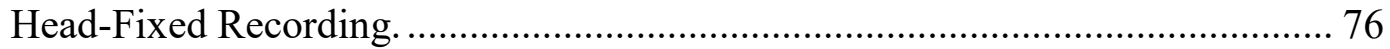

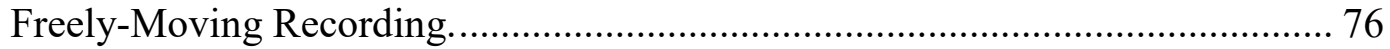

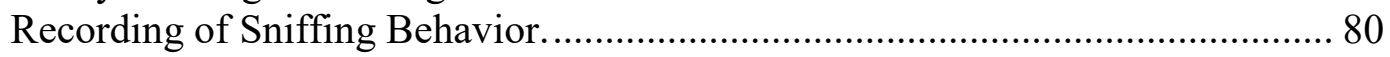

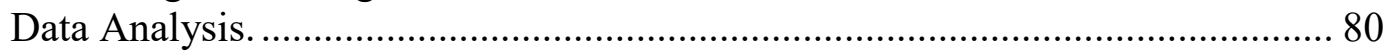

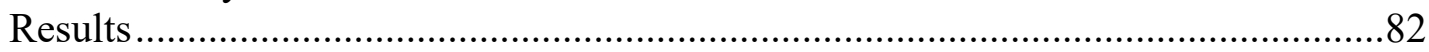

Comparison of Head-Fixed Recording Methods.............................................. 82

Comparison of Freely-Moving Recording Methods............................................. 82

Assessment of Sniffing Behavior....................................................................... 82

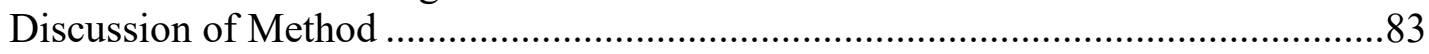

Respiratory Modulation of LFPs and Spikes in the Mouse Visual System and

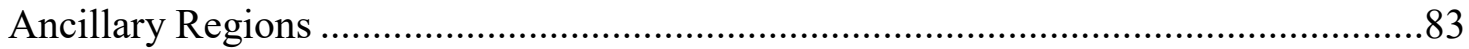

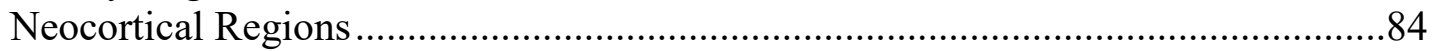

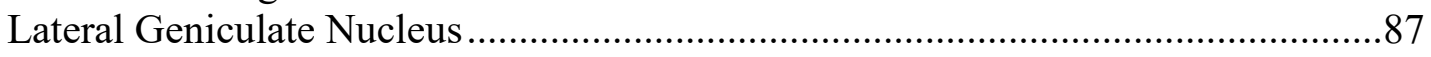

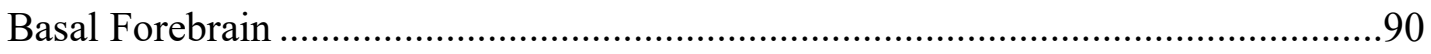

Disruption of Thalamocortical Phase Synchronization and Visual Acuity by OB

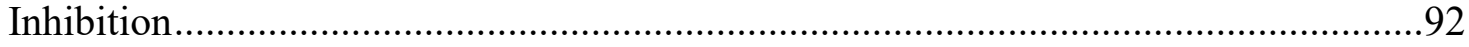

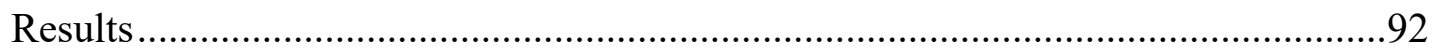

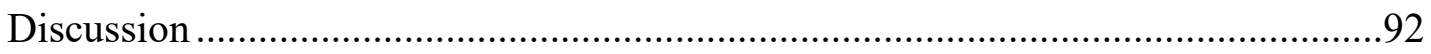

Respiratory Entrainment of Hippocampal Sharp-Wave Ripple Activity in CA1 ..........95

Results....................................................................................................96

Effect of Inhibiting Respiration-Locked OB Activity on Respiration-Locked

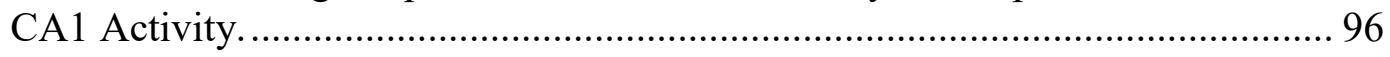

Effect of Respiration-Locked OB Activity SWR Event Timing. ........................ 100

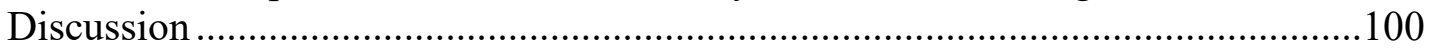

Respiratory Modulation of Electrocorticography in Humans.....................................102

Respiration-Locked Slow Oscillations ................................................................103

Amplitude Coupling of Fast ECoG Oscillations to the Respiratory Phase .............103

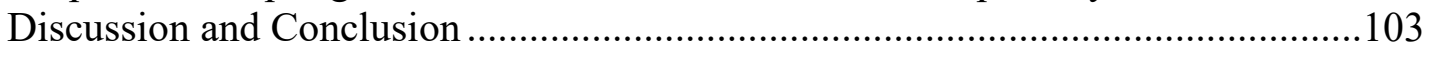

CHAPTER 6. SUMMARY AND CONCLUSIONS.....................................................108

LIST OF REFERENCES ......................................................................................................111

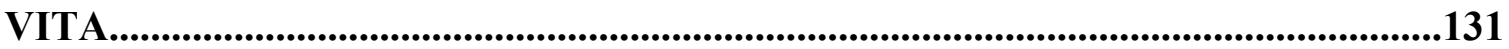




\section{LIST OF FIGURES}

Figure 1-1. Example of data appearance and illustration of data acquisition process.......4

Figure 1-2. Example LFP and spike data showing spike-LFP relationship and phaseamplitude coupling in primary visual cortex..................................................

Figure 1-3. Illustration of spike-LFP relationship during prominent LFP oscillation.....11

Figure 1-4. Illustrated demonstration of the communication through coherence hypothesis.

Figure 1-5. Illustration of effective communication through coherence with middle region integrating input from top and bottom regions at two distinct frequencies.

Figure 2-1. Example illustration of phase locking of spikes in the V1 and V4 LFP during gamma oscillation, and phase synchronization of gamma oscillations in V1 and V4.

Figure 2-2. Illustration of $\mathrm{r}$ value determination from a set of angular samples.

Figure 2-3. Example biased null phase distribution and phase normalization process. ..25

Figure 2-4. Illustration of test procedure for binned $r$ test to detect synchronization change in response to stimuli.

Figure 2-5. Evaluation of sensitivity and accuracy for the 2-sample binned $r$ test using randomization of real data.

Figure 2-6. Illustrated example outcomes for different parametric synchronization tests in different conditions.

Figure 2-7. Visualization of analysis steps involved in calculating the spike synchrony function.

Figure 2-8. Example result of frequency-resolved cross correlation of V1 LFP oscillations with LGN-V1 spike synchrony.

Figure 2-9. Conceptual illustration of the data analysis applied to determine the correlation between phase differences between mPFC-CA1 oscillatory LFP activity and PC simple spike activity in LS.

Figure 2-10. Illustration of Moore Rayleigh analysis of Respiration-LFP phaseamplitude coupling. .40

Figure 3-1. Visual-evoked narrowband and broadband high gamma oscillations in the visual system. 
Figure 3-2. Amplitude of high gamma oscillations is strongly linked to simultaneous thalamic high gamma activity.

Figure 3-3. Time-resolved estimation of spike synchrony is rhythmic in the high gamma range.

Figure 3-4. Amplitude increase of high gamma oscillations in V1 LFP occurs after synchronization of spike activity in the same frequency range.

Figure 3-5. Rhythmic synchronization of spike activity in dLGN and V1 is strongly linked to both narrow and broadband high gamma in V1.

Figure 4-1. Composite illustration of raw LFP signals and single unit PC spike activity recorded in the $\mathrm{mPFC}, \mathrm{CA} 1$ and cerebellar lobulus simplex.

Figure 4-2. Example analysis for one PC showing how phase differences across the full frequency spectrum of LFP oscillations $(0-100 \mathrm{~Hz})$ are represented in this cell's simple spike activity.

Figure 4-3. Summary of group results across all analyzed Purkinje Cells recorded in LS $(n=32)$ and Crus I $(n=17)$.

Figure 5-1. Respiration-locked oscillations and spikes throughout the olfactory bulb and neocortex.

Figure 5-2. Surgical placement of thermistor probe.

Figure 5-3. Comparison of respiration monitoring with minimally invasive and noninvasive thermistors in head-fixed mice. . .78

Figure 5-4. Plethysmographic recording of respiration during free movement. . .79

Figure 5-5. Measurement of sniffing behavior during odor presentation.

Figure 5-6. Disruption of olfactory bulb respiration oscillations with administration of inhibitory DREADDs channel activator Clozapine-N-oxide (CNO). .....85

Figure 5-7. Respiration-locked slow oscillations in the visual cortex and their dependence on OB activity.

Figure 5-8. Respiration-locked slow oscillations and spike activity in the primary sensory cortex for the trunk/abdomen (S1Tr). ............................................. 88

Figure 5-9. Respiration-locked activity in the LGN.

Figure 5-10. Respiration-locked slow oscillations in the basal forebrain (BF) before and after olfactory bulb inhibition by CNO. 
Figure 5-11. Effects of $\mathrm{CNO}$ and olfactory bulb inhibition on thalamocortical phase synchronization and visual perception.

Figure 5-12. Recording sites and raw data examples of simultaneous recordings of local field potentials (LFPs) in the awake mouse.

Figure 5-13. Hippocampal sharp-wave ripple (SWR) activity during the respiratory cycle in mice

Figure 5-14. Changes in respiration and local field potential (LFP) recorded in hippocampal CA1 region following Clozapine $\mathrm{N}$-oxide (CNO) injection in DREADD-mice $(\mathrm{n}=4)$.

Figure 5-15. Changes in hippocampal sharp-wave ripple (SWR) activity before and after Clozapine $\mathrm{N}$-oxide (CNO) Administration in mice with bilateral injections of DREADD vector in OB.

Figure 5-16. Slow respiration-locked cortical oscillations in the human brain, as measured by surface platinum electrode grid.

Figure 5-17. Respiration PAC statistical analysis for recording grids in 3 patients, and corresponding grid locations for significant gamma PAC. 


\section{LIST OF ABBREVIATIONS}

$\begin{array}{ll}\text { 2H } & \text { 2-Heptanone } \\ \text { Ac } & \text { Acetophenone } \\ \text { ACh } & \text { Acetylcholine } \\ \text { BF } & \text { Basal Forebrain } \\ \text { Bz } & \text { Benzaldehyde } \\ \text { CA1 } & \text { Cornu Ammonis Area 1 } \\ \text { CFC } & \text { Cross Frequency Coupling } \\ \text { Cit } & \text { Citronellyl Isobutyrate } \\ \text { CNO } & \text { Clozapine-N-Oxide } \\ \text { CTC } & \text { Communication through Coherence } \\ \text { dLGN } & \text { Dorsal Lateral Geniculate Nucleus } \\ \text { DREADDs } & \text { Designer Receptors Exclusively Activated by Designer Drugs } \\ \text { E4 } & \text { Ethyl Butyrate } \\ \text { E5 } & \text { Ethyl Valerate } \\ \text { E6 } & \text { Ethyl Hexanone } \\ \text { EEG } & \text { Electroencephalography } \\ \text { ECoG } & \text { Electrocorticography } \\ \text { ET } & \text { Ethyl Tiglate } \\ \text { LFP } & \text { Local Field Potential } \\ \text { LGN } & \text { Lateral Geniculate Nucleus } \\ \text { LS } & \text { Lobulus Simplex } \\ \text { mPFC } & \text { Medial Prefrontal Cortex } \\ \text { MUA } & \text { Multi Unit Activity } \\ \text { OB } & \text { Olfactory Bulb } \\ \text { PAC } & \text { Phase Amplitude Coupling } \\ \text { PC } & \text { Purkinje Cell } \\ \text { PLV } & \text { Phase Locking Value } \\ \text { PPC } & \text { Pairwise Phase Consistency } \\ \text { S1Tr } & \text { Primary Somatosensory Cortex for the Trunk Area } \\ \text { SWM } & \text { Spatial Working Memory } \\ \text { SWR } & \text { Sharp Wave Ripple } \\ \text { TRN } & \text { Thalamic Reticular Nucleus } \\ \text { V1 } & \text { Primary Visual Cortex } \\ & \end{array}$




\section{CHAPTER 1. INTERPRETATION AND SIGNIFICANCE OF ELECTROPHYSIOLOGICAL RECORDINGS}

\section{Purpose}

It takes a great number of neurons to accomplish even the simplest of tasks by even the smallest of mammalian brains. With each neuron's contribution to the task comes a small disturbance in the surrounding electric field, leading to the emergence of complex patterns of ionic currents as large populations are engaged in parallel. By recording these currents in a living brain, it is possible to identify consistent patterns and determine their correspondence with sensory input and behavior. Hopefully then, by understanding the basis of these patterns in depth, one can begin to link patterns of brain activity to more abstract functions, such as the representation and storage of information.

This chapter will begin with a brief history of neuronal synchronization and rhythmicity as a focus of study. It will then shift focus to the basic characteristics of electrophysiological data, and build towards explaining our current understanding of the role of coherent network oscillations in brain function. This will include both a description of experimental results as well as their place in a theoretical framework known as the communication through coherence hypothesis. The implications of this hypothesis will be described in detail and will help provide justification for analytic methods and experiments described in later chapters.

\section{Historical Context}

The means by which discrete entities like neurons collaborate to represent unified perceptions and initiate directed actions have long perplexed scientists and philosophers. Selective neuronal communication, determined by changes to synaptic strength and correlations among action potentials, was proposed by von der Malsberg in 1981 to serve the purpose of allowing neurons to build sensory representations from afferent impulses as they ascend in the brain's heirarachy ${ }^{1}$. He argued that networks with a topographical correspondence to stimulus features would necessarily generate temporally correlated activity when appropriate features were present, and silence or asynchrony when they were not. Although similar to earlier Hebbian theory of learning ${ }^{2}$, von der Malsberg challenged the notion of a cell assembly as an essential construct for encoding, due to its inflexibility.

Early evidence of von der Malsburg's proposal arrived in a pair of landmark studies by Gray and Singer in $1989^{3,4}$, which further characterized visual cortical response properties identified by Hubel and Wiesel ${ }^{5,6}$. In these studies Gray and Singer presented visual stimuli to an anesthetized cat while recording electrical responses from visual cortical tissue. They observed that neurons which exhibited a preference (increased firing rate) for the orientation and movement direction of lines in the visual field synchronized their spike activity ${ }^{3}$. Hubel and Wiesel previously showed that neurons with 
similar stimulus preferences were gathered within cortical columns ${ }^{5}$, and Gray and Singer further noticed that these columns exhibited high frequency (approximately $40 \mathrm{~Hz}$ ) electric field oscillations when the neurons within them were excited by their preferred stimulus $^{4}$. Furthermore, these synchronized spikes generated by common stimulus features aligned with the troughs of these oscillations, leading to the hypothesis that extrinsic oscillations - thought from an early stage to be driven by mutual excitation and recurrent inhibition ${ }^{7,8}$ - might play a role in the temporal organization of neuronal activity necessary for visual processing.

Today, these are commonly known as gamma frequency $(30-100 \mathrm{~Hz})$ oscillations, and mounting evidence supports the hypothesis that these and other frequencies of local field potential (LFP) oscillations reflect important mechanisms for temporal coding. Analysis of the LFP oscillations in the frequency domain became more commonplace as methods of spectral transformation were introduced to the field by Walter J. Freeman ${ }^{9,10}$ in studies where he described frequency shifts in olfactory bulb oscillations corresponding to changes in behavior. This data transformation allowed neuroscientists to apply an assortment of analytic tools that were originally developed in physics - to describe the behavior of waves and oscillators - for use in the quantification of neuronal oscillations and their relationship to synchronous spiking, and behavior. These methods of analysis became increasingly popular as computers became more powerful and affordable, and software implementation became more accessible.

Since the early 2000s, theories have solidified around the functional role of extrinsic oscillations in sensory processing ${ }^{11}$, multi-modal sensory integration ${ }^{12}$, and predictive coding ${ }^{13}$. One of the most influential of these theories was articulated by Pascal Fries (a former student of Singer at the Max Planck institute), and is known as the communication through coherence hypothesis ${ }^{14}$. This initial theory, first published in 2005 , argued that neuronal computation was critically dependent upon the synchronization of LFP oscillations between brain regions due to the fact that neurons were only sufficiently responsive to input during certain phases of these oscillations ${ }^{15}$. Fries reasserted this theory in a second perspective paper a decade later ${ }^{16}$, in which he took a stronger stance: that oscillations define neuronal representation of information in the brain, since they critically shape neuronal communication within characteristically hierarchical mammalian sensory systems. Furthermore, he and others have produced experimental evidence that a single brain region can maintain oscillatory synchrony with multiple other regions simultaneously at distinct frequency ranges ${ }^{17-19}$, or integrate oscillations from multiple frequencies to impose top-down order on bottom-up information flow ${ }^{20}$, thus providing insight that oscillations could play an important functional role in the integration of information from multiple sources. This theory will serve as the launching point for the current work. From here, I will describe the basic features of electrophysiological data, and how they relate to important mechanisms for brain function. 


\section{The Microelectrode Recording}

In the current work, all animal data were acquired with high-impedance microelectrodes. During an experiment, the voltage of the brain tissue at the tip of the electrode is measured with reference to the metal guide cannula directly above the dura, which is electrically coupled to the brain via saline. The voltage fluctuations captured by the headstage amplifier reflect currents generated by the synaptic input to the surrounding area as well as the action potentials occurring in neurons directly adjacent to the electrode tip. These two signals are initially superposed, but have different frequency profiles and can be largely separated by digital or hardware filters. The lower frequency range, typically $0.1-200 \mathrm{~Hz}$, represents the patterns of synaptic input, and is referred to as the local field potential (LFP) after this filtering process. Action potentials have a much higher frequency profile, and can be visualized as "spikes" by filtering the raw signal to within $200-10,000 \mathrm{~Hz}$ range. (For an illustration, see Figure 1-1.)

In the following experiments, the raw voltage signal undergoes initial amplification at the headstage, and is amplified a second time by a pair of hardware filter amplifiers before digitization. For digitization, LFPs and spikes are sampled at 2,000 and $20,000 \mathrm{~Hz}$, respectively.

\section{Determining Spike Times}

Whether information is reflected in timing or rate, the action potential is considered an irreducible unit of the neuronal code ${ }^{21}$, so capturing them reliably and estimating the time(s) of their occurrence accurately is of great value for many research questions. Unfortunately, a "spike" is not directly equivalent to a neuronal action potential, but it is more of an estimation that an action potential occurred based on patterns of high-frequency activity observed at the electrode. During an action potential, currents are induced to flow in the extracellular space by ions rapidly crossing the neuronal membrane. These secondary currents, which are perturbed by all other local currents $^{22}$, are what is detected by the electrode ${ }^{23}$.

"Spike sorting" is the process used to estimate the timing of action potentials, which is accomplished by algorithmically identifying events within the record that conform to a template or criteria specified by the investigator. This is an imperfect process, and controlled studies have revealed that spike sorting error is routinely above $10 \%$ for both false positives and false negatives ${ }^{24}$. Furthermore, discrepancies between spikes that were manually sorted by different users has been measured in the range of 20$30 \%{ }^{25}$. In order to have reasonable confidence that spikes correspond to action potentials, it is essential to acquire data with a high signal to noise ratio; preferably at least 4:1. 


\section{Raw Signal}

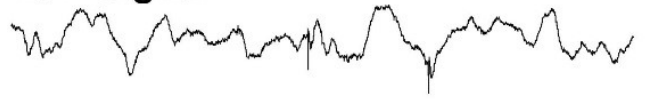

Local Field Potential

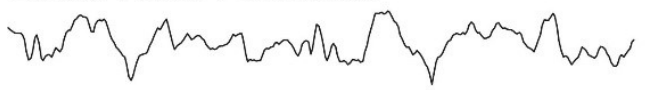

Spike
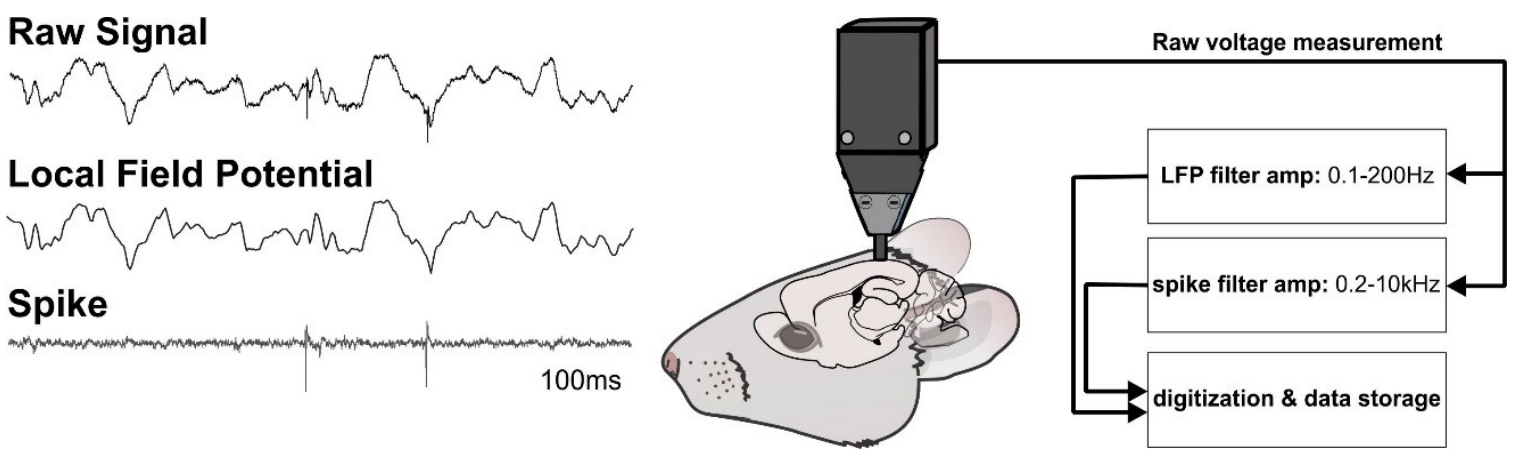

Figure 1-1. Example of data appearance and illustration of data acquisition process.

The voltage measured at the microelectrode tip relative to the guide cannula on the dura is amplified by the headstage. This is the raw signal, which is passed through a set of filter amplifiers in order to separate and amplify different frequencies which make up the local field potential and spike traces. After amplification, the LFP and spike signals are sampled at $2 \mathrm{kHz}$ and $20 \mathrm{kHz}$ respectively for digitization and storage. 


\section{Pre-processing the Local Field Potential (LFP) Record}

All pre-processing protocols should include a method to eliminate electrical noise at $60 \mathrm{~Hz}$. This is best accomplished using an algorithmic subtraction rather than applying a digital filter, since some essential LFP oscillations occur at $60 \mathrm{~Hz}$ intervals. The algorithmic method requires simultaneous recording of the ambient electrical noise during data acquisition.

In some special cases, further pre-processing is necessary to prepare LFP data for analysis, but for our purposes the signal is fit for post-processing and analysis after noise subtraction.

\section{Significance of LFP Oscillations}

What drives a neuron to fire? How do neurons communicate? How do neurons represent information? These are related but disparate questions that can be explored to some degree by studying the temporal relationship between oscillations in the LFP and spikes in various contexts. Each of the studies within this dissertation involve the interpretation of neocortical LFPs, therefore neocortical oscillations will serve as the primary focus of this introduction.

The obvious answer to what drives a neuron to fire is excitatory input, but there are also many factors that will drive a neuron to fire in response to one input but not another when virtually the same amount of excitatory current or neurotransmitter is delivered. Broadly speaking, preferential response to an input depends on the concurrent patterns of activity that are occurring in the target neuron's "local network"; patterns that are discernible by observing the LFP.

The LFP largely reflects extracellular current that is induced by transmembrane ion exchange at many synapses simultaneously, and can be interpreted as the aggregate output of many neurons onto the recording area $^{22}$. Due to the relative abundance of shortrange projections, the LFP is primarily a reflection of local neuronal activation, and is sometimes referred to as the population activity of the local network. Upswings and downswings in the LFP signal thus indicate whether synaptic activity surrounding the recording site is predominantly inhibitory or excitatory in its effect. Over time, patterns in the LFP can range from apparently chaotic with no appreciable interval or rhythm, to nearly sinusoidal. The more consistent oscillatory patterns are of particular interest, because they tend to reflect some stimulus property and/or cellular or network level resonance that promotes rhythmicity of neuronal activity, which in turn supports communication between rhythmically synchronized neurons.

Synchrony of excitatory input has long been emphasized as a critical factor in driving action potentials in post-synaptic neurons, because the effect of two synchronous excitatory inputs is typically greater than the sum of each input individually ${ }^{26}$. This supralinear effect is thought to be a consequence of several factors, including integration 
by active dendrites ${ }^{27}$, changes in the available pool of inactive voltage-gated channels that allow the inward current required for the initiation of an action potential ${ }^{28,29}$, and decreased membrane input resistance when excitation is prolonged ${ }^{30}$, resulting in smaller voltage changes for the same amount of trans-membrane current. Together, these factors cause neurons to favor rapid-onset excitatory currents with a sufficient slope. These factors also impart a preference for inputs to be delivered with sufficient frequency in the case of rhythmic synchronization ${ }^{31}$.

Furthermore, network inhibition allows excitatory input to take advantage of supralinear summation in three important ways. First, inhibition promotes synchrony by creating shared moments of neuronal silence that local neurons may recover from on similar timescales ${ }^{32}$. Second, inhibition allows inactivated ion channels to become deinactivated. Prolonged membrane depolarization can lead to an overabundance of voltage-gated channels becoming inactivated, making it more difficult or impossible for a neuron to reach action potential threshold. Inhibition can briefly hyperpolarize the membrane, thus allowing these voltage-gated channels to become available for activation again. Third, inhibition coupled with excitation creates rhythmic oscillatory activity in the network, which can modulate firing rates so that they match the frequency preferences of target neurons. Conversely, the frequency preferences of neurons can also stabilize network oscillations at a consistent frequency ${ }^{33}$. Together these intrinsic and extrinsic factors cause a greater degree of synchrony among adjacent neurons, increase the prominence of rhythmic activity within a local network, and bias oscillations to occur at mutually preferential frequencies for sets of interacting neurons. Critically, this results in neuronal communication that is selectively enhanced when it occurs rhythmically in a frequency and phase-aligned manner.

\section{The Spectrum of LFP Oscillations}

LFP oscillations in different frequency ranges are generated by distinct mechanisms, but all are associated with neuronal synchrony, and either drive local units to spike at a specific phase (Figure 1-2) or result from the intrinsic properties of neuronal populations that direct output to other areas.

Here I will briefly discuss the frequency bands in which neuronal oscillations occur, in the terms of their conventional nomenclature. This includes delta $(0.5$ to $4 \mathrm{~Hz})$, theta (4 to $8 \mathrm{~Hz}$ ), alpha ( 8 to $12 \mathrm{~Hz}$ ), beta (12 to $30 \mathrm{~Hz}$ ), and gamma oscillations (30 to $100 \mathrm{~Hz}$ ). This will include information about the locations where these oscillations commonly originate or are most prominent, what events trigger them, what is known about their underlying physiology, and what is currently understood about their functional role in the brain. 

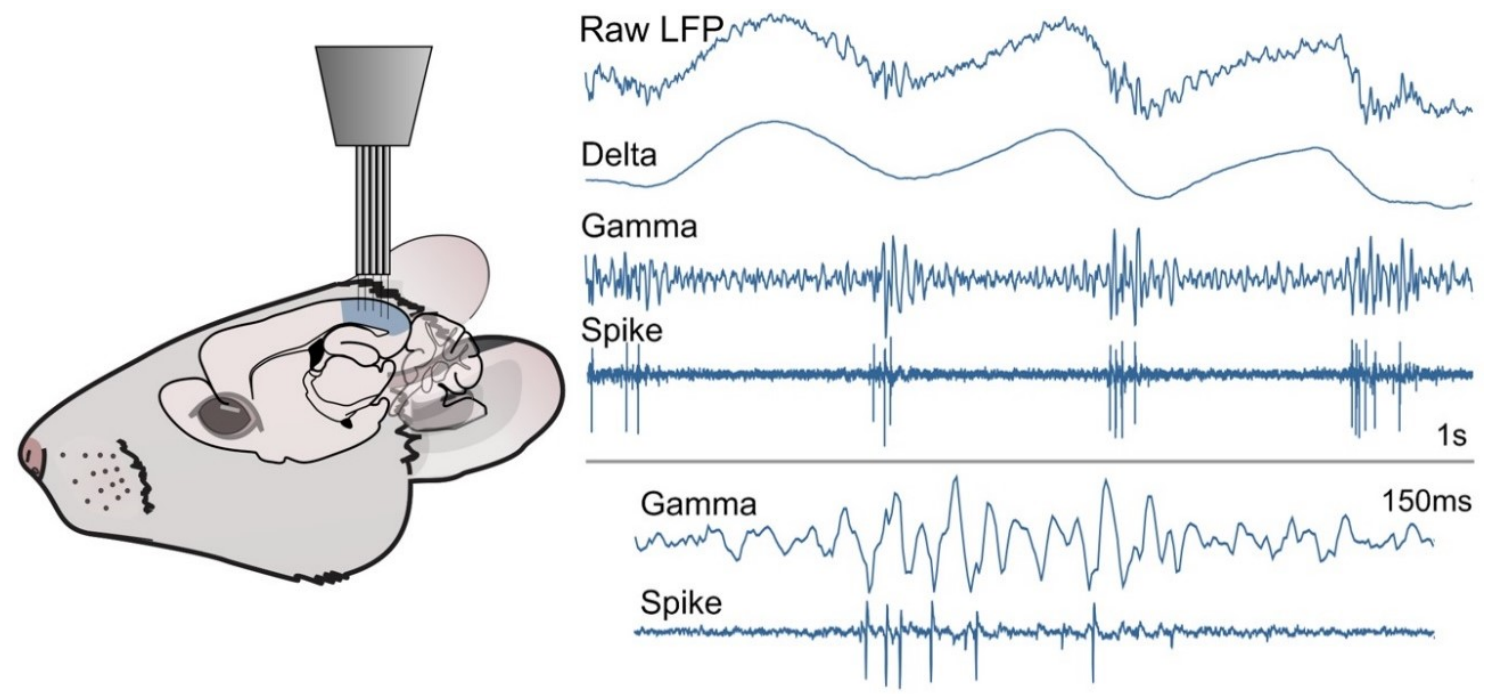

Figure 1-2. Example LFP and spike data showing spike-LFP relationship and phase-amplitude coupling in primary visual cortex.

The LFP at the top shows prominent oscillations in two separate frequency ranges: delta $(1-4 \mathrm{~Hz})$ and gamma $(>30 \mathrm{~Hz})$. With respect to the delta oscillation, high amplitude bursts of gamma oscillations and increased spike activity can be seen nested into the trough phase of delta. With respect to gamma, spiking of the isolated unit occurs predominantly at the trough of the local gamma oscillation. The relationship is not absolute; note the second spike which occurs at the gamma peak, and several oscillation troughs with no observed spike activity. 


\section{Delta Oscillations}

Delta oscillations are slow oscillations in the range of $0.5-4 \mathrm{~Hz}$ that are observed with high amplitude in human scalp electroencephalography (EEG) during the deeper stages of sleep ${ }^{34}$. Oscillations in the delta range are also seen to a lesser extent during the awake state as a reflection of slowly evolving trends in the activation of pyramidal neurons across broad cortical regions ${ }^{35}$, but can be emblematic of cortical pathology if excessive in amplitude or if amplitude is not proportional between cerebral hemispheres ${ }^{36}$.

During sleep, delta oscillations are most prominent in layer $2 / 3$ and layer 5 , with opposite polarity $^{37}$. These oscillations emerge in the cortex due to spontaneous patterns in pyramidal neuronal populations ${ }^{37}$, but can also be influenced by thalamic input ${ }^{38}$. In thalamic neurons, delta rhythmicity can be intrinsically generated by the interaction of opposing currents; the hyperpolarization-activated cation current $\left(\mathrm{I}_{\mathrm{h}}\right)$, and the transient low-threshold calcium current $\left(\mathrm{I}_{\mathrm{T}}\right)^{39}$. These intrinsic oscillations are initiated by hyperpolarization that is induced by input from inhibitory neurons in the thalamic reticular nucleus (TRN), which are excited by corticothalamic collaterals from layer $6^{39}$.

These slow oscillations appear to be an essential feature of long-term memory storage that occurs during sleep. Broad activation of cortical, thalamic, and hippocampal neurons is thought to initiate sequences of neuronal activity that ultimately strengthen connections between and within these regions ${ }^{40}$. Delta oscillations are aligned with thalamocortical "spindle" oscillations in the 7-15 Hz range, which are in turn aligned with hippocampal "ripple" oscillations occurring upwards of $100 \mathrm{~Hz}$, resulting in neuronal interactions between thalamus, cortex, and hippocampus that are temporally precise at high frequency ${ }^{41}$. Since fast rhythmic synchrony is thought to induce synaptic long term potentiation, delta oscillations are thought to be an initiating event for the synaptic changes that occur during memory consolidation in sleep ${ }^{40}$.

\section{Theta Oscillations}

Theta oscillation are relatively slow oscillations within the 4-8 $\mathrm{Hz}$ frequency range in scalp EEG, or $4-12 \mathrm{~Hz}$ in LFP recordings of the hippocampus. The theta oscillation in the hippocampus is widely studied for its role in memory function, and is the most persistent and sinusoidal rhythm in the entire brain ${ }^{38}$. This oscillation becomes higher in amplitude with voluntary movement, as well as higher in frequency during locomotion $^{38}$. Hippocampal theta is dependent on theta-rhythmic input from long-range inhibitory neurons of the medial septum-diagonal band of $\mathrm{Broca}^{42}$, which synapse on inhibitory neurons to disinhibit primary neurons of the hippocampal complex in a rhythmic fashion ${ }^{38}$. Septal neurons oscillate intrinsically at a theta frequency, but also receive excitatory feedback from hippocampal neurons, making the theta reverberation more robust and adaptable ${ }^{38}$. Hippocampal theta is thought to allow the hippocampus to separate the functions of memory encoding and retrieval in time ${ }^{43}$ by selectively 
synchronizing hippocampal activity with activity in different regions so that interference is minimized ${ }^{17}$.

Theta in the neocortex can be a reflection of interaction with the hippocampus ${ }^{38,43}$, although it is more commonly studied for its influence on higherfrequency cortical oscillations ${ }^{44}$, and how those influence the precise timing of spikes thought to carry information from one cortical region to another ${ }^{45,46}$. This theta is generated by the same extrinsic properties that give rise to cortical delta rhythms ${ }^{35}$, and appears to not only influence the amplitude of higher-frequency oscillations, but also timing of "phase transitions" between desynchronized and synchronized network states, which are thought to represent the receiving and transmission of information, respectively ${ }^{47}$. This will be covered in more detail in the next section.

\section{Alpha Oscillations}

Alpha oscillations are those in the $8-12 \mathrm{~Hz}$ frequency range, and are most commonly observed in EEG of the occipital cortex during wakefulness when the eyes are closed. In fact, this oscillation was the first to be identified, and was referred to as Berger's wave, after Hans Berger who discovered it in the 1920s.

The alpha rhythm is generated by reverberations in the thalamocortical circuit while no sensory information is being transmitted ${ }^{37}$, and thus is thought to constitute an "idle" mode for the network. It shares an origin with the "spindle" rhythm that occurs during sleep, although the latter is thought to have a different function. Both oscillations appear to be entrained by rhythmic thalamic inhibition delivered by the thalamic reticular nucleus (TRN). The TRN receives collateral input from both corticothalamic as well as thalamocortical projections, and the latter are necessary for generation of the rhythm ${ }^{37}$. Thus, synchronous alpha-frequency rhythms arise in thalamus and cortex due to rhythmic interaction between thalamic and TRN neurons, where thalamic neurons excite TRN, leading to transient inhibition in thalamic neurons that is followed by a synchronous rebound $^{37}$. In the case of spindle oscillations, the synchronous rebound of thalamic activity is synchronized with high frequency ripple activity in the hippocampus ${ }^{41}$, which together are thought to modulate synaptic connections for memory consolidation ${ }^{40}$.

\section{Beta Oscillations}

Beta oscillations occur in the frequency range of 12-30 Hz. In human EEG, beta oscillations are observed on the scalp above sensory and motor cortices during the execution of movement ${ }^{48}$. These oscillations are a result of several factors, including rhythmic inhibition ${ }^{48}$, synchronous rebound of excitation in layer 5 pyramidal cells coupled via gap junctions ${ }^{49}$, and the intrinsic preferences of these layer 5 pyramidal neurons ${ }^{50}$. With regard to this movement-related oscillation, the rhythmicity of neuronal activity is determined by cortical mechanisms, while the strength of excitation and oscillations amplitude are maintained by a sensorimotor loop, with corticospinal neurons initiating movement, and muscles providing proprioceptive sensory feedback to the 
cortex $^{48}$. High amplitude beta oscillations have been identified as a biomarker in Parkinson's disease, which is consistent with the hypothesis that sensorimotor beta is related to the computation of the current tasks being executed ${ }^{51}$; if this command becomes inflexible, it becomes difficult to initiate or alter intended movement.

Sustained beta oscillations are observed in the olfactory system, and appear to be functionally related to odor-related learning and habituation, although the mechanisms that generate these rhythms and their specific functions are still poorly understood ${ }^{52}$. Electrical transients with the shape of a single beta oscillation are more widely observed in the neocortex. These are most commonly studied in the monkey visual system, where they appear to provide transient inhibition to pyramidal neurons during saccades ${ }^{53}$, and modulate higher frequency rhythms that carry information from lower to higher visual

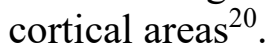

\section{Gamma Oscillations}

High frequency oscillations, conventionally known as gamma oscillations (30-100 $\mathrm{Hz}$ ), result from the cyclic alternation between predominantly excitatory and predominantly inhibitory population activity in a local network ${ }^{54}$. This oscillation is perhaps the most studied in neuroscience, as it arises related to attention ${ }^{55}$, sensory processing $^{3}$, movement ${ }^{56}$, decision making ${ }^{57}$, memory storage and retrieval ${ }^{17,58}$, and spatial navigation $^{59,60}$. It is thought to constitute a fundamental mechanism of neuronal computation, as it allow neurons to communicate selectively on the basis of signal timing relative to the phase of the network oscillation ${ }^{61-63}$. Gamma oscillations are initiated by excitatory afferent impulses to the neocortex activating GABAergic neurons, which have brief inhibitory and/or shunting effects on their local post-synaptic targets ${ }^{64}$. After GABA is cleared, there is a rebound in excitatory activity which reignites the cycle ${ }^{64,65}$. Overall, the oscillation in the network drives sub- and super-threshold membrane voltage oscillations in individual neurons, which affects their responsiveness to afferent synaptic input $^{63,66}$. The link between cyclic activity patterns in the network and input gain in individual neurons again leads to a broadly consistent relationship between the phase of these LFP oscillations and the occurrence of spikes (Figure 1-3).

The precise spike-phase relationship within the gamma cycle is also thought to provide information value ${ }^{61}$, although experimental evidence of this is not conclusive ${ }^{67-69}$. The basis for this hypothesis is that the phase lag of post-synaptic spikes should be inversely proportional to the strength of the input, given the known characteristics of synaptic connections in the neocortex ${ }^{61}$. Cortical neurons establish synaptic connections in such a way that proportional populations of excitatory and inhibitory neurons are active at any given time ${ }^{70,71}$, and each neuron receives excitatory and inhibitory synaptic input with comparable strength ${ }^{72-74}$. This balance of excitation and inhibition in the network is known to determine the amplitude and frequency of gamma oscillations ${ }^{75}$, as well as provide conditions where neurons have a roughly equal barrier of inhibition to overcome in order to respond to an excitatory input with an action potential. Given these conditions, the neurons within a region that receive the strongest input will most 

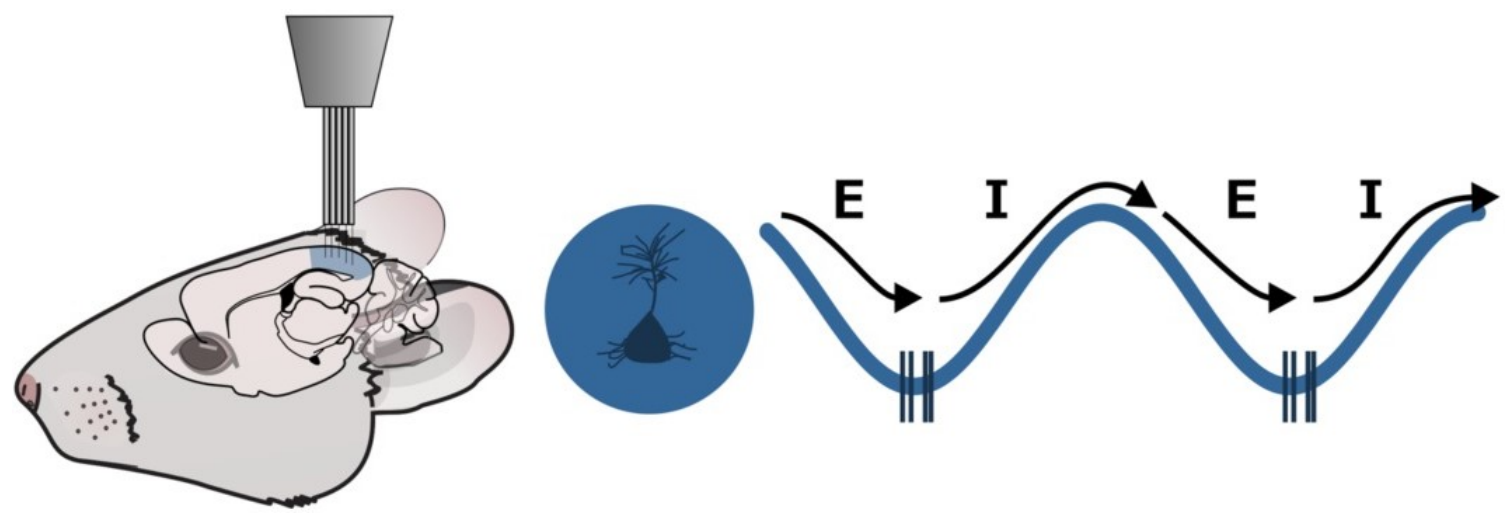

Figure 1-3. Illustration of spike-LFP relationship during prominent LFP oscillation.

Lighter waveform depicts LFP within the area of the recorded unit, and dark lines depict the occurrence of spikes. Oscillations in the LFP correspond to cyclic alternation between predominantly excitatory and predominantly inhibitory population activity in a local network. As a result, the neuron receives synaptic input during corresponding phases of the LFP oscillation, increasing the likelihood of spontaneous or evoked spike activity during the excitatory phase. 
effectively overcome network inhibition and spike the earliest within the oscillation ${ }^{61}$. This selectivity is likely enhanced by the rapid silencing of weakly-excited neighboring neurons via recurrent inhibition. Altogether this scheme allows for spike-gamma relationships in the target region to encode information about the strength of the input it received, which is determined by some potentially meaningful combination of the spike rate, the number of converging inputs, and the synchrony of converging input from the source region(s) ${ }^{76}$. This proposed mechanism of information transmission is known as "phase coding."

\section{The Role of Oscillations in Cortical Information Processing}

\section{Coherent Oscillations and Inter-Neuronal Transmission}

So far I have described how neurons interact with their immediate neighbors, but how do neurons communicate effectively when they are located in different brain regions? This question can be approached as an extension of the previous, in that effective communication between brain areas takes advantage of those local network factors that make neurons preferentially responsive to excitatory input. This makes interareal neuronal communication a process of synchronizing the rhythms of local networks in a coherent manner, and allows communication between individual neurons in the regions to be both effective and selective, ultimately defining the neuronal representation of sensory information as it is transmitted from one brain region to another.

What is coherence? Coherence is a term developed in physics to describe coupled oscillators, but for our purposes in this section it describes a correlation in rhythmic neuronal activity that is both stable across time and limited to a specific frequency range. The next chapter will describe how the coherence metric can be mathematically defined, but the use of the term "coherence" in this chapter refers to the general phenomenon of oscillatory synchrony.

Coherence of rhythmic activity within the gamma range is thought to convey information in the bottom-up direction, as gamma oscillations in primary sensory cortices entrain gamma oscillations observed in higher-order sensory regions ${ }^{55}$. As will be discussed in detail in Chapter 3, coherent gamma oscillations also drive temporally aligned spike activity in some thalamic nuclei and the primary cortices they project to. This is the simplest form of communication through coherence (CTC), also referred to as bottom-up CTC or unidirectional CTC. In this scheme, the source region sends a rhythmic burst of excitatory input to the target region, exciting the local network so that it undergoes an oscillation at the same frequency ${ }^{54}$. This causes the target region to become preferentially receptive to input from the source region, since input necessarily arrives

during the excitatory phase of the emerging oscillation. Consequently, this also causes the target region to become less responsive to excitatory input that is not well aligned with its local oscillation, leading to a further increase in the fidelity of transmission from the source to the target (Figure 1-4). 

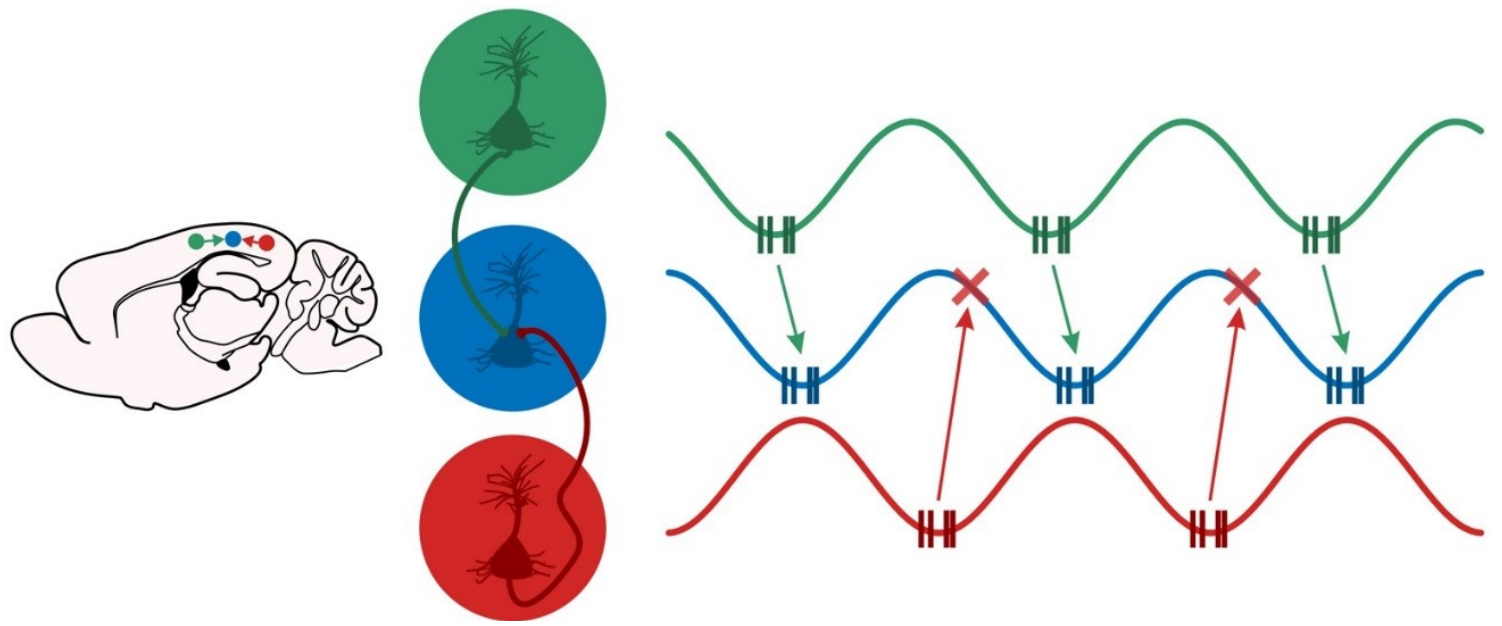

Figure 1-4. Illustrated demonstration of the communication through coherence hypothesis.

Lighter waveform depicts LFP within the area of the recorded unit, and dark lines depict the occurrence of spikes in respective neocortical areas. Red " $X$ " represents failure to elicit spike response. Imagining each of these units experiences the same relationship to the LFP as in Figure 1-3, the communication through coherence hypothesis describes how relative timing of LFP oscillations affects communication between the individual neurons. In this case, the top (green) and bottom (red) neurons are trying to excite the middle (blue) neuron. Each region is oscillating at the same frequency, but with a slightly different initial phase, leading to the green-blue pair having a different phase relationship than the red-blue pair. When the green and red neurons fire with respect to their LFPs, their spike output is received at different LFP phases by the blue neuron, resulting in differences in input gain. Therefore, all else being equal in terms of spike count and synaptic strength, the green neuron was more effective at eliciting a response in the blue neuron solely on the basis of their phase relationship. 
At first inspection, the establishment of bottom-up gamma coherence by two regions seems inevitable or even trivial based on what we've established, but the factors that determine which specific cortical regions become entrained are crucial for the processing of sensory information. For example, the specific subset of synchronized neurons in the source region determines which post-synaptic areas receive excitation, and the target regions with the greatest degree of synaptic overlap will receive the strongest drive to fire, and subsequently silence their neighbors with recurrent inhibition. This leads to a winner-take-all phenomenon that is similar to phase coding, but for the recruitment of cortical columns to exhibit rhythmic gamma activity ${ }^{69}$. The neurons within the activated column(s) then excite their targets and the process repeats itself, potentially generating a wave of gamma activity that propagates across a span of the cortex. Thus, changes to the activated neuronal subset within an early source region can affect the propagation path of a gamma wave, and all neuronal communication within it ${ }^{69}$. Interestingly, early anatomical analysis of the cortex predicted that excitatory input would propagate as a wave or avalanche ${ }^{77}$, and some of the earliest models for the cortical representation of information involved chains of neuronal sets being activated synchronously in specific sequence ${ }^{78,79}$, despite an incomplete understanding of what synchronizing mechanisms were at play $^{80}$.

Gamma-rhythmic activity in the cortex is rarely sustained for more than a few hundred milliseconds, and most commonly occurs in alternating "bursts" and "beats" that are driven to occur at a theta frequency ${ }^{47}$. These bursts represent moments when activity in the local network is relatively stable ${ }^{47}$, and a specified subset of pyramidal neurons are able to effectively transmit excitatory signals to their targets ${ }^{61}$. Thus these gamma bursts are thought to represent the "transmitting" phase of the cortical network ${ }^{47}$. By contrast, between these bursts are "beats" (sometimes referred to as "phase transitions"), or moments of relative instability in the local network marked by a rapid decline in the amplitude of gamma ${ }^{47,81}$. This is thought to constitute a "receiving" phase for the local network, when gamma activity is desynchronized between adjacent areas, such that oscillations can be easily entrained with rhythmic input. The significance of beats is apparent when the spatial arrangement of gamma oscillations is investigated from electrode grid recordings of the cortical surface. As gamma amplitude declines within a region, a spatial focus is able to emerge where the phase of gamma is substantially advanced with respect to surrounding areas ${ }^{47}$. The output from this focus becomes out of sync with its surroundings to the point where it delivers inhibition over the duration of excitatory rebound in other areas, leading to a nearly complete collapse in gamma rhythmicity ${ }^{47}$. As a consequence, the oscillation in the focal region becomes increasingly stable due to less inhibitory interference, and gains amplitude while entraining oscillations in surrounding areas ${ }^{47,81}$. The spatial focus that emerges during a beat is theoretically the area with the strongest input at the appropriate time of this receiving phase $^{47,81}$. Given the iterative nature of these oscillatory patterns, it is apparent that spatiotemporal arrangements of excitatory input are processed by gamma oscillations on at least two timescales, with the first being a single oscillation cycle, and the second being the span of time between bursts of multiple oscillation cycles. Thus, it may be more appropriate to refer to bursts as the "intra-areal processing phase" and beats as the "interareal processing phase", rather than "transmitting" and "receiving" phases, respectively. 
Top down influences, such as attention and expectation, also clearly play a role in determining the strength and path of inter-areal gamma coherence, although the mechanisms of action in this realm are not yet fully understood. The necessity for topdown influences in sensory processing is best illustrated by examples where a stimulus is relevant in one context and irrelevant in another, a common example being a red light at a traffic stop versus a red light in a movie theater. The input itself is invariant, but the two experiences generated by the input are very different. Similarly, sensory responses in early sensory brain areas are relatively consistent, yet the responses in higher-order cortices are variable with the context ${ }^{82}$. With the input being the same and the primary neuronal representation being the same, somehow the brain represents the two differently through modifications to neuronal communication.

One noteworthy experiment created a context like this in order to test whether changes in coherence played a role ${ }^{83}$. Two visual stimuli were presented to a monkey, who was trained to attend to one while ignoring the other. The experimenters acquired simultaneous recordings from two regions of the primary visual cortex (V1) corresponding to the visual location of the stimuli, and a mutual target region visual area 4 (V4). When the monkey performed the task, every region exhibited prominent gamma oscillations within the same approximate frequency range, but only the oscillations within the attended portion of V1 were coherent with V4. This suggests that some top-down influence allowed the neuronal group representing the attended stimulus in V1 to become preferentially synchronized with the neurons in V4, while the unattended stimulus failed to establish bottom-up coherence with its target.

It is still unclear what top-down mechanism is at work here, but it likely results in some potentiation of the appropriate primary sensory region corresponding to expected stimulus location. This is supported by experiments showing that merely the expectation of a sensory cue is sufficient to elicit synchronous cortical spiking ${ }^{84}$. In the case of the experiment probing visual attention, the coherent region of $\mathrm{V} 1$ exhibited a slightly higher gamma oscillation frequency ${ }^{83}$, likely leading to winner-take-all entrainment of V4 due to excitatory impulses arriving there sooner ${ }^{69}$. Top-down influences are also associated with beta oscillations that modulate the robustness of gamma ${ }^{19,20}$. This relationship between expectation, beta oscillations, and neuronal potentiation is also apparent following saccadic eye movements, immediately after which a beta wave is observed in V1, briefly increasing neuronal excitability ${ }^{53}$.

Lastly, bi-directional CTC is thought to be a mechanism for synchronizing columns of neurons within a sensory cortical region. This is considered a crucial factor in the recruitment of adjacent neurons for the representation of contiguous visual images ${ }^{85}$, in order to convey a synchronized and topographically meaningful message to higherorder regions ${ }^{86}$. Bi-directional CTC is best understood as mutual entrainment of two areas via simultaneous uni-directional CTC, although conduction delay creates a unique problem $^{87}$. In this case, vertical delays across the cortical layers matches lateral delays between regions, allowing new cycles of output to be synchronized with inputs from previous oscillatory cycles ${ }^{88}$. 


\section{Parallel Streams of Coherent Neuronal Communication}

What happens if one region requires distinct coherent communication with two other regions? Increasing evidence suggests that this is accomplished via the synchronization of oscillations at distinct frequencies, which are superposed in the LFP (Figure 1-5). This was first demonstrated in the hippocampus, where distinct frequencies of gamma oscillations were shown to support the incoming and outgoing streams of communication from $\mathrm{CA} 1^{17}$. Similar configurations of coherent oscillations have also been shown in the monkey visual system, with $\mathrm{V} 1$ receiving information from thalamus at a beta frequency $(\sim 20 \mathrm{~Hz})$ and transmitting it to higher cortical regions at a gamma frequency $(>30 \mathrm{~Hz})^{18}$. This mechanism is considered important to prevent ongoing processing from disrupting the input of sensory information that may be changing over time.

\section{Cross-Frequency Coupling}

As in the case of top-down beta discussed earlier, gamma oscillations are known to be modulated by concurrent lower-frequency oscillations in the network. This is referred to as cross-frequency coupling (CFC) or phase-amplitude coupling (PAC), and involves the modulation of gamma oscillation amplitude by the phase of a slower oscillation, typically theta ${ }^{89}(4-12 \mathrm{~Hz})$ or delta ${ }^{90}(1-4 \mathrm{~Hz})$. The mechanisms that underlie PAC are likely the same as those that generate network resonance at a gamma frequency in response to sufficiently strong excitatory input, although rhythmic input of neuromodulators may also play a role ${ }^{91}$. Graph theory models of cortical networks can generate PAC patterns without consideration for the effects of neuromodulators ${ }^{92}$.

The strength of the PAC relationship is correlated with performance in cognitive tasks in humans ${ }^{89}$, suggesting PAC mechanisms play an important role in cognitive processing. Gamma oscillations are critical for synchronizing neuronal activity on a small spatial scale relative to the vast amount of cortical tissue necessary for normal cognitive function, whereas slow oscillations reflect larger population patterns over broader areas ${ }^{22}$. Thus, it has been proposed that slow oscillations first work to loosely synchronize larger populations prior to the occurrence of gamma activity, thus increasing the frequency of coherent oscillations to increase the temporal precision of neuronal firing between engaged local networks ${ }^{12}$.

\section{Conclusion}

Together, can this evidence tell us anything about how neurons represent information? It is tempting to say that, to the extent that specific information could be represented by which groups of neurons are firing together, these groups are perhaps defined by the specificity or breadth of top-down projections, and the mechanisms they 


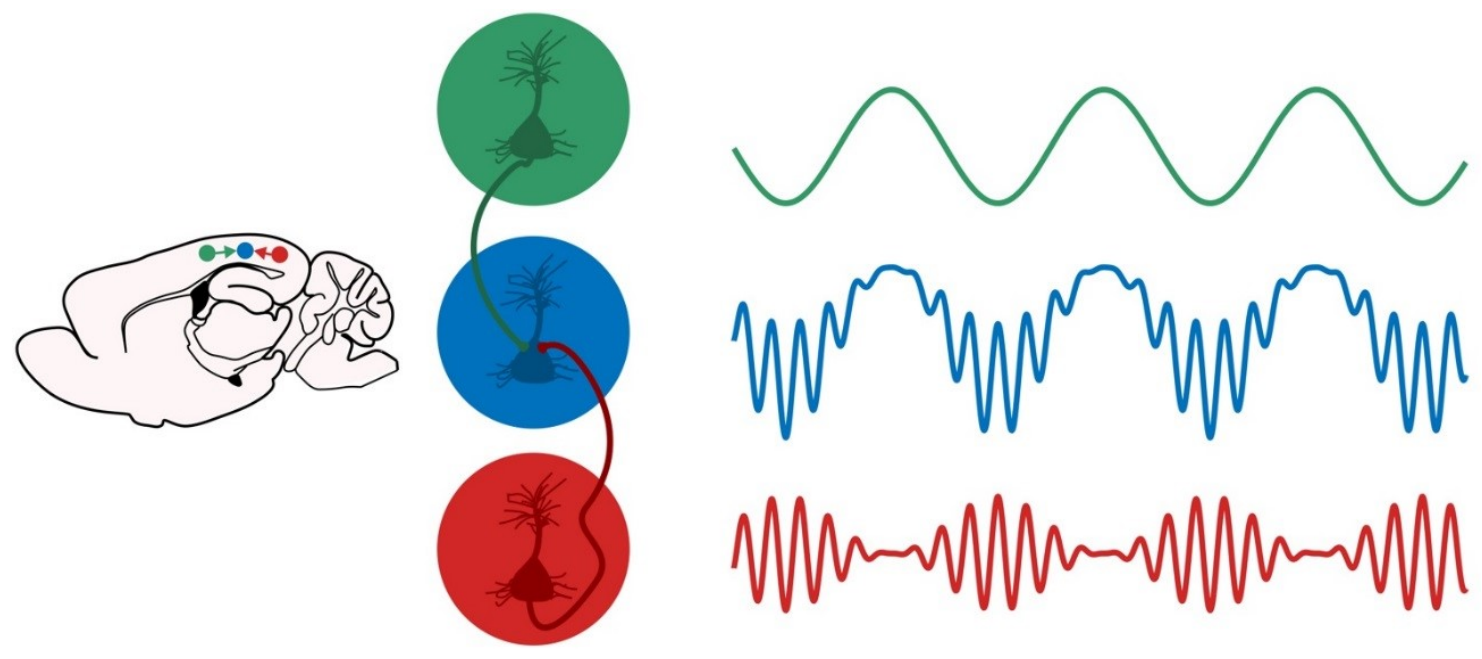

Figure 1-5. Illustration of effective communication through coherence with middle region integrating input from top and bottom regions at two distinct frequencies.

Colored lines represent LFP traces in corresponding regions. In this example, neurons within the top (green) and bottom (red) regions are trying to communicate effectively with neurons in the middle (blue) region. Assuming conduction delay is negligible, oscillations are occurring in a manner to support effective communication between both green-blue and red-blue paired regions, at a lower and higher frequency, respectively. 
utilize to promote CTC in competition with un-attended stimuli ${ }^{11}$. Thus meaningful perception may be created by the predictive coding that takes place in higher brain regions, and the bottom up transmission that serves to confirm or deny the prediction ${ }^{93,94}$. Although the neuroscience of this concept is fairly new, philosophically this is well aligned with the concept of intentionality; that it is not sufficiently meaningful for internal aspects of the brain to passively exemplify aspects of the environment, but the intention to perceive specific aspects of the environment is a fundamental prerequisite to perceive them. Of course, how the meaningful predictive code could develop from infancy is still unresolved. This concept is known to date back to at least the $13^{\text {th }}$ century ${ }^{95}$, and has influenced a series of important philosophical works about relationships between the brain, body, and mind.

Given this emphasis on top-down influences in perception, one would expect the cells involved in a meaningful group to be those in two or more regions which, when engaged at the proper time, allowed an afferent impulse to successfully traverse a rung in the hierarchy of the brain's structure ${ }^{96}$, whether it be from thalamus to cortex ${ }^{97,98}$, from

one cortical region to another ${ }^{99,100}$, or from one layer to another in a cortical column ${ }^{94}$. This may be one reason why the search for invariant cell assemblies within sensory regions has not been very fruitful, while stimulus-evoked coherence between regions is routinely observed.

\section{Overview of Following Chapters}

The focus of the remaining chapters will be on identifying oscillatory neuronal synchronization between different brain regions and in different contexts to better understand its role in brain function. The next chapter will provide an overview of the analytic tools used to identify and quantify this form of synchrony. Chapter 3 will present new evidence that coherent rhythms are necessary for visual thalamocortical transmission in the awake mouse, an important projection that has not been previously evaluated for coherent facilitation. Chapter 4 contains evidence of cerebellar activity as a possible modulator for the transient alignment of prefrontal and hippocampal rhythms, which have been implicated in various memory functions. Lastly, Chapter 5 will summarize a suite of findings related to the modulation of rhythmic brain activity by the respiratory cycle, including both rodents and humans. 


\title{
CHAPTER 2. DEVELOPMENT OF NOVEL METHODS FOR ASSESSING NEURONAL SYNCHRONY AND BRAIN FUNCTION THROUGH LFP AND SPIKE ANALYSIS
}

\begin{abstract}
Purpose
Thus far I have discussed the importance of neuronal synchrony for effective neuronal communication, and how this is reflected qualitatively in the electrophysiological record as temporally correlated oscillations and spikes. Now I will begin to cover the methods used to identify, quantify, and statistically evaluate these features, while keeping in mind the physiological impetus for doing so. This chapter will give a brief overview of analytic concepts, then describe the rationale and development process for the novel analytic methods used to arrive at the conclusions in later chapters.
\end{abstract}

\section{LFP Frequency, Phase and Amplitude Estimation}

The first step in virtually any analytic approach to LFP oscillations is to transform time-series data into the frequency domain. This accomplishes a few things simultaneously. First, it separates the single LFP time-series into distinct frequency components, which often reflect different physiological processes, as discussed earlier. Secondly, representing the evolution of neuronal activity as a set of oscillations creates numerical descriptors for the data that are possible to investigate separately, or combine and analyze in new ways. Specifically, oscillations are defined as having a frequency, amplitude, and phase at each moment in time, so estimating these values from the LFP time series can allow, for example, the estimation of a frequency-specific amplitude increase in response to a stimulus, or the quantification of the relationship between spike time and local oscillation phase.

This often involves some variation of a Fourier transform, or the representation of the complex LFP waveform as the unique sum of many sine waves with different frequencies, amplitudes, and initial phases. Unfortunately, this approach has several obstacles in its application to LFP analysis, all stemming from the fact that it imposes some assumptions on the data which are not fully met. The first such assumption is signal stationarity, or the assumption that the mean and variance of the data segment to be transformed are stable across time. This is not true with LFP data, since brain activity varies dramatically across different time periods. This problem is addressed by slicing a recording period into short time pieces within which signal variability aside from oscillatory activity is supposedly negligible, allowing for the implementation of the transform. Unfortunately, this accommodation reveals another important tradeoff that occurs in time-frequency analysis, which is that between resolution in the time domain (when did the oscillation occur?) and the frequency domain (what was the oscillation frequency?). One would ideally like to be able to estimate the exact phase, frequency, and amplitude of the prominent oscillations in the LFP for every sampled moment in time, but it is not possible to accomplish this by dividing the signal into infinitely short pieces. 
Currently, one of the most effective and intuitive approaches is to instead begin the analysis procedure with a band-limited portion of the LFP signal, and then use a modified transform known as the Hilbert transform, which returns a complex signal with easily discernable estimates of phase, amplitude, and frequency (within the specified bandwidth) for each time point ${ }^{101}$. This is accomplished by first applying a specific bandpass filter to the LFP recording to isolate the frequency range of interest. For technical background on the Hilbert transform and its application in electrophysiology, Freeman wrote an excellent guide ${ }^{102}$. Similar approaches involving a wavelet transform are equally effective ${ }^{103,104}$ but less intuitive, and will not be discussed in detail here.

\section{Oscillation Parameters in Context}

What do each of these parameter estimates represent in terms of brain function? To give a clear example, imagine there was interest in knowing whether gamma oscillations within the $40-50 \mathrm{~Hz}$ range are related to the processing of a visual stimulus, specifically in terms of synchronizing the local activity in V1 in order to relay an excitatory impulse to V4 (Figure 2-1). One can begin by filtering the LFP for resonance in the 40-50 Hz range, and implementing the Hilbert transform to create time-resolved estimates of amplitude and phase. Importantly, the gamma-frequency component represents only a portion of the overall activity, but hopefully this process of filtering will allow us to specifically probe the network mechanisms that underlie this oscillatory capacity of the network.

The amplitude of V1 and V4 gamma oscillations should always be the first parameter of interest, because if appreciable gamma oscillations never arise then it is reasonable to conclude they do not play a functional role. As mentioned in the previous chapter, gamma-rhythmic postsynaptic transmembrane current serves to excite neurons with precise synchrony, and as a consequence generate the extracellular field oscillations observed in the LFP. Thus, one could first look at amplitude values in the filtered range following the stimulus in order to determine whether the mechanisms that underlie gamma oscillations in the local network have been engaged by the stimulus.

Secondly, the phase progression of gamma oscillations offers insight into network interactions that can be difficult to derive from measures in the time domain. Realistic phase estimates require that oscillation amplitudes rise above the level of measurement noise. The instantaneous phase of these gamma oscillations, which is given as an angular value between 0 and $2 \pi$ for each time sample, can serve as an indicator for whether excitatory or inhibitory currents are predominating the local network. This can be further supported by investigating the set of phase values that correspond to local spike times; if an oscillation detected in the LFP drives synchronous activity, the phase values corresponding to local spike times will predominantly point to the phase of excitation (Figure 2-1, middle). The measure for this phenomenon is known as the phase locking value (PLV). 

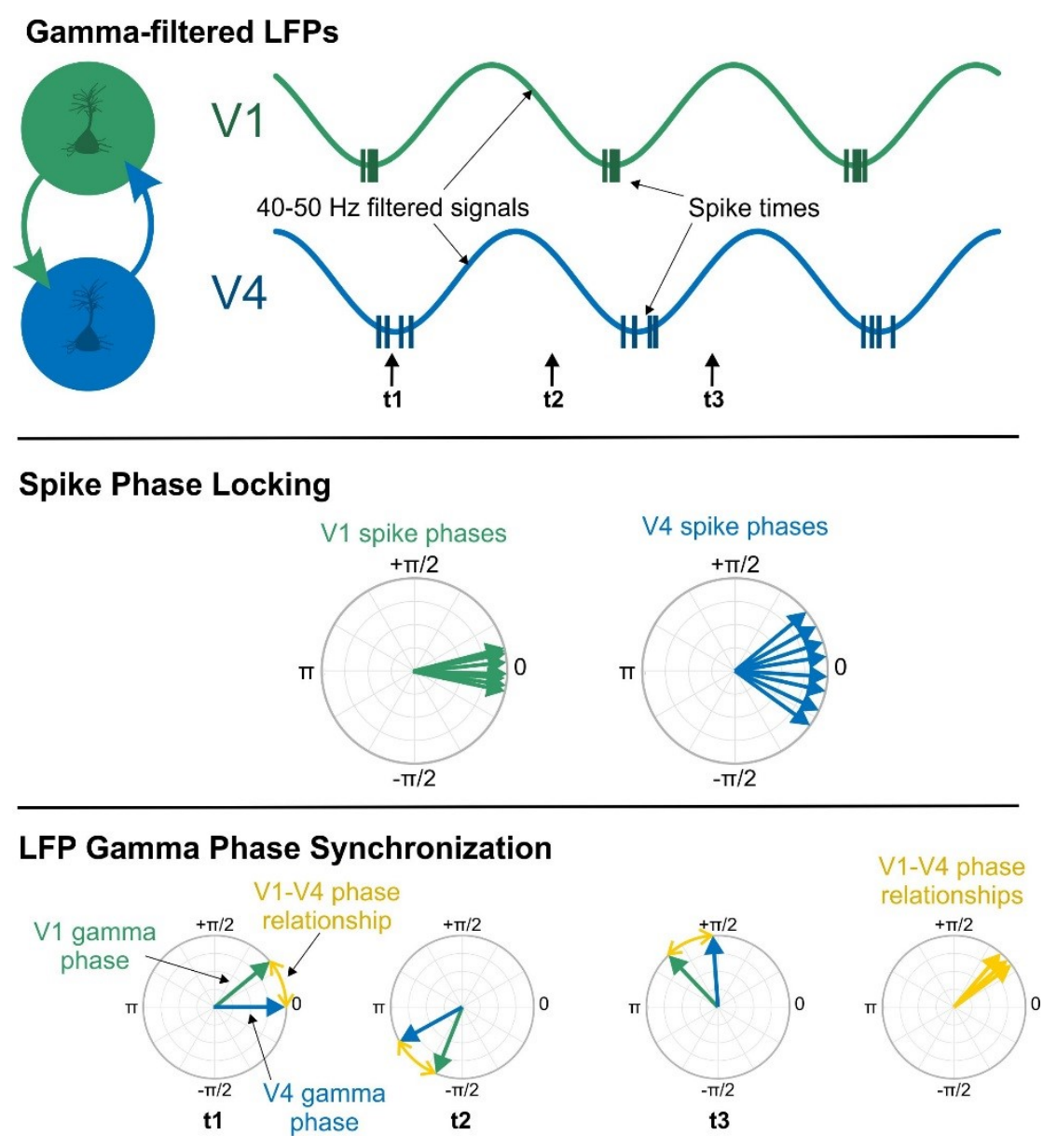

Figure 2-1. Example illustration of phase locking of spikes in the V1 and V4 LFP during gamma oscillation, and phase synchronization of gamma oscillations in V1 and V4.

Top panel shows example of filtered gamma oscillations in the 40-50 Hz frequency range occurring in V1 and V4. Spike times are superimposed on the oscillation as darkened lines, and time samples for phase synchronization analysis are indicated below the oscillations. Middle panel shows qualitative occurrence of phase locking of spikes to local gamma oscillations in V1 and V4. Arrows represent the gamma oscillation phases at the times a spike occurred. Phase locking to gamma is occurring in both regions, although a higher degree of phase locking is occurring in V1, as illustrated by the tighter clustering of spikes to the trough phase. In this example, the spike phases in V1 would yield a higher phase locking value (PLV). Bottom panel shows example illustration of gamma phase synchronization between V1 and V4. Arrows represent the gamma LFP phases at time points $\mathrm{t} 1, \mathrm{t} 2$, and $\mathrm{t} 3$, with progression in time corresponding to a counterclockwise rotation in phase. Although the gamma phases in these regions evolves rapidly over time, the difference between the two is quite stable, allowing their excitatory phases to remain synchronized. The sum of phase relationship samples can be used to create a PLV, although the statistical properties of this PLV are different from the spike PLV. In this example, the phase relationship arrows consistently point slightly positive, indicating that CTC is occurring, and directing communication from V1 to V4. 
Lastly, the differences between the gamma oscillation phases in the two regions can be calculated to create a measure known as the phase relationship (Figure 2-1, bottom). The phase relationship parameter is also angular, and can be evaluated in several ways. Foremost, since it represents the relationship between the two local network states, the phase relationship can indicate whether time windows of maximal excitability are synchronized with one another in a way that promotes effective neuronal communication between them. Typically this is analyzed over a period of time relative to an event such as the visual stimulus onset. The phase relationship angle exhibits random-looking spin while the networks are functioning independently, but stably points to a specific direction when oscillations are synchronized (Figure 2-1, bottom). This phenomenon is known as phase synchronization, and its quantification and unique statistical properties underlie many of the hypothesis tests used in the following studies. Furthermore, the direction of phase stability can be used to infer the direction of transmission, or the degree of input gain, depending on the relevant anatomy and experimental context. For example, if the V1 input to V4 is selectively ignored via a gamma mechanism, the experimenter may observe an improbably consistent phase relationship, but one that suppresses the input rather than amplifies it.

In summary, in such an example where neuronal communication between V1 and V4 is strongly supported by gamma CTC during stimulus processing, one would very likely observe all of the following: an amplitude increase in the analytic signal, a high degree of phase locking of V1 spikes toward an excitatory phase of the local V1 gamma oscillation, and a high degree of phase synchronization between V1 and V4, with V1 phase leading V4 phase by a lag that roughly corresponds to the conduction delay. The next challenge lies in quantifying synchronization appropriately and determining what a "high degree" entails for these metrics.

\section{The Synchronization Metric $r$ and Its Statistical Evaluation}

The essence of synchronization in the study of coupled systems is the alignment of phase in time, thus the instantaneous phase is the most essential parameter in the evaluation of network synchronization in the brain. The strength of synchronization is denoted by the metric $r$, and this metric can be used in both the evaluation of phase locking of spikes as well as phase synchronization of LFPs. The metric $r$ is defined as the mean resultant vector length of a set $(\alpha)$ of size $n$ angular values, each given unit length. The individual unit vectors $(v)$ and the metric $r$ are given by

$$
\begin{gathered}
v_{i}=\left[\begin{array}{l}
\cos \left(a_{i}\right) \\
\sin \left(a_{i}\right)
\end{array}\right] \\
r=\frac{1}{n} \sum_{i=1}^{n} v_{i}
\end{gathered}
$$


This equation can perhaps be demonstrated more intuitively as a process of turning the angular samples into vectors of unit length, and placing them end to end to determine if there is a directional trend (Figure 2-2). The value of $r$ can range from 0 when angles are uniformly distributed around the circle, to 1 for angles that are perfectly aligned.

The $r$ value is similar to the coherence metric in this regard, yet there are key differences. Coherence, formally magnitude squared coherence $(C x y)$, is defined as

$$
C_{x y}(f)=\frac{\left|G_{x y}(f)\right|^{2}}{G_{x x}(f) G_{y y}(f)}
$$

where Gxy is the cross-spectral density and Gxx and Gyy are the auto-spectral densities. In other words, $C x y$ detects similarities in the signals by performing a series of frequency-resolved cross correlations, both between the two signals and each signal by itself. If the cross-correlation is near as high as the signal autocorrelations, then $C x y$ will be near 1 . If the signals are dissimilar, it will be near 0 . By its formulation, the magnitude squared coherence takes into account both correlated amplitude changes and correlated phase progressions within specified frequency bands. In many cases, it is advantageous to analyze amplitude changes separately, as they may add unwanted noise to the calculation ${ }^{105}$. It is also important to note that coherence cannot be calculated across trials to determine the effect of a stimulus on oscillatory phase relationships. The $r$ metric for phase synchronization can be calculated both across a window of time to identify stable synchronization, or across trials to identify an effect of a stimulus. Therefore I will continue by using $r$ rather than magnitude squared coherence.

\section{Parametric Statistical Tests Using the $r$ Value}

The $r$ synchronization metric is descriptive, but lends itself well to a simple parametric test under certain conditions. This test is known as the Rayleigh test, with the null hypothesis being that the test set represents a sample randomly drawn from a distribution with a true $r$ of 0 (uniformly distributed). The probability of having a large $r$ value becomes increasingly small as more random samples are evaluated, thus the probability for an angular set of size $n$ with magnitude $r$ can be calculated as:

$$
p=\exp \left[\sqrt{1+4 n+4\left(n^{2}-(r * n)^{2}\right)}-(1+2 n)\right]
$$

This test is effective for determining whether there is a unidirectional bias away from uniformity, but it is also prone to false positives if not applied correctly. In many cases, the time series transformation creates a biased set of possible phase values that may not correspond to spikes or other events of interest (Figure 2-3). Because of this, time samples could be randomly chosen and still exhibit a directional bias. I will refer to this distribution as the "biased null set." Questionable significant results can also be caused by event intervals that are much shorter than oscillation intervals, although this 

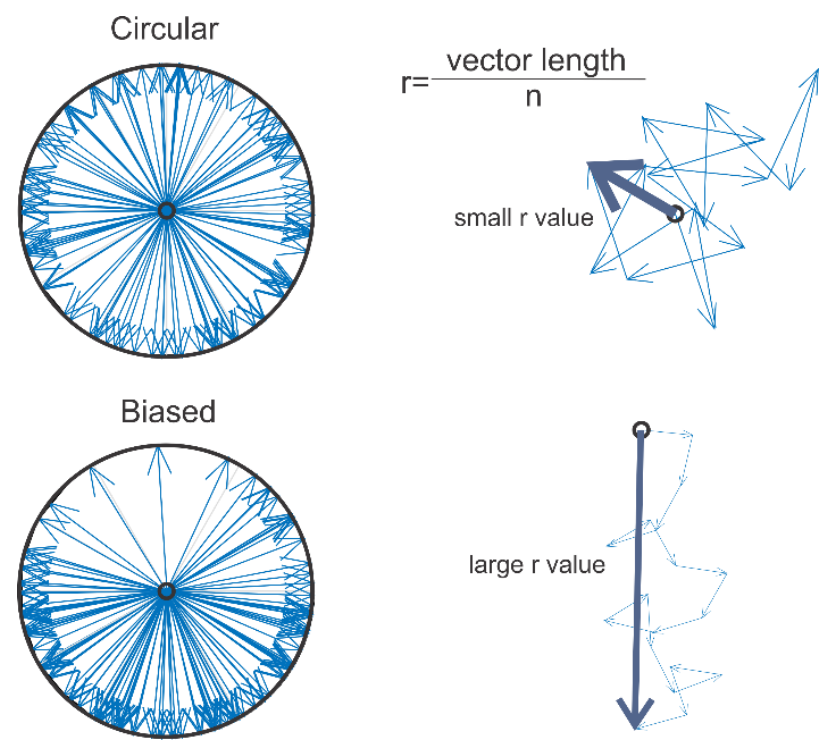

Figure 2-2. Illustration of $r$ value determination from a set of angular samples. Two example sets of angular samples are given here, one which is circular and one with a downward directional bias. In practice, these sample sets may consist of LFP oscillation phases that correspond to spike times, or LFP phase relationships following stimulus presentations. The $r$ vector represents the mean of the set of samples through vector addition. Illustration of vector addition, essentially laying the vectors end to end, is shown at right. If the set contains a directional bias, the resultant vector will be longer than if there were no bias. Thus, the length of the resultant vector and the number of samples can be used to determine the likelihood of randomly drawing a bias of a given magnitude from a non-biased data set. 


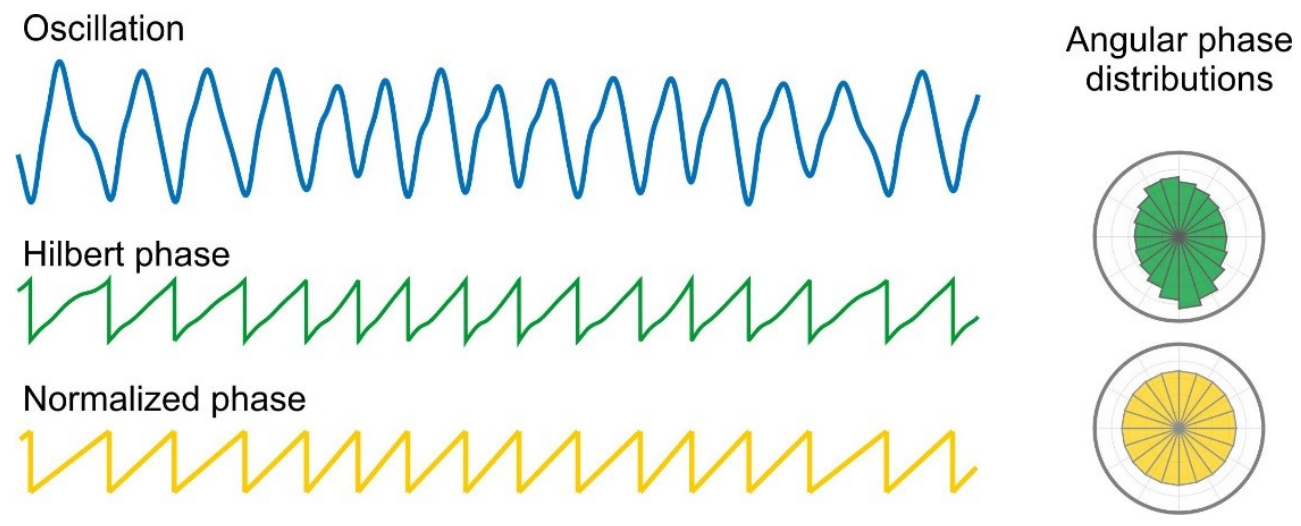

Figure 2-3. Example biased null phase distribution and phase normalization process.

It is common for oscillations to be non-sinusoidal in shape, and an example of such an oscillation is illustrated in blue. In this oscillation, more time is spent in the rising phase than near the peak or trough, creating a biased distribution of phases when estimating with the Hilbert method. This bias is shown in the green sawtooth waveform as a shallowing in the phase progression and in the phase histogram as a largely downward deviation from the center. Given this bias, random draw of time samples is likely to have a statistically significant directional bias, making it difficult to determine whether a significant spike-LFP relationship exists. To accommodate for this bias, phases can be reestimated based on peak-peak or trough-trough time, as shown in the yellow sawtooth wave. This may distort the phase estimate somewhat, but is guaranteed to generate a circular distribution from which spike phase locking can be statistically evaluated. 
may still be physiologically important, as in the case of oscillations leading to bursts of spike activity.

There are currently two ways to compensate for a biased null set in order to preserve the fast and useful parametric logic of the Rayleigh test. The first is to redefine the phase values so that they are evenly distributed across the circle. Normalizing the phase progression within each oscillation interval, or essentially defining all phases in terms of time relative to adjacent peaks can accomplish this. This can distort the phase values somewhat, but ensures that the null set of phase values is uniform so that the Rayleigh test can be correctly applied.

Unfortunately, this normalization method is not applicable to a distribution of LFP phase differences within a time segment, since its progression in time is presumably not periodic. The second method is one that addresses this problem by creating a twosample test for unidirectional deviation from a known null set. This approach has not been published in a peer-reviewed journal to my knowledge, but was proposed and formally described at least in part by Carsten Allefeld in his dissertation on phase synchronization of brain activity ${ }^{106}$. The formulation of this test was motivated by the need for a more conservative null hypothesis, since the assumption of zero synchrony in an experimental control condition is not necessarily well founded. Using the data acquired during the full recording session, one can take into account baseline levels of synchrony between the regions of interest to determine whether a stimulus or behavior drives significant changes in synchrony.

In the basic two-sample test proposed by Allefeld, two $r$ vectors are tested against one another in order to determine whether there is a statistically significant difference in the degree and direction of phase synchrony between the baseline and test conditions. This test is more conservative than the Rayleigh test, but still has notable drawbacks.

One caveat with this test is that it assumes that the null set bias is unidirectional, which may not be the case. For example, the null set may be multimodal with modal directions corresponding to different states in the recording. This may be due to different synchronization phase relationships for feed-forward and feed-back communication, or even true synchronization versus common measurement noise. Importantly, $r$ vectors and their directions are not unique to an underlying distribution, and thus calculation of the $r$ values prior to their comparison destroys potentially crucial information. As an alternative I propose a different approach: using angular binning in order to determine the unique difference in phase synchronization between conditions, and calculating an $r$ vector from that distribution (Figure 2-4). This new vector represents the degree and direction of unidirectional phase synchronization change that is uniquely associated in time with the event of interest. Furthermore, the vector's length can be statistically evaluated using Rayleigh test logic after the appropriate binning corrections have been applied $^{107}$. Implementation of this test is given in the MATLAB code in the supplemental files for this dissertation (TwoSampleBinnedR.m). 


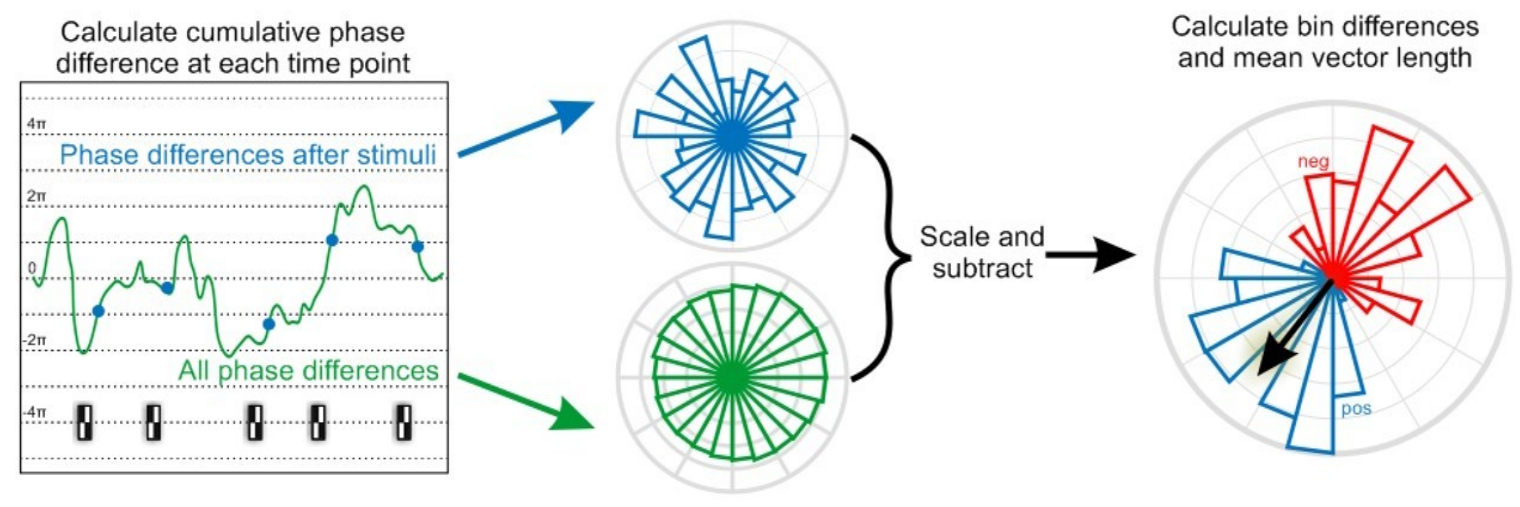

Figure 2-4. Illustration of test procedure for binned $r$ test to detect synchronization change in response to stimuli.

The phase difference time series for the entire experiment is calculated by subtracting the phase of source region from the phase of the target region. Green angular histogram represents the null distribution of phase differences, which exhibits a bias towards approximately 45 degrees. A test distribution is created by sampling phase relationships relative to the time of stimulus onset in each trial. The null set is then scaled down to have the same total number of samples as the test set, and then subtracted to create the difference distribution pictured at right. The $r$ vector calculated from the sum of the bins represents the degree of unidirectional deviation from the null distribution, and its length can be evaluated for statistical significance. 


\section{Evaluation of Binned Test Sensitivity and Accuracy}

In order to determine whether this new test is both sensitive and conservative enough in application I evaluated its performance in detecting rhythmic thalamocortical synchronization, which will be described in detail in the next chapter. Thalamocortical CTC from the lateral geniculate nucleus (LGN) to cortical layer 4 of V1 occurs in the 60$80 \mathrm{~Hz}$ range when a static high-contrast image is presented. For it to be effective, the new two-sample binned test should be sensitive to this synchronization despite possible common electrical noise at $60 \mathrm{~Hz}$. Furthermore, to remain as conservative as possible the null set should contain the test set within it so that the test statistic truly corresponds to the probability of a random draw.

To evaluate the sensitivity of the test, it will be used to detect synchronization between the two structures (Figure 2-5). To determine its statistical accuracy, one million random draws from the null distribution were performed and the correspondence of the analytic $p$ value to the actual probability of obtaining an $r$ value of a given magnitude was established (Figure 2-5a). The random draws were concurrently evaluated using the onesample Rayleigh test.

Upon evaluation, the two-sample binned test was better suited to detect changes in synchronization state following stimulus presentation (Figure 2-5b), and was also better than the 1-sample Rayleigh test at estimation of the true probability of eventrelated synchronization using the analytic $p$ approach (Figure 2-5a and c).

\section{Application to LFP Phase Synchronization Analysis}

The strong association between $r$ value and probability in the 2-sample binned $r$ test allows for accurate and relatively fast detection and statistical evaluation of eventrelated synchronization between two regions. A statistical threshold level, such as $p<0.001$, can be chosen at the test onset so that only sufficiently notable changes in synchronization state are identified in the results. Furthermore, a range of frequencies can be tested at once, and exclusively significant results can be easily mapped in a timefrequency coherogram, as shown in Figure 2-5d. To get such a result using Monte Carlo hypothesis tests would take an exorbitant amount of computation time in the case of randomization retesting for $r$ values in each frequency, and/or produce results that are difficult to interpret in the case of cluster analysis ${ }^{108}$.

While this analysis is well suited for identifying event related phase synchronization, a few caveats should be noted. When performing this analysis, it is important to keep in mind that the $r$ vector direction does not indicate the preferred phase relationship during synchronization. To determine this, one should simply use the one sample $r$ test. A sufficient number of bins are also necessary. In practice, increasing the bin number beyond 24 creates a negligible change in r value accuracy, whereas an excessively high number of bins may add considerable computation time. 
Figure 2-5. Evaluation of sensitivity and accuracy for the 2-sample binned $r$ test using randomization of real data.

Evaluation of the binned $r$ test for statistical analysis of event-related phase synchronization in the $60-80 \mathrm{~Hz}$ gamma range. Surrogate data was generated in two ways. First by randomly drawing phase values from the time series equal to the number of stimuli $(n=281)$, and secondly by randomly jittering the stimulus onset times by $+/-$ $0.5-2$ seconds to ensure samples were distributed across the time series. a) Probability distributions for $r$ values generated by randomization 1-sample and 2-sample binned tests. 1 -sample tests underestimated the $r$ value likelihood (tendency for false positives), with greater underestimation when samples are randomly drawn versus evenly distributed, likely due to changes in synchronization state over the recording period. Actual probability conformed to the Rayleigh analytic probability in the 2 -sample binned test in both surrogate conditions. b) Time-resolved evaluation of synchronization across trials using the 1-sample and 2-sample binned tests. 1-sample test is less sensitive to changes in synchrony in the 60-80 Hz range, as demonstrated by its higher pre-stimulus values and lower peak value. c) Correspondence of real and analytic $p$ values for $r$ values in panel $b$, using probability distributions for 1-sample and 2-sample binned test. Probabilities lower than 1 in one million were unable to be evaluated due to limited number of surrogate samples. 1-sample test vastly underestimates the likelihood of results, whereas binned test is largely accurate, if not slightly conservative in its estimation. d) Example application of Rayleigh analytic $p$ to create statistical threshold at $p<0.001$ in timefrequency analysis of event-related phase synchronization using the 2-sample binned test. Synchronization across the $60-80 \mathrm{~Hz}$ range is easily identified at significant at $100 \mathrm{~ms}$ from stimulus onset. Interestingly, the test also detects significant de-synchronization in the alpha range, which exhibits strong synchronization in the absence of visual contrast. 

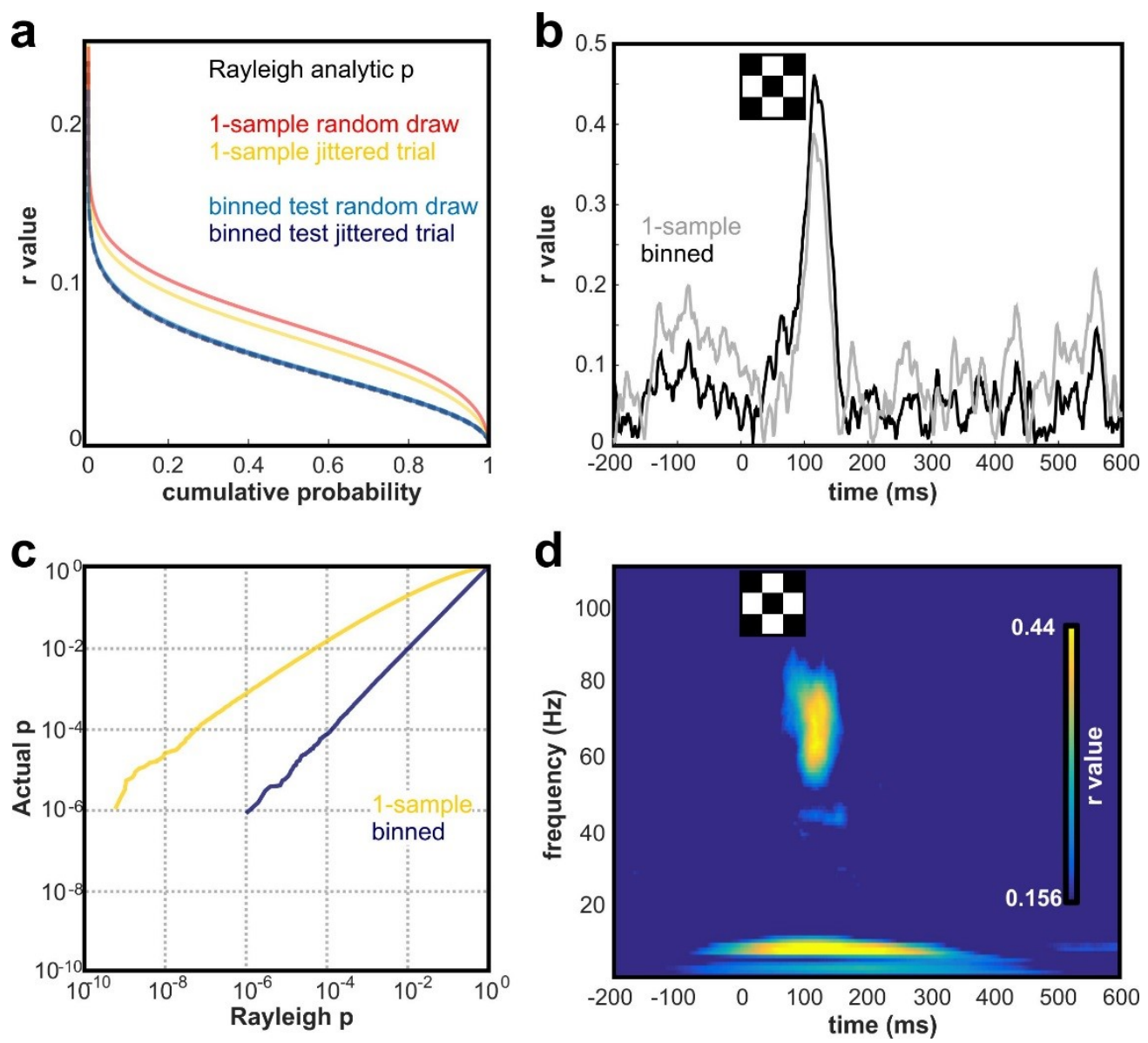
Given these characteristics, the binned 2-sample $r$ test is fast and highly effective at detecting event-related phase synchronization. For a comparison of one- and twosample statistical tests with $r$, refer to Figure 2-6.

As a final comment, while the binned test is sensitive and accurate in practice, an exact test is possible to create, if desired. In this case, one could calculate a single $r$ value from the average of the null and test vectors while giving the null vectors a magnitude of

$$
m=-\frac{n_{\text {test }}}{n_{\text {null }}}
$$

rather than unit magnitude, where $n_{\text {test }}$ is the number of events being analyzed and $n_{n u l l}$ is the number of time samples in the recording. This is somewhat impractical when determining phase synchronization in time relative to a stimulus onset, since it requires vector addition to be performed for the entire null set at each time point, which should, in good practice, consist of a large number of samples. Since the null distribution is constant, the binned approach allows for calculation that is much faster, with twice the number of calculations as the number of bins, versus the sum of $n_{\text {test }}$ and $n_{\text {null }}$ calculations at every frequency and time-step.

\section{Oscillatory Changes in Spike Synchrony}

A central assertion within the CTC hypothesis is that neuronal communication is pulsatile with respect to coherent network oscillations ${ }^{16}$. Thus, spike synchrony between sending and receiving regions should increase and decrease as a function of oscillation phase. This premise is fairly simple, yet quantifying this phenomenon with continuous LFP signals and discrete spike times can be immensely challenging, especially when the coherent oscillations occur at a high frequency.

One effective approach involves turning the two spike signals of interest into one continuous function that represents their coincidence within a physiologically meaningful time window. Once the continuous function is created, it can be used to find correlations with prominent oscillations in the LFP, or be evaluated by itself in analyses like eventtriggered averages. I used this approach to evaluate precise synchrony between LGN and V1 neurons, and relate this synchrony to LFP oscillations in V1 in Chapter 3.

\section{Creating the Spike Synchrony Function}

The spike synchrony function is essentially the difference of two simpler functions: a function that represents the spike coincidence within the time window of interest, and a subtracted function that represents the mean probability of coincidence based on spike rate. The spike coincidence function is straightforward: simply multiply the two spike counts within a sliding window, and then apply a smoothing filter of the same time width. This gives spikes that occur with more precise synchrony within the 
Figure 2-6. Illustrated example outcomes for different parametric synchronization tests in different conditions.

Left columns show binned angular distribution of phase relationship values for the null and test conditions. Arrows represent vectors to be statistically evaluated in each test. In the first two examples, when the null distribution is evenly distributed around the circle, all tests are formally equivalent. In the Rayleigh test, only the test phase difference distribution is evaluated, leading to a tendency for false positives when there is no change from a synchronized state, and false negatives when the test distribution corresponds to significant de-synchronization from baseline. The two-sample $r$ test evaluates the distance between vector endpoints in the null and test conditions, making it less prone to false positives. False negatives do occur however when the null distribution is multimodal. This can be detrimental when there is both feed-forward and feed-back synchronization, and the stimulus of interest biases the system to one state or the other, as in the fourth and fifth examples. The binned $r$ test evaluates differences in distribution shapes by subtracting null values from test values for each angular bin. This allows for sensitive and accurate evaluation of any change in synchronization state. Notably, the vector length represents the degree of synchronization change, but the direction does not always correspond to the preferred synchronization phase relationship as seen in example 5. This is because negative bin values, angles where there are fewer phase relationship samples in the test set than expected, contribute to the vector sum in the opposite direction. 


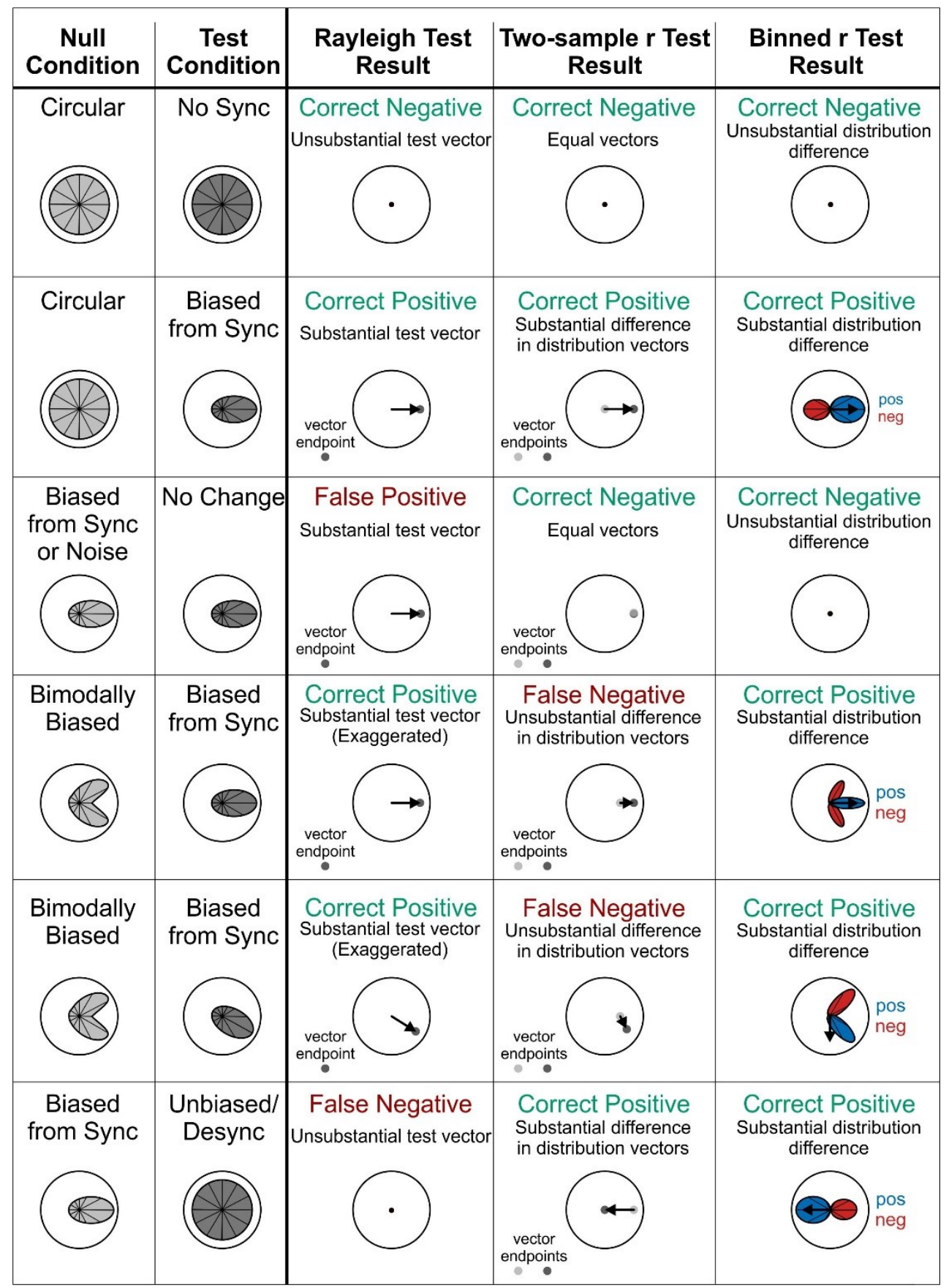


window greater weight, and also gives greater weight to multiple coincidences. Furthermore, if the conduction delay between the regions is known, then the spike time series in the sending region can be shifted by this delay to make the detection of meaningful synchrony more accurate. The correction step is essential however, as higher spike rates will inevitably lead to a higher rate of coincidence. Thus making this correction highlights coincidences that occur with high precision, above a "chance" level (Figure 2-7).

This correction method uses spike jittering as a means of estimating the likelihood of coincidence based on spike rate alone. Spike jittering for statistical estimation is very powerful in this regard, as it preserves nearly all of the temporal information in terms of spike rate and trial variability while only destroying the occurrence of precise coincidences $^{109}$. Typically, corrections are made by jittering spike times around in a window that is five times greater than the time window of interest, and subsequently performing the same analysis you would on the real data. This is then repeated many times in order to create probability distributions for coincidence at each time point, and then the median in each distribution can be used for the correction.

A much faster and mathematically equivalent approach uses what is known as "virtual jitter" to derive this median value ${ }^{110}$. In this method, the jitter time window is convolved with each spike time series, and the resulting functions are multiplied to calculate the expected rate of coincidence for each time point. When this is subtracted from the true coincidence, it reveals synchronous spikes that occur above a chance level. For implementation of this method, see the MATLAB code in the supplemental files (TimeResolvedSpikeSyncFunction.m).

\section{Statistical Evaluation of Rhythmic Spike Synchrony and CTC}

Once the spike synchrony function is created, it can be used to evaluate whether oscillations in the LFP correspond to the temporally precise co-activation of neurons in communicating regions. Since CTC is proposed to mainly affect the receptivity to input of the receiving region, the correlation of receiving region LFP with the spike synchrony function should be of primary interest. Furthermore, to determine the predominant frequency of possible CTC, a frequency-resolved cross correlation is a practical first step. An example result from this analysis is shown in Figure 2-8, left.

For statistical evaluation of a correlation, a Monte Carlo hypothesis test is necessary. Once frequencies are identified in the frequency-resolved cross correlation, the frequencies of interest should be tested individually for statistical significance. Special consideration should be taken in creating the surrogate distribution so that the results do not primarily reflect the phase locking of spikes to the LFP in the receiving region. For this reason, it is most appropriate to preserve this relationship by only jittering the spike times in the sending region when creating surrogate results. A result from this analysis is shown in Figure 2-8, right. 


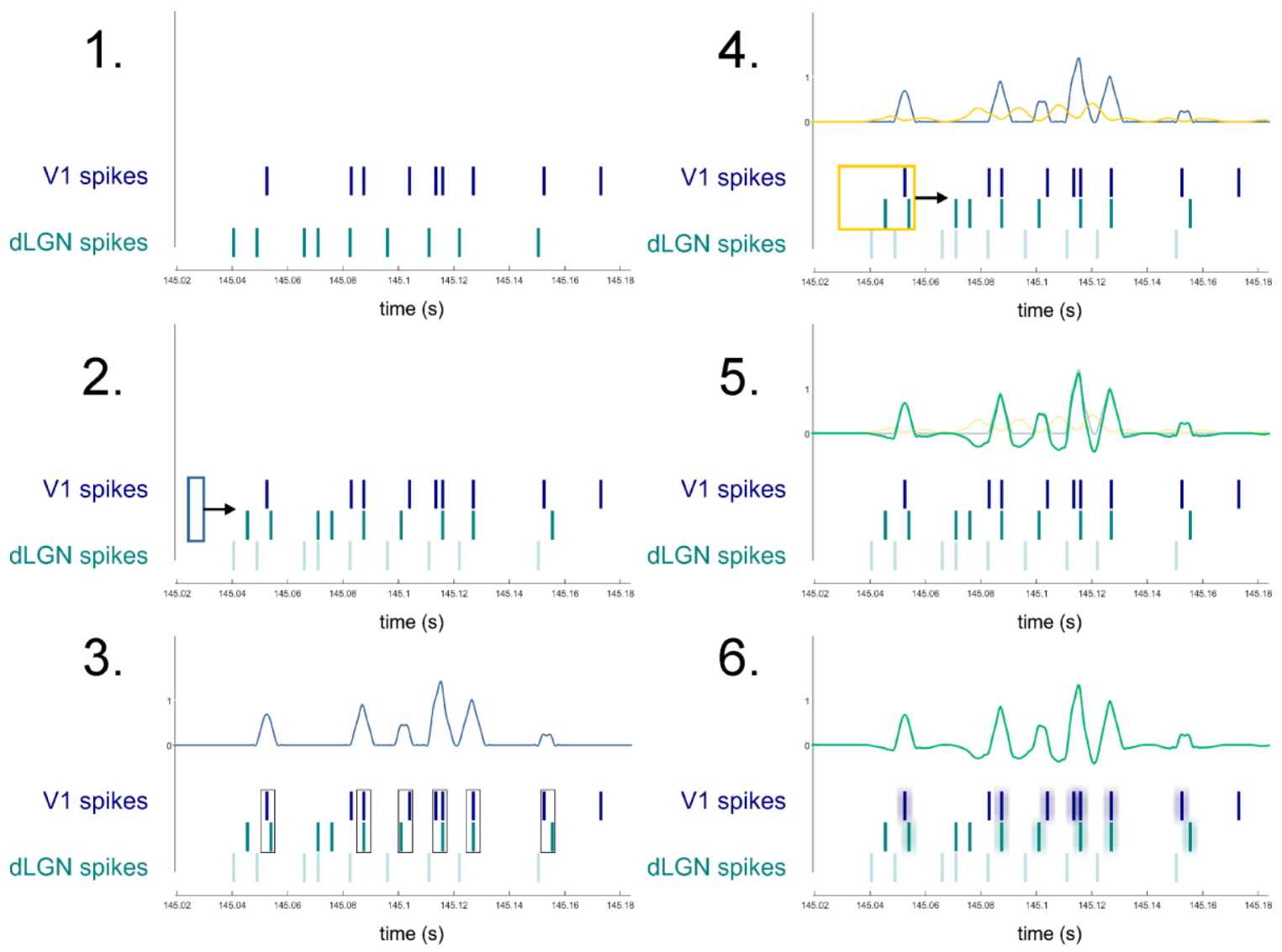

Figure 2-7. Visualization of analysis steps involved in calculating the spike synchrony function.

1. Spike times are identified within two regions of interest. V1 spikes are shown in purple, dLGN spikes are shown in teal. 2. Latency from the source region (dLGN) to the target region (V1) is known to be $5 \mathrm{~ms}$, so source region spike times are shifted forward by the latency. The timescale of synchrony that is of interest is on the order of $4 \mathrm{~ms}$, as this is the maximum interval in which synchronous output from dLGN has a cumulative excitatory effect on V1 neurons. A window of $4 \mathrm{~ms}$ width is slid across the time series, and the product of V1 and dLGN spikes within the window is calculated. 3. Smoothing of the function is performed with the same $4 \mathrm{~ms}$ time constant so that precisely synchronous spikes are given greater weight than spikes that are offset. Greater weight is also given to synchronous events with multiple spikes. Black boxes highlight six synchronous events that are reflected as positive values in the function. 4. Expected levels of spike coincidence are then calculated within a sliding window that is 5 times wider using virtual jitter. This is done in order correct for synchrony that occurs due to increases in spike rate alone. 5. The correction is then subtracted from the true coincidence to create the synchrony function. This represents a time-resolved measure of spike synchrony that is rate corrected and within a physiologically meaningful timespan. $\mathbf{6}$. The function can then be used in analysis or can be used to identify spikes that occur above chance level based on rate. 

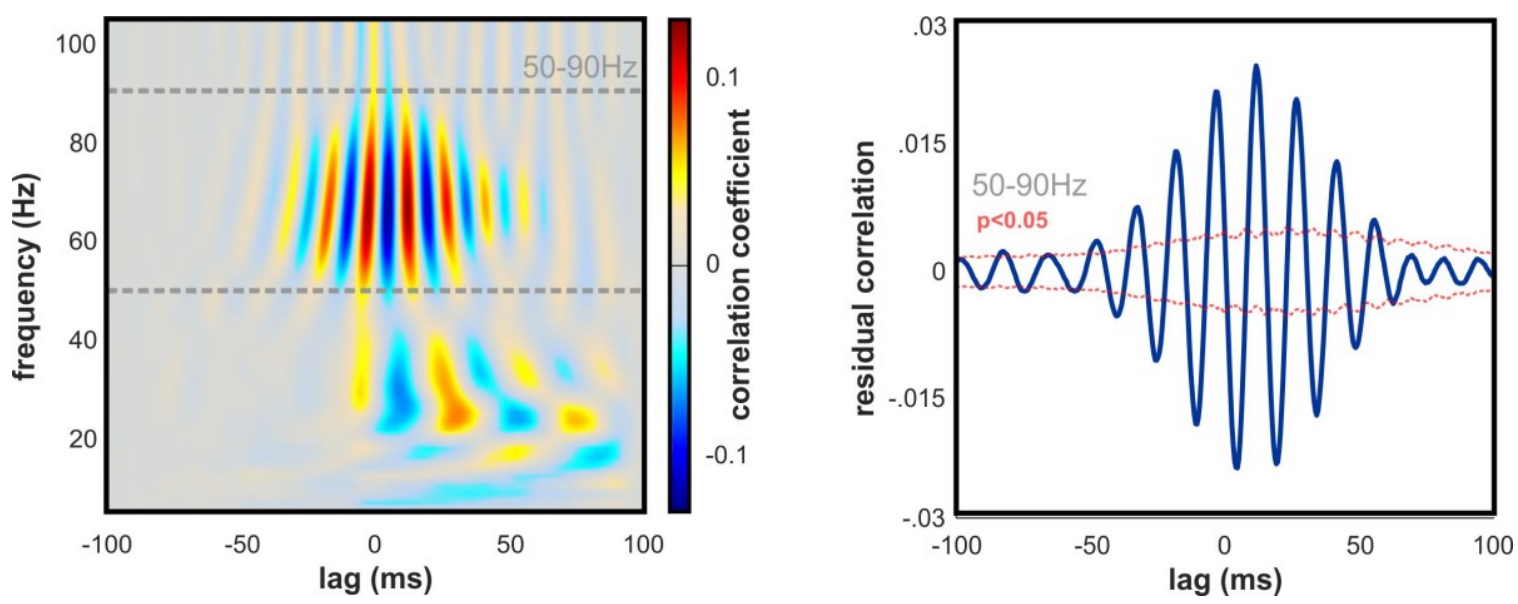

Figure 2-8. Example result of frequency-resolved cross correlation of V1 LFP oscillations with LGN-V1 spike synchrony.

At left, color plot shows correlation of the dLGN-V1 spike synchrony function with different oscillations in the V1 LFP during visual stimulation with a checkerboard image. High cross-correlation values are identified in the $50-90 \mathrm{~Hz}$ range, which are evaluated for statistical significance using a Monte Carlo hypothesis test shown at right. At right, figure shows correlation of $50-90 \mathrm{~Hz}$ component with the spike synchrony function in purple. During surrogate estimation of confidence intervals, only the dLGN spike times were jittered so that the test becomes specifically for inter-areal spike synchronization rather than phase locking of V1 spikes to the V1 LFP. Confidence bounds and cross correlation function had median surrogate values subtracted for easier visual interpretation. 


\section{Phase-Relationship Tuning of Spike Activity}

Top down influences are thought to be an important factor in establishing meaningful CTC, yet the mechanisms involved are often poorly understood. It is possible that neurons acting as high-level integrators or coincidence detectors may play a role in establishing coherence between neurons in two other regions. It is also possible that a region receiving rhythmic input from two other regions would have its activity rate modulated on the basis of coherent input. In both of these cases, an increase in spike rate in the first region should consistently correspond with specific phase relationships in the two other regions. This is the basis of the hypothesis outlined in Chapter 4, for how the cerebellum could be involved in neuronal communication between the prefrontal cortex and hippocampus.

Performing such an analysis takes computational brute force more than clever artistry. Intuitively one may think this problem lends itself to the 2-sample binned $r$ test, but variations in spike rate as well as an overlap in "null" and "test" network conditions make false positives unacceptably common. Instead, a Monte Carlo hypothesis test must be used.

As in the other cases where phase synchronization was quantified, a vector will be calculated which has a magnitude proportional to the synchronization phenomenon. In this case however, the vector corresponds to the degree of spike rate modulation in accordance with phase relationship of the two other regions in a given frequency. The vector is created by calculating the average spike rate within angular bins across the circle, and then calculating the resultant of those vector-bins (Figure 2-9). Thus, the vector length is not restricted to values between 0 and 1, and a vector with a large magnitude does not always predict a statistically significant result.

Statistically significant tuning is determined by comparing the real vector length to a distribution of surrogate vectors created by rotating the spike train in time by random amounts greater than a set minimum, such as 5 seconds. If a vector length is greater than the statistical cutoff in the surrogate distribution, typically $99^{\text {th }}$ percentile, than the spike rate tuning can be considered statistically significant. For implementation of this method, refer to the MATLAB code in the supplemental files for this dissertation

\section{(PhaseRelationshipTuning_withstats.m).}

Interpretation of a significant result is not necessarily straightforward; as mentioned earlier, spike rate modulation can represent either the cause or an effect of the phase relationship. In some examples a single neuron can exhibit phase relationship tuning to multiple frequencies of oscillation phase relationships, suggesting the possibility of a more complex arrangement of detectors and effectors. For example, it is possible that a neuron is involved in detecting phase relationships in a low frequency range and, when activated by the appropriate relationship, drives coherent oscillations in a higher frequency range. In the future, further investigation into these relationships should be conducted using optogenetic manipulations to determine a causal roles as well as directionality. 

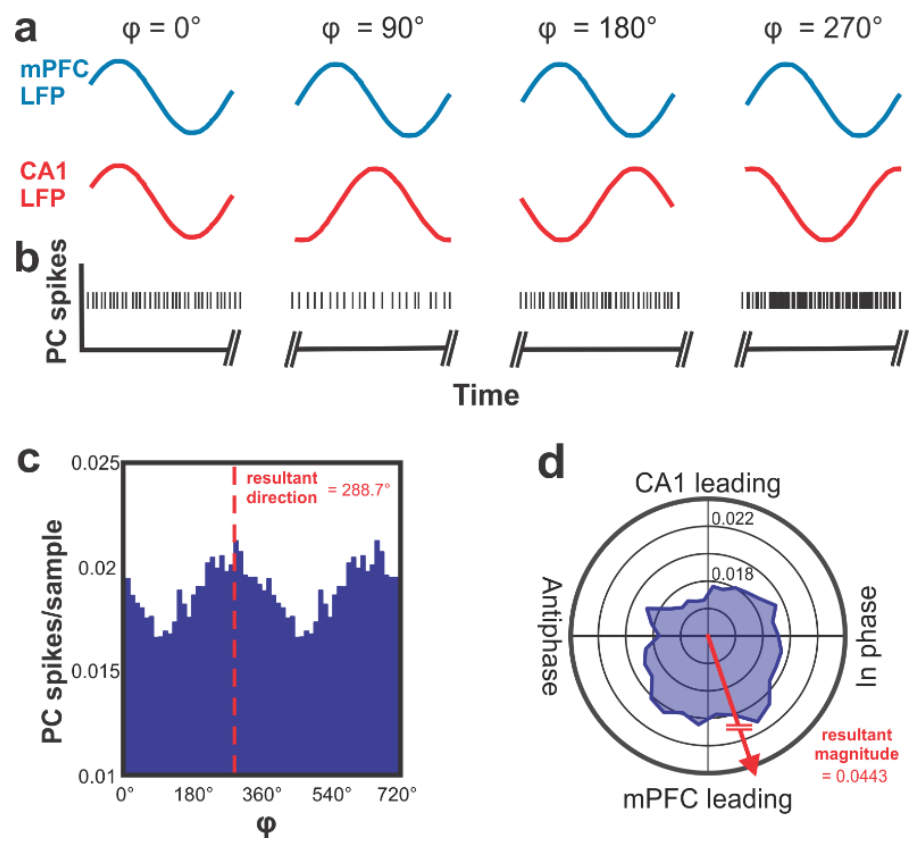

Figure 2-9. Conceptual illustration of the data analysis applied to determine the correlation between phase differences between mPFC-CA1 oscillatory LFP activity and PC simple spike activity in LS.

A: Illustration of hypothetical oscillations at a specific frequency occurring simultaneously in the mPFC (blue traces) and CA1 (red traces) and displaying different phase relationships $(\varphi)$ at different times. The phase relationship $\varphi$ is defined as the phase difference relative to the MPFC oscillation. B: Hypothetical PC spikes recorded simultaneously with the LFP activity in the MPFC and CA1 shown in a. The rate modulation of this hypothetical PC shows a significant increase in spike firing when the phase difference between $\mathrm{mPFC}$ and CA1 oscillations reaches values around $270^{\circ}$. C: Peri-phase time histogram of real PC simple spike activity. The histogram shows spike activity as a function of mPFC-CA1 phase differences at $11 \mathrm{~Hz}$. The simple spike activity of the PC in this example was significantly modulated as a function of mPFC-CA1 phase difference, with a preference of $288.7^{\circ}$. D: Same data as c represented in polar coordinates. Vectors composed of the angular value $\varphi$ and magnitude of spikes/sample were summated to determine the angular preference of PC activity. The resultant vector magnitude was taken to quantify the degree of modulation, and tested against surrogate results for statistical significance. 


\section{Phase-Amplitude Coupling}

Brain activity often exhibits a nested pattern of oscillation frequencies, with the phase of slow oscillations modulating the amplitude of higher-frequency oscillations. Several methods have been established to quantify and evaluate these patterns ${ }^{111}$, so far all of which seek to determine the persistent effect of slow oscillations on fast ones.

Of particular interest in this dissertation is the effect of respiration on brain activity. Respiration itself is a cycle with a relatively low frequency in comparison to many prominent neuronal oscillations, and its phase can be used as the "phase frequency" in PAC analysis to identify brain regions with strong respiratory-phase-to-LFP-amplitude coupling. Indeed, this has been demonstrated previously in the whisker barrel cortex of the mouse $\mathrm{e}^{90}$, and will be demonstrated in other regions in Chapter 5. This section will briefly cover the most common method of PAC quantification, and outline a new statistical method that approaches the phenomenon from a different direction.

Thus far, all methods of evaluating PAC operate under the assumption that low frequency phase is necessary and sufficient to drive the amplitude changes in higher frequency oscillations. While this has proven to be a harmless or correct assumption in many cases, it may preclude the investigator from identifying instances where the low frequency phase is one contributor amongst many in the determination of high-frequency oscillation amplitude. In other words, this means that conventional PAC analyses will reliably identify phase-amplitude relationships where low-frequency network oscillation mechanisms augment high-frequency oscillation amplitudes, but may miss cases where low-frequency phase serves to merely allow high-frequency oscillatory activity. This distinction is essential in the case of slow oscillations that bring the network closer to a critical phase transition without reliably inducing them, as is discussed in Chapter 5 with regard to respiration.

The most common metric used for investigating PAC phenomena is the Modulation Index (MI), which is similar to the resultant vector used in the analysis of phase synchrony. In this analysis, the low frequency phase and high frequency amplitude are both estimated using the Hilbert transform, and then merged to create a series of phase-amplitude vectors. The vectors are then summed to assess the degree of directional phase-amplitude bias. The resultant vector length is then statistically evaluated against a distribution of surrogates, which are generated by random time shifts of amplitude relative to phase before summation.

By comparison, the novel approach analyzes events in the inverse order by first identifying bursts of high amplitude oscillations, and then relating them to the phases of the lower frequency oscillation to discern a possible relationship (Figure 2-10). This approach uses Moore's modified version of the Rayleigh test for the analysis of vector data in order to evaluate the phase-amplitude relationship for statistical significance ${ }^{112}$. In this example, oscillation amplitudes in the gamma range are evaluated for a possible significant relationship to the respiratory phase. Only bursts of activity with 3 or more consecutive cycles above 2 standard deviations in amplitude are considered in the 

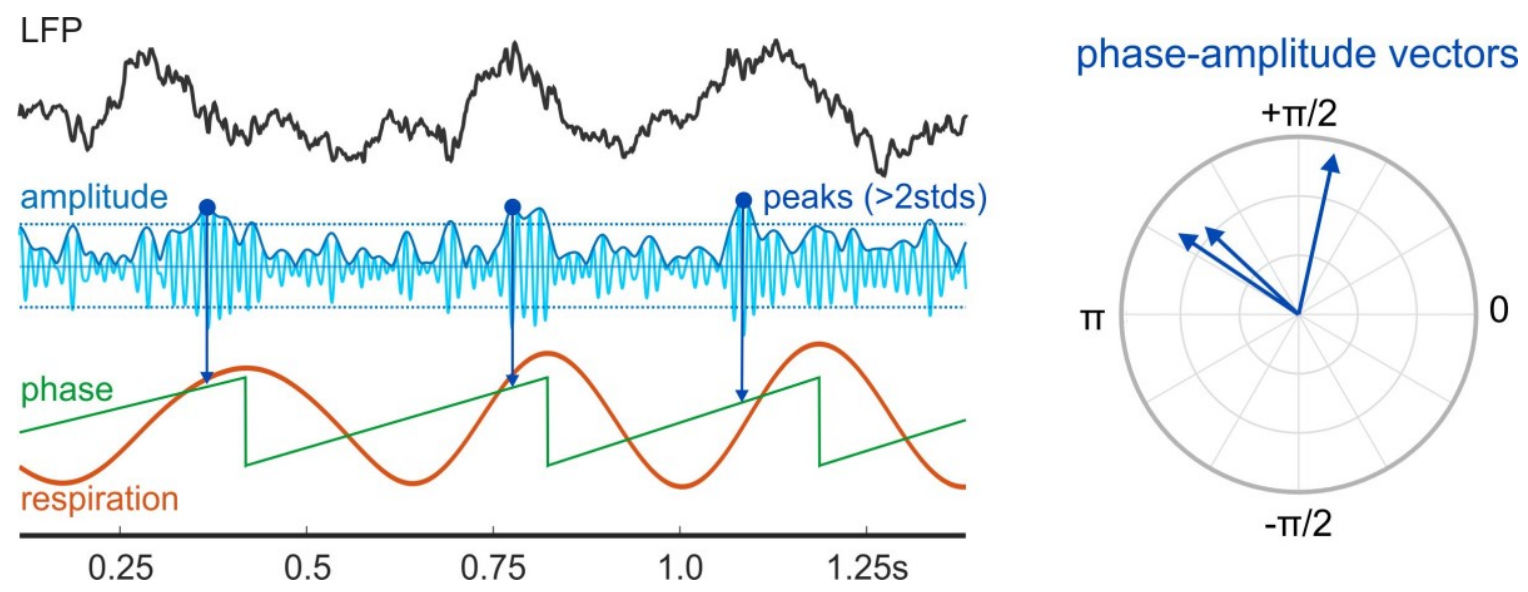

Figure 2-10. Illustration of Moore Rayleigh analysis of Respiration-LFP phaseamplitude coupling.

Respiration serves as the phase frequency in this PAC analysis. Respiration phase estimates are made using peak-peak intervals so that phase estimates are uniform across the circle. LFP gamma serves as the amplitude frequency. Amplitude envelope of gamma activity is estimated by filtering the LFP and taking the absolute value of the analytic signal returned by the Hilbert transform. Gamma bursts are identified as times when 3 or more oscillations cross the $+/-2$ standard deviation cutoff. Peak times and amplitudes of gamma bursts are then taken and referenced to the respiratory phase. At right, the vectors corresponding to the respiratory phases and gamma amplitudes are represented in a polar plot as arrows emanating from the origin. They are then statistically evaluated using Moore's modified Rayleigh test to determine whether there is a phase-amplitude relationship. 
analysis. For implementation of this method, refer to the MATLAB code in the supplemental files for this dissertation (MooreRayleighLFPAnalysis.m and accompanying files).

While this analysis constitutes a unique way to evaluate PAC, care must be taken so that it is implemented and interpreted appropriately. As with phase-locking analysis, the null distribution must be circular in order to avoid false positives. To avoid this possibility, phases in the phase frequency should be normalized within peak-peak or trough-trough times, as shown in Figure 2-3. Additionally, while the vector magnitude gives a good statistical inference, it should not be used to determine the preferred phase of amplitude potentiation. This is because the Moore-Rayleigh test changes vector values to represent magnitude rank-order, somewhat obscuring the strict phase-amplitude relationship. Instead, the simple summation of the phase-amplitude vectors will give the best representation of PAC directionality.

\section{Conclusion}

Neuronal oscillations are an essential feature of brain activity related to many important functions, and time-frequency analyses are effective at detecting these important patterns of activity. Now that the reasoning behind the CTC hypothesis has been explained, and analytic tools for assessing neuronal synchronization have been formulated, it is possible to address a range of questions related to synchronous

oscillations, behavioral events, sensory inputs, and general brain function. I will apply these tools in the following chapters to better understand neuronal synchronization and neuronal communication in different contexts. 


\section{CHAPTER 3. SYNCHRONOUS OSCILLATIONS AND THALAMOCORTICAL TRANSMISSION IN THE MOUSE VISUAL SYSTEM}

\section{Purpose}

Synchrony of rhythmic neuronal activity has been shown to be a powerful modulator of effective communication between cortical regions, yet much less is known about the role of coherent rhythms in the initial transmission from the thalamus to primary sensory cortices.

This chapter will focus on establishing the basic sets of evidence that coherent oscillations support neuronal communication between the dorsal lateral geniculate nucleus (dLGN) and the recipient layer of the primary visual cortex (V1) of the awake mouse. This includes the identification of frequency-matched oscillations, the determination that these oscillations are synchronized, and finally the determination that spike synchrony between the regions is pulsatile and aligned with these oscillations.

\section{Overview of Study}

In the neocortex, gamma frequency oscillations are involved in synchronizing spike activity within neuronal ensembles, and are believed to be crucial for the spatiotemporal organization of task-specific cortical information processing. By comparison, relatively little is known about whether gamma oscillations are involved in the transmission of sensory information from thalamus to cortex. Here, I investigated the role of gamma oscillations in promoting synchronous spike activity between the visual thalamus (dLGN) and primary visual cortex (V1) of awake mice in response to visual stimulation. Using simultaneous recordings of neuronal activity in dLGN and V1 of awake mice, I demonstrate that thalamocortical transmission of visual information involves phase-synchronized oscillations in the high gamma range $(50-90 \mathrm{~Hz})$, with highly synchronous thalamic and cortical spike activity. These results provide evidence of a role for high gamma oscillations in mediating thalamocortical communication in the visual pathway of mice, a role similar to beta oscillations in primates.

\section{Introduction}

Sensory processing requires selective and effective communication between neuronal groups within and across brain regions, and this is understood to be facilitated by the appropriate phase alignment of regional oscillations ${ }^{87}$. Connected regions can transiently increase the effectiveness of their communication (functional connectivity) by aligning their oscillations so that local neurons are driven to generate output that arrives at target regions at moments of maximal gain ${ }^{16,61,113}$. This is known as phase synchronization, and it likely underlies both the initial high-fidelity relay of information 
along a sensory pathway, as well as the recruitment of cell assemblies within a region and the selective communication among them.

In the visual cortex (V1) of a variety of species, oscillatory activity and neuronal firing synchrony are known to be stimulus-dependent, meaning there is a strong correlation of image properties such as spatial frequency and contrast with the power and frequency of cortical gamma oscillations $\mathrm{s}^{67,114,115}$, as well as long-range synchrony between neurons with similar stimulus preferences ${ }^{3,116-118}$. Inter-areal phase synchronization is most commonly studied as it applies to different cortical areas binding contiguous visual images ${ }^{11,85,119}$, but also likely plays a role in the dedicated relay of visual information from the dorsolateral geniculate nucleus of the thalamus (dLGN) to $\mathrm{V} 1^{18,120}$.

Little is known about the role of phase synchronization in thalamocortical transmission in awake animals, and the available evidence suggests there may be considerable differences in oscillation frequencies between species. In primates, experimental evidence suggests thalamocortical transmission is supported by oscillations in the beta frequency range ${ }^{18}$, with gamma oscillations first being generated in the cortex. In contrast to this, recently published studies in mice suggest that a prominent narrowband oscillation in the high gamma range may underlie visual thalamocortical transmission $^{85,121}$. This narrowband oscillation originates in the retina in response to luminance of the visual field ${ }^{122}$, and retinal activity drives coherent oscillations as far downstream as layer $2 / 3$ of $V 1^{85,121}$. Somewhat problematically for this role however, the introduction of visual contrast dramatically changes the activity in visual cortex, leading to the suppression of gamma amplitude in the narrow band and the emergence of distinct broadband low gamma and high gamma oscillations. Here it was asked whether one of these contrast-evoked gamma frequency oscillations is related to thalamocortical input. It was hypothesized that either contrast induces emergent cortical gamma oscillations, which lose alignment with narrowband thalamic input, or contrast drives similar broadband thalamic oscillations, which maintain a consistent alignment with those in cortex.

These possibilities were addressed by performing dual dLGN-V1 recordings in awake, head-fixed mice presented with zero-contrast and high contrast visual stimuli. It was asked whether high-contrast images induce synchronized gamma oscillations in mouse dLGN and V1, and investigated whether V1 oscillations were linked to the occurrence of synchronous spikes thought to reflect effective communication between the two structures. We found that the transition from the full-field gray image to a highcontrast checkerboard image lead to stimulus-locked phase synchronization of high gamma oscillations in V1 and dLGN within the range of $50-90 \mathrm{~Hz}$, immediately following the rhythmic occurrence of synchronous spike activity in both regions. These findings suggest that thalamocortical transmission involves a range of coherent high gamma oscillations, and that synchronization of high gamma is likely to constitute a dedicated frequency channel for the thalamocortical relay of visual information in mice. 


\section{Methods}

\section{Animals}

Experiments were performed on both Male and Female C57BL/6J mice $(>8$ weeks old, 18-28 g body weight). Mice were housed in a breeding colony with 12-hour light/dark cycles with ad libitum access to food and water. All mice were housed in the dark for 12 hours prior to recording. All experimental procedures adhered to guidelines approved by the University of Tennessee Health Science Center Animal Care and Use Committee. Principles of laboratory animal care (NIH publication No. 86-23, rev. 1996) were followed.

\section{Preparation for Awake, Multisite Recordings}

Mice were induced for surgery with 3\% Isoflurane (Baxter Pharmaceutical Products, Deerfield IL) in oxygen in an incubation chamber, and transferred to a stereotaxic head mount. Anesthesia was maintained with 1-2.5\% Isoflurane in oxygen through a nose cone. Isoflurane concentration was controlled with a vaporizer (Highland Medical Equipment, CA). The depth of anesthesia was adjusted until the mice failed to show a reflex withdrawal of the hind paw to a strong pinch. Blunt ear bars were used to prevent damaging the eardrums. Core body temperature, measured with a rectal thermometer, was maintained between 36.5 and $38.0^{\circ} \mathrm{C}$ with a feedback-controlled heating pad (FHC Inc., Bowdoinham, ME). Surgical techniques were described in detail elsewhere. In brief, a small craniotomy ( $2 \mathrm{~mm} \times 1 \mathrm{~mm}$ ) was made above the primary visual cortex (Bregma $-3.38 \mathrm{~mm}$, lateral $2.25 \mathrm{~mm}$ ) and dorsal lateral geniculate nucleus (Bregma $-2.18 \mathrm{~mm}$, lateral $2.0 \mathrm{~mm}$ ). The exposed but intact dura was covered with Triple Antibiotic (Walgreens, US) to maintain moisture and reduce the risk of infection. A cylindrical plastic chamber $(0.45 \mathrm{~cm}$ diameter and $4 \mathrm{~mm}$ height $)$ was placed over the skull opening and filled with Triple Antibiotic and Kwik-sil epoxy (World Precision Instruments, Sarasota, FL). Three small machine screws (1/8' dome head, $0.8 \mathrm{~mm}$ diameter, $2 \mathrm{~mm}$ long, Small Parts, Inc., Miami Lakes, FL) were secured in the skull bone, and a metal head post was mounted anterior to Bregma. The chamber, head post and skull screws were secured in place with super glue and dental acrylic. Mice were injected subcutaneously with $5 \mathrm{mg} \mathrm{kg}-1$ analgesic Carprofen (Zoetis Inc.; Kalamazoo, MI) to alleviate pain and $0.25 \mathrm{ml}$ of lactated ringer solution as a fluid supplement twice within the first $24 \mathrm{~h}$ of the surgery. Mice were allowed a 3-4 day recovery from surgical preparation before subsequent procedures.

\section{Electrophysiological Recordings}

Mice were habituated to head fixation above a freely rotating treadmill for one 30-minute session before recording sessions started. The experimental setup, headholding device and recording procedures have been described in detail previously ${ }^{123}$. Head fixation was accomplished by tightening a mechanical screw attached to a fixed bar 
through a threaded hole in the metal head post. Mice were allowed to move and walk freely on the treadmill at all times. The plastic chamber was then cleaned and filled with sterile saline solution.

LFP recordings were obtained using five hand-made glass insulated tungsten/platinum electrodes (impedance 1.0-5.0 M $\Omega$ ) guided in the ventral-dorsal direction by a computer-controlled microdrive (MiniMatrix, Thomas Recording, Germany). Stainless steel microdrive guiding tubes served as reference electrodes, and were electrically connected to the brain tissue via the electrolyte solution in the recording chamber. Guiding tubes were positioned at the surface of the brain above the targeted structures, and the electrodes were slowly advanced through the dura to their approximate target depths. Electrode positions were then finely adjusted during the presentation of visual stimuli in order to preliminarily identify the locations of electrode tips based on established patterns of evoked neuronal activity ${ }^{124}$. Electrode movements were controlled with micrometer resolution and digitally monitored (TRec EMM Client, Thomas Recording, Germany). Local field potentials and spike signals were separated by band pass filtering at 0.1 to $200 \mathrm{~Hz}$ and at $200 \mathrm{~Hz}$ to $8 \mathrm{kHz}$, respectively, using a hardware filter amplifier (FA32; Multi Channel Systems). Filtered and amplified voltage signals were digitized and stored on a computer hard disk (16 bit A/D converter; sampling rate, $>20 \mathrm{kHz}$ for action potentials, $>2 \mathrm{kHz}$ for LFPs) using a CED power1401 and Spike2 software (both Cambridge Electronic Design).

Upon completion of each recording session, electrolytic lesions were created in the tissue at the electrode tips in order to confirm recording sites. The electrodes were then retracted, saline was removed from the chamber, triple antibiotic was reapplied, and the mice were temporarily placed back into their home chambers. All mice were euthanized within 8 hours of recording and transcardially perfused with phosphatebuffered saline and $4 \%$ paraformaldehyde prior to brain tissue collection.

\section{Presentation of Visual Stimuli}

Visual stimuli were designed and presented using customized psychtoolbox ${ }^{125,126}$ and Matlab scripts. Checkerboard images (100\% contrast, $0.05 \mathrm{cpd}$, and $100 \mathrm{~ms}$ duration) were presented on a $22^{\prime \prime}$ computer monitor centered $30^{\circ}$ lateral to the animal's midline and $25 \mathrm{~cm}$ away. Stimuli were presented at randomly generated intervals ranging from 2.5 to 4 seconds. The monitor remained gray, with the same luminance as the checkerboard during the inter-stimulus intervals. Precise timing of visual stimuli was coregistered to the LFP by simultaneously recording the voltage from a photodiode placed on the corner of the screen. LFP recordings were taken on the contralateral side to the visual field in which stimuli were presented. 


\section{Analysis of Local Field Potential Amplitude and Phase}

Recorded data were visually inspected and periods that were free of movement artifact or treadmill rotation from locomotion were selected for further analysis. $60 \mathrm{~Hz}$ contamination of the signal by ambient electrical noise was removed using a humremoval algorithm in the Spike2 software (HumRemoveExpress.s2s). LFP data and stimulus times were then exported to Matlab for further analysis.

Frequency components of each LFP signal were extracted using acausal FIR band pass filters. Filters of bandwidth $0.5-5 \mathrm{~Hz}$ were used in $0.5 \mathrm{~Hz}$ steps, with filter order equal to the number of samples in 5 cycles for each center frequency. An amplitude time series for each frequency was estimated by applying the Hilbert transform to each band pass filtered signal and taking the absolute value of the analytic signal. Changes in amplitude were calculated as a percent difference from the mean values occurring within 1s prior to the stimuli. Instantaneous phase values were determined at each time point as the angular value of the analytic signal. Pairwise-phase consistency (PPC) ${ }^{127}$ values were calculated on phase relationships across trials for each frequency and latency from the stimulus in $1 \mathrm{~ms}$ time steps.

\section{Time Resolved LGN-V1 Spike Synchrony}

In order to assess the connection between oscillatory synchrony and neuronal communication between V1 and dLGN neurons, I focused on the occurrence of synchronous firing of single units between the regions. Single units were detected using the offline spike sorting algorithm in Spike2 (CED details), and spike times were exported to Matlab for further analysis.

A major aim in this study was to determine whether rhythmic occurrences of synchronous spikes could be detected in the recording in a manner that allowed for later decomposition into frequency components. This type of approach would require a conversion of discrete spike events into a continuous function, which allows for interpretation of individual stimulus presentations as well as averaging across them. To accomplish this, a continuous time series was created to reflect spike synchrony by sliding a window across the recording period and measuring the coincidence of $\mathrm{V} 1$ and dLGN spikes (e.g. the product of their counts) within the window. Times of dLGN spikes were shifted by $5 \mathrm{~ms}$ to account for the known conduction delay, and measured coincidences within a conservative $4.5 \mathrm{~ms}$ time window roughly corresponding to the interval within which synchronous output from dLGN evokes maximal V1 responses. The mean result derived from 200 surrogate data sets with random spike jittering was then subtracted in order to correct for synchrony occurring due to increases in spike rate alone ( $\pm 2-10 \mathrm{~ms}$ jitter range, $0.5 \mathrm{~ms}$ resolution, uniform probability). The jittering method was found to be preferable to using a trial shift or shuffle predictor for this correction, as it disrupted only the precise timing of spikes while preserving the approximate latency and specific spike count in each response. The function was then smoothed with a $4.5 \mathrm{~ms}$ window. This process revealed rhythmicity in the synchronous occurrence of spikes, 
which could then be the subject of individual trial investigations as well as timefrequency analysis in the same manner as the LFP.

\section{Correlation of Spike Synchrony and Gamma Activity}

Lastly, I investigated whether synchronously occurring spikes in dLGN and V1 were consistently linked to the occurrence of V1 oscillations at different frequencies. To do this, the synchrony was used to perform a cross correlation analysis with different band pass filtered components of the V1 LFP. Sections corresponding to gray screen and checkerboard viewing were separated before further analysis in order to find differences between the two states.

\section{Experimental Design and Statistical Analysis}

Six mice ( 2 female, 4 male) underwent successful electrophysiological recording in the current study. Successful recording was defined as having one or more units in each location that responded positively to contrast increase, having at least 250 artifactfree stimulus cycles executed over a 15 minute recording session, and having histological confirmation of recording in dLGN and layer 4 of V1. Twelve mice underwent at least some portion of the aforementioned surgical and experimental procedures but failed to meet these criteria.

All statistical analyses were performed in Matlab. Statistical assessment of correlations between LFP oscillations and spikes was performed using non-parametric Monte Carlo approaches with surrogate data. For assessment of the correlation between PPC and V1 oscillation amplitude, surrogate values were generated for each evaluated frequency by randomly shifting PPC results across time in a circular manner with respect to the mean LFP amplitude of the same frequency. 200 surrogate values were generated for each frequency for each animal. The surrogate coefficients were then ranked, and the 95th and 5th percentile coefficients for each frequency were used as a statistical threshold. Averaging of correlation values for graphical representation was performed following a Fischer transformation of the coefficients, and then the inverse on the mean. $\mathrm{R}^{2}$ values for the peak V1 high gamma frequencies were created by taking the square of the raw coefficient, and averaged across animals.

For analysis of the relationship between LGN-V1 spike synchrony and the V1 LFP, a Monte Carlo hypothesis test for the precision of spike times and their temporal relationship to V1 oscillations in the high gamma range $(50-90 \mathrm{~Hz})$ was developed and implemented. For this approach, real correlations were tested against a ranked distribution of surrogate correlation values between the V1 LFP and jittered spike synchrony waveforms. In order to conservatively test for a relationship between thalamocortical spike synchrony and V1 gamma without bias caused by the phase locking of V1 spikes to V1 gamma, only dLGN spike times were jittered in the generation of 
surrogates. Correlation values above the 95 th percentile or below the 5 th percentile were considered significant.

\section{Data Availability}

The data generated during this study are available from the corresponding author upon request.

\section{Results}

\section{Visual Stimulus-Driven Gamma Oscillations in dLGN and Layer 4 of V1}

As reported in other studies, I observed high amplitude gamma oscillations in layer 4 of V1 in response to the presentation of a high-contrast checkerboard image as well as "narrowband" gamma oscillations during periods of viewing the gray screen (Figure 3-1). By comparing contrast-evoked oscillations to the amplitudes of corresponding oscillations in the gray screen condition, it was possible to identify activity changes associated with contrast onset, and characterize the rapid transition between the two states (Figure 3-1C, D, E, F, and G). V1 showed a dramatic amplitude increase in the high beta/low gamma $(20-35 \mathrm{~Hz})$ and high gamma range $(50-90 \mathrm{~Hz})$, the latter of which was higher in frequency and had broader bandwidth than the previously reported narrowband gamma. The dLGN LFP showed no such increase in beta or low gamma but did show increased high gamma amplitude, with frequencies overlapping with gamma oscillations in V1. Time-frequency analysis revealed that high gamma oscillations arose in dLGN before V1, consistent with a causal role.

\section{Phase Synchronization and Amplitude of LFP Oscillations}

Gamma oscillations in the cortex are most commonly generated and maintained by local cycles of inhibitory activity ${ }^{54}$, however, narrowband high gamma activity in V1 was shown to be driven by excitatory input from dLGN, even when cortex was silenced $^{121}$. To explore the hypothesis that broadband high gamma oscillations occurring in V1 were driven by rhythms in dLGN, I investigated which V1 oscillations concurrently increased in amplitude while phase coupling with frequency-matched dLGN oscillations. In order to do this, a correlation analysis was performed on the average time course of phase synchronization (PPC) and the amplitude of oscillations in V1, a similar approach to what was developed for a comparable investigation in primates. This analysis revealed that V1 high gamma amplitude co-varied with the strength of phase synchronization with dLGN (significant in 6 of 6 animals), with dLGN-V1 phase synchrony accounting for $66.9 \%$ of the amplitude in the peak V1 high gamma frequency across animals. A consistent inverse pattern was found in the alpha range, such that alpha 

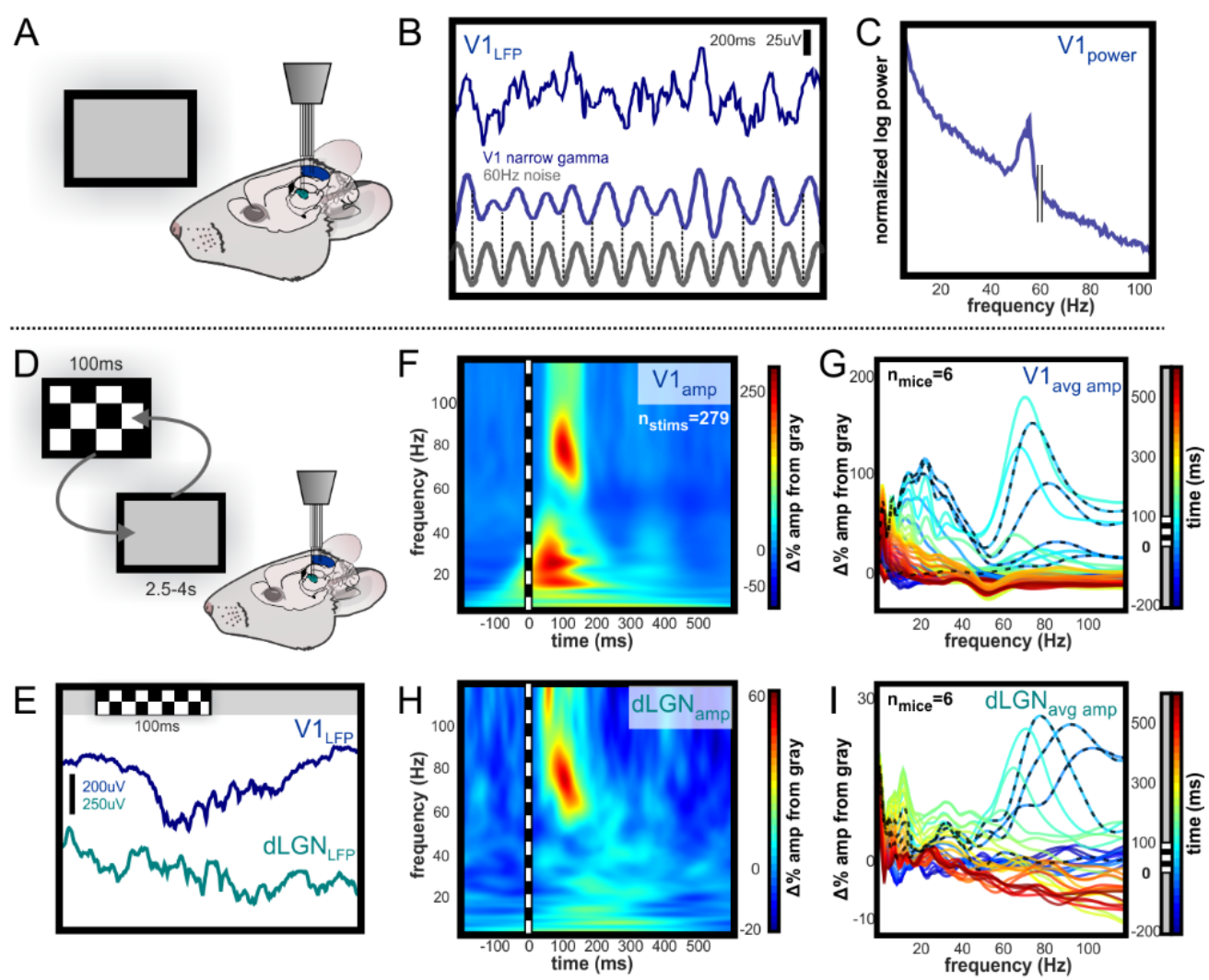

Figure 3-1. Visual-evoked narrowband and broadband high gamma oscillations in the visual system.

Independently guided electrodes were used to record LFP and spikes from L4 of V1 and dLGN during visual stimulation. A: Illustration of experimental setup during baseline recording of activity evoked by viewing of the gray computer screen. B: Example of $200 \mathrm{~ms}$ of raw narrowband gamma in L4 of V1 evoked by luminance of a gray computer screen. The frequency is between 50 and $60 \mathrm{~Hz}$, but ambient $60 \mathrm{~Hz}$ electrical noise is not linked to this oscillation, as illustrated by the phase shifts near the beginning and end of trace. C: Spectral power of narrowband gamma oscillation in V1. Peak in spectral power is apparent below $60 \mathrm{~Hz}$. D: Illustration of experimental setup during randomized presentation of high contrast checkerboard image E: Brief high-contrast checkerboard stimuli were presented and changes in oscillatory activity were observed in V1 and dLGN. Contrast-evoked oscillations are higher in amplitude and wider in bandwidth than narrowband gamma in V1, and are also visible in dLGN. F: Example time-frequency analysis of contrast-evoked LFP oscillations in V1, normalized to amplitude during gray screen viewing. 100ms checkerboard image evokes high amplitude oscillations in distinct low and high gamma frequency ranges. G: Group mean change in oscillation amplitude over time relative to LFP during gray screen viewing. Each line represents amplitude within a $25 \mathrm{~ms}$ time bin. Peak amplitude change occurs $100-125 \mathrm{~ms}$ after stimulus onset in the $50-90 \mathrm{~Hz}$ range. Narrowband gamma remains decreased immediately after checkerboard offset. H,I: Same as D,E for LFP in dLGN. Low gamma oscillation amplitude is not modulated in dLGN, and high gamma oscillations arise in LGN before V1. 
oscillations in V1 became uncoupled from those in dLGN as contrast drove alpha amplitude increases in V1 (significant in 6 of 6 animals; Figure 3-2C and D).

\section{dLGN-V1 Spike Synchrony}

Sudden changes in visual stimuli are often characterized by rapid burst-firing of neurons in the dLGN, which we observed in response to the checkerboard image. Onset of contrast-induced spiking in V1 units typically occurred at the offset of bursting in dLGN units, and these initial spikes were synchronized with those in dLGN above mean probability based on spike rate alone (Figure 3-3).

Occurrence of synchronized spikes began after initial dLGN bursts, but before the onset of high amplitude broadband gamma in V1. Time-frequency analysis of the spike synchrony revealed peak rhythmicity in the high gamma range, which overlapped with the frequency of evoked gamma oscillations in V1 (Figure 3-4). The duration of this rhythmic interaction overlapped with the emergence of high gamma in the V1 LFP. Broad stimulus-evoked spike synchrony between visually-responsive neurons has been reported in mouse cortex at recording sites separated by a distance of $350 \mathrm{um}^{116}$ to $500 \mathrm{um}^{85}$. Based on estimations from published images of intact thalamocortical projections in vitro ${ }^{128}$, the length of visual thalamocortical axons, which project to the cortex via the white matter tract along the dorsolateral edge of the hippocampal formation, is within the range of 5000-6500um. This is a far greater length than the broadest width of the V1, thus the precise synchrony observed between thalamic and cortical neurons spans a distance greater than any reported primary cortico-cortical connection.

\section{dLGN-V1 Spike Synchrony and V1 Oscillations}

In order to determine whether rhythmicity of synchronous spikes in the thalamocortical pathway were linked to LFP oscillations in V1, a correlation analysis was performed between the synchrony function and different frequency components in the LFP. This analysis was performed separately for periods where the animals were observing the gray and the checkerboard images. Although spike rates in V1 were lower during gray screen viewing, periods of spontaneously increased spike activity showed rhythmic spike synchrony which was highly correlated with narrowband gamma in V1 (Figure 3-5A). Similarly, rhythmic spike synchrony that occurred time-locked to the presentation of the checkerboard image was highly correlated with gamma oscillations across a broader frequency band, which overlapped with the evoked broadband gamma, supporting a causal relationship (Figure 3-5B). Correlation values were statistically significant in the high gamma range during visual stimulation with the checkerboard in 6 of 6 mice (Figure 3-5D), and during gray screen viewing in 5 of 6 mice (Figure 3-5C), based on the results of our Monte Carlo hypothesis test. 

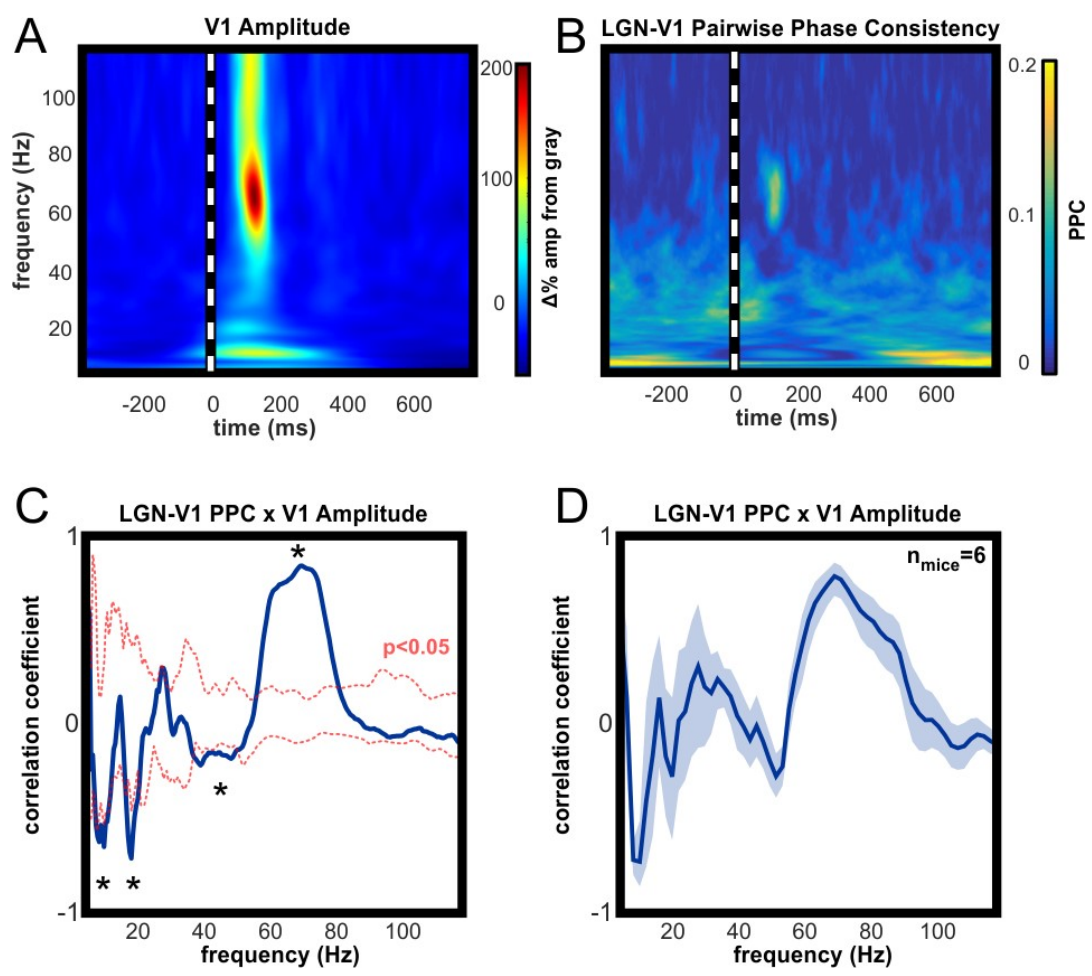

Figure 3-2. Amplitude of high gamma oscillations is strongly linked to simultaneous thalamic high gamma activity.

A: Example time-frequency analysis of V1 LFP, aligned on the presentation of $100 \mathrm{~ms}$ full-contrast checkerboard image. B: Example time-resolved pairwise phase consistency analysis of oscillation phase synchronization between dLGN and V1. PPC is increased in the high gamma frequency range following checkerboard stimulus. C: Correlation analysis of A and B to identify V1 oscillations whose amplitude is dependent on synchronous thalamic oscillations across stimulus conditions. High gamma in V1 is strongly correlated with synchronous oscillations in dLGN, and strongly anti-correlated with synchrony in the alpha, beta and low gamma ranges. D: Group mean results $( \pm \mathrm{SEM})$ for correlation analysis in C. All animals had significant correlations within the $50-90 \mathrm{~Hz}$ high gamma frequency range and significant inverse correlations in the alpha frequency range. At the peak high gamma frequency in V1, dLGN-V1 phase synchronization accounted for an average of $66.9 \%$ of the amplitude modulation, across animals. 

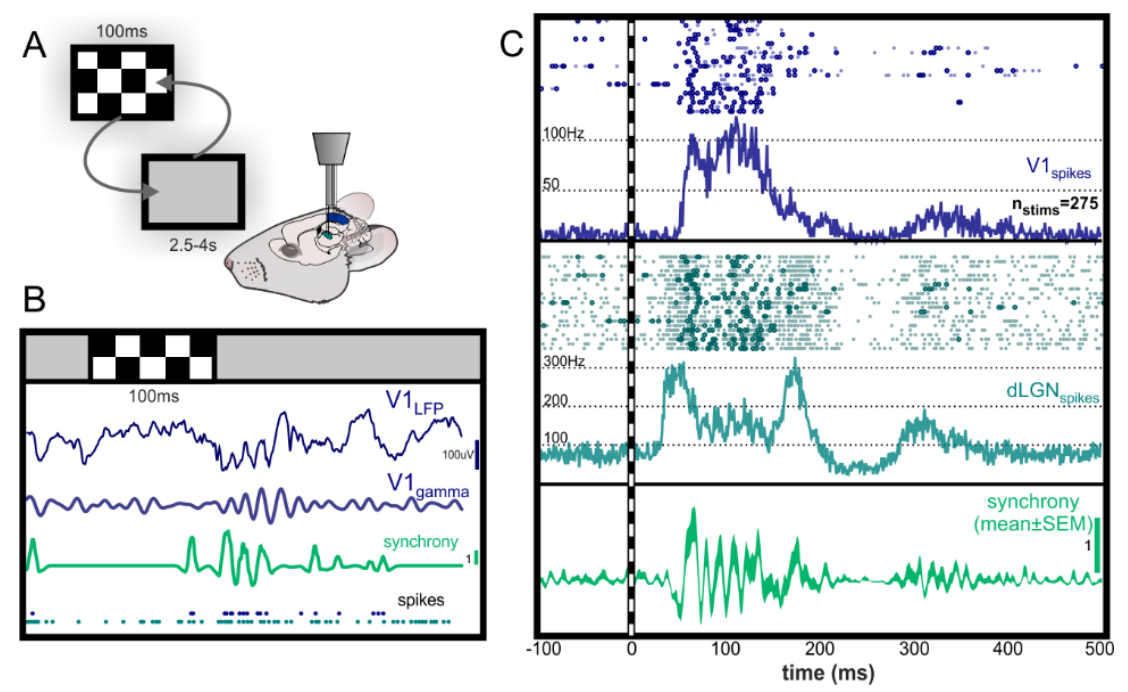

Figure 3-3. Time-resolved estimation of spike synchrony is rhythmic in the high gamma range.

A: Illustration of experimental setup during randomized presentation of high contrast checkerboard image. B: Single trial example of spike synchrony following checkerboard stimulus. Green trace represents time-resolved spike synchrony function, calculated from V1 and dLGN spike times. Synchronous bursts of spikes are observed following the checkerboard image. Scale bar shows height of 1 coincidence above mean probability. C: Example raster for V1 and dLGN spikes occurring in 20 consecutive trials, and peristimulus histogram over all trials. Circles in raster highlight spikes occurring synchronously above mean probability. After initial dLGN burst and V1 response, dLGN spikes appear to be modulated at an approximate $68 \mathrm{~Hz}$ rhythm. Bottom panel shows synchrony function averaged over trials to reveal rhythmicity of synchronous spikes locked to the stimulus onset. 

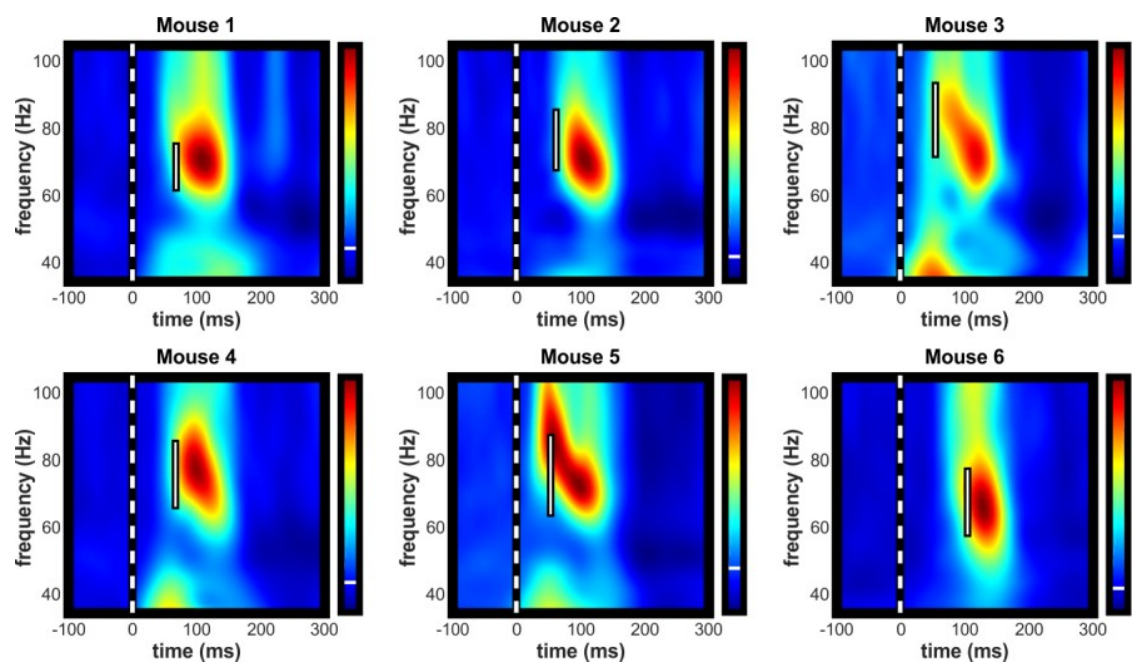

Figure 3-4. Amplitude increase of high gamma oscillations in V1 LFP occurs after synchronization of spike activity in the same frequency range.

Individual examples of contrast-evoked gamma oscillations with latency and bandwidth of peak spike synchrony superimposed. White bar spans the bandwidth of $95 \%$ maximal amplitude of rhythmic spike synchrony. All color axes are normalized to maximum amplitude increase and decrease with white line indicating zero amplitude change. 

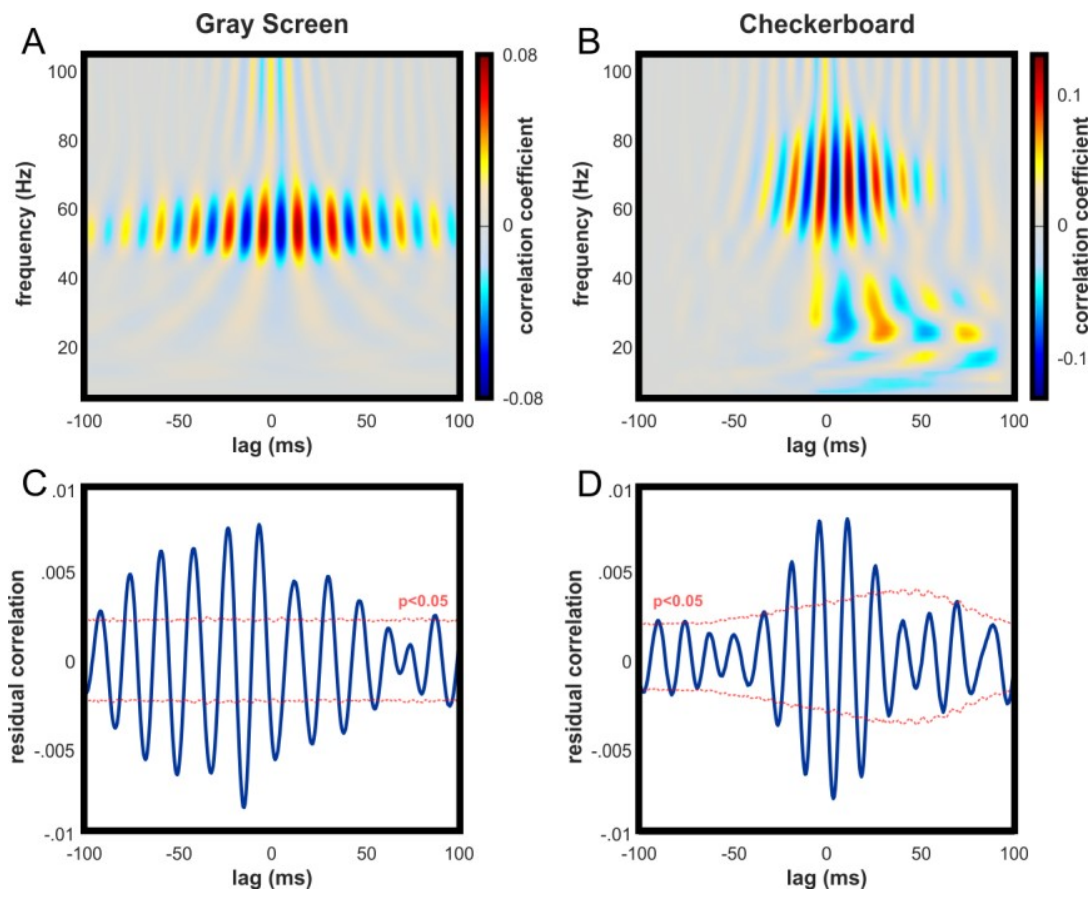

Figure 3-5. Rhythmic synchronization of spike activity in dLGN and V1 is strongly linked to both narrow and broadband high gamma in V1.

A: Example cross-correlation of dLGN-V1 spike synchrony and V1 LFP oscillations across frequency spectra during gray screen viewing. Peak correlation occurs in the narrowband gamma frequency range. B: Same as A for checkerboard viewing. Peak correlation exists across a broader frequency band, consistent with contrast-evoked broadband high gamma in V1. C: Example statistical test for correlation between spike synchrony and bandpass-filtered high gamma in the V1 LFP $(50-90 \mathrm{~Hz})$ during gray screen viewing. Tested individually, significant peaks and troughs were detected in 5 of 6 animals. 1000 surrogates were generated by jittering dLGN spikes and median surrogate values were subtracted for easier visual interpretation. D: Same as $C$ for checkerboard viewing. Tested individually, significant peaks and troughs were detected in all animals. 


\section{Discussion}

Simultaneous recordings of local field potential and spike activity in six mice revealed consistent patterns of rhythmic interactions between V1 and dLGN associated with neuronal processing of images with changing contrast. Our findings support the hypothesis that both narrowband and broadband oscillations in the high gamma range in V1 are associated with the effective thalamocortical transmission of visual information. The transition from a gray screen image to a high contrast checkerboard image was characterized by a rapid sequence of events: first, bursting of thalamic cells in response to contrast onset, followed by the rhythmic occurrence of spikes in V1 and dLGN in the high gamma range. This gamma-locked spike synchrony was followed by an increase in the amplitude of gamma oscillations in V1, which were phase-locked to gamma oscillations in dLGN. The center frequency of the high gamma response in V1 varied between mice, but had $20-30 \mathrm{~Hz}$ bandwidth within the frequency range of $50-90 \mathrm{~Hz}$. Across stimulus conditions, the amplitude of high gamma in V1 was strongly linked to the degree of phase synchronization with high gamma in dLGN, and the occurrence of synchronous spikes between the regions was significantly correlated with oscillations in the high gamma range of the V1 LFP.

Prominent contrast evoked beta/gamma oscillations in V1 below the narrowband high-gamma frequency were also observed, which were not synchronized with activity in dLGN. Recent reports show that V1 oscillations in this frequency range were evoked by high contrast bars, and that the amplitude of these oscillations corresponded to the contiguous width of the bars ${ }^{85}$. Given that LFP is an aggregate measure of neuronal currents in a recording area ${ }^{22}$, this is consistent with the hypothesis that the low gamma observed in V1 in response to a broad checkerboard image reflects cortico-cortical interactions independent of rhythmic thalamocortical influences. The evidence that these oscillations were mediated by local somatostatin-expressing interneurons further supports this as an emergent cortical phenomenon.

\section{Comparison to Awake Primates}

Previous studies in both rodents and primates have shown that LFP oscillations in separate frequency bands allow for single regions to selectively communicate with multiple others, as if by dedicated "channels" for transmission of sensory information ${ }^{17,18}$. In the context of visual processing in primates, these distinct frequency bands are thought to correspond to mechanisms of top-down versus bottom-up signaling between lower- and higher-order visual cortical regions ${ }^{11,19,20}$. Interestingly, gamma oscillations were found to first appear in V1, and are driven by the strength of synaptic

input from $\mathrm{LGN}^{67,129}$, which occurs rhythmically in the beta frequency range ${ }^{18}$.

Our results suggest that the rhythms that support thalamocortical communication are substantially different in rodents and primates, and that oscillations in mouse V1 in the high gamma range are not an emergent phenomenon, but are driven by rhythmic input from dLGN. Previous studies which explored the generation and function of gamma in 
the mouser retina, dLGN, and V1 strongly support this difference between species $^{121,122,130}$.

Local field potential components in the high gamma range are known to arise in macaque visual cortex during increased spiking activity from visual stimulation, and have been studied independently of thalamic activity ${ }^{129,131}$, but there are diverging hypotheses about their role in visual processing. One group determined through an information theoretic analysis that power in the high gamma band (peak at $\sim 74 \mathrm{~Hz}$ ) conveyed the most information about the visual stimulus being presented ${ }^{131}$. The amplitude of these oscillations clearly co-varied with the visual-evoked spike rate, suggesting a link between high gamma in V1 and excitatory input related to visual information. Such a role for high gamma in monkeys - namely that it is associated with the fidelity of visual information relay to cortex - is consistent with our findings, although the mechanisms remain obscure. In contrast to this interpretation however, another group made the observation that these visual-evoked frequency components are mostly transient (rather than oscillatory) and not band-limited, suggesting that they are most likely a reflection of currents generated by the firing of local units ${ }^{129}$. This is not consistent with our observations in mice, given that oscillations in the high gamma range are band-limited in both the amplitude change (Figure 3-1F and G) and the phase synchrony with dLGN (Figure 2C and D). Given these considerations in addition to the direct study of simultaneous LGN and V1 recordings in macaque, it seems unlikely that high gamma in V1 is associated with thalamocortical transmission of visual information in primates.

Rhythmic synchronization of neuronal activity as a mechanism to support thalamocortical transmission has been investigated previously in cats as well, although these experiments were performed under anesthesia ${ }^{120}$. To briefly summarize these results, rhythmic spike correlations within LGN exist exclusively in the high gamma range $(>70 \mathrm{~Hz})$, and thalamocortical spike correlations have been observed over broad frequency range $(30-100 \mathrm{~Hz})$ with a median frequency of approximately $50 \mathrm{~Hz}^{120}$. Rhythmic synchronization was observed between visual cortical neurons at lower frequencies $(30-50 \mathrm{~Hz})^{120}$, consistent with findings in anesthetized rodents ${ }^{116}$. This may suggest a role for high gamma synchronization in dedicated thalamocortical communication in cats, but further investigation is necessary. To our knowledge, no studies of simultaneous recordings from LGN and V1 have been conducted in awake cats, confounding a direct comparison to our findings.

\section{Broad Synchrony between Thalamic and Visual Cortical Units}

The reliability with which significant spike correlations were captured seems worthy of consideration. The fact that all mice exhibited prominent broadband gamma in $\mathrm{V} 1$, as well as precise spike correlations between neurons separated by a projection of several millimeters, suggests that broadband gamma driven by stimulus contrast likely plays a role in the synchronization of extensive neuronal populations across these regions. This is consistent with the hypothesized function of thalamocortical circuit architecture, which includes several features that promote synchrony on a broad scale ${ }^{132}$ 
and maximize the effectiveness of information relay ${ }^{133}$. These features include stimulusdriven inhibition from the thalamic reticular nucleus, which is activated by both cortical and thalamic neurons and synchronizes spike output from the thalamus ${ }^{134}$. Furthermore, in mammals such as cats and monkeys, a subpopulation of thalamocortical neurons are broadly-projecting and nonspecific, with the hypothesized role of creating a synchronized matrix across the cortical sensory area ${ }^{135}$. Similar investigations are still needed to determine the specific cellular mechanisms of thalamocortical synchrony in mice.

\section{Communication through Coherence (CTC) in Thalamocortical Transmission}

The communication through coherence hypothesis is that neuronal oscillations contribute to sensory processing by modulating the effectiveness of communication among neurons ${ }^{16}$. Modulation of afferent transmission of sensory information would thus help define neuronal representation of that information, as well as the transformations it undergoes along the pathway, leading to computation. Our current understanding of the thalamic role in sensory processing is one of a dedicated relay of salient information, likely with little transformation. If CTC plays a role in thalamocortical communication, one would therefore expect strong phase synchronization within a limited frequency band accompanied by highly effective transmission. By recording LFPs and spikes in dLGN and V1 simultaneously, it was possible to infer the effectiveness of feed-forward neuronal communication and link it to the presence of oscillatory gamma-band activity in V1. During periods of gray screen viewing, synchrony of spike events was exclusively correlated with activity in a narrowband gamma frequency $(50-60 \mathrm{~Hz})$, suggesting a role for this oscillation in feed-forward neuronal communication. This was a predictable result given the previous report that narrowband currents exist in V1 even during optical silencing of local activity ${ }^{121}$. Using the same technique, I further identified oscillations in a broader gamma band $(50-90 \mathrm{~Hz})$ which were strongly correlated with spike synchrony, and driven by visual stimulation with high contrast. Across stimulus conditions, amplitude fluctuations in gamma band activity in V1 were largely attributable to the degree of synchrony with dLGN gamma.

\section{Conclusion}

To the best of our knowledge, our results are the first derived from simultaneous recordings of LFP and spikes in V1 and dLGN in awake mice, which allowed us to directly link phase relationships between oscillations with spike synchrony in both areas. Our data show that in awake mice oscillations in the high gamma rage facilitate feedforward neuronal communication from dLGN to V1, and that stimulus properties have strong modulatory effects on the amplitude, bandwidth, and center frequency of these oscillations. In a broader context, our findings provide additional, strong support for the hypothesis that coherence of neuronal oscillations provides a mechanism for facilitating neuronal communication between brain areas ${ }^{16}$ and that different processing mechanisms may be appropriated to different frequency bands ${ }^{17,19}$. Comparing our findings with those of other groups, species-specific differences in the use of coherent oscillations likely exist 
in form of differences in the functional roles of specific frequency bands ${ }^{18}$, evidence for communication through coherent oscillations was found in all mammalian species investigated thus far ${ }^{13,136-138}$ and thus emerges as a potential general principle for the coordination of neuronal communication. 


\section{CHAPTER 4. CEREBELLAR PURKINJE CELL TUNING TO PHASE DIFFERENCES BETWEEN THE MEDIAL PREFRONTAL CORTEX AND HIPPOCAMPUS IN MICE}

The cerebellum has indirect reciprocal anatomical connections with non-motor areas of cerebral cortex, including the prefrontal cortex and hippocampus. These connections have been implicated in cognitive behaviors but the functional interactions between the cerebellum and non-motor areas are poorly understood. In order to address this question, triple-site simultaneous recordings were performed in the medial prefrontal cortex (mPFC), the dorsal hippocampal CA1 region (CA1) and in either the cerebellar lobulus simplex (LS) or Crus I in awake, head-fixed mice. The mPFC and hippocampus are reciprocally connected and jointly involved in memory functions. Here it is reported that Purkinje cell (PC) simple spike activity in LS and Crus I represents phase differences between oscillations in the mPFC and CA1. The majority of PCs in both cerebellar lobules represented mPFC-CA1 phase differences in more than one frequency band. Between the thirty-two LS PCs analyzed here, phase differences in four conventional frequency bands (delta, theta, beta and gamma) were represented. The majority of Purkinje cells in Crus I represented phase differences in the theta and beta bands. These observations suggest that the cerebellum could play a role in coordinating rhythmic neuronal activity in two cerebral cortical areas, rather than - or in addition to - modulating neuronal activity in a single target structure.

\section{Introduction}

The cerebellum was long perceived as a structure exclusively devoted to coordination of movements, but it is now widely recognized for its involvement in cognition, in both experimental animal models and humans ${ }^{139-142}$. A neuronal mechanism for cerebellar involvement in cognition has not yet been identified. While the performance of cognitive tasks does not require solving the mechanical problems associated with coordinating inertial masses during body and limb movements, precise temporal coordination of neuronal activity is a requirement common to both motor ${ }^{143-146}$ and non-motor tasks ${ }^{147-152}$. Here the hypothesis is assessed that the neuronal mechanisms of cerebellar involvement in cognitive function involves the temporal coordination of neuronal activity by the cerebellum. Specifically, it was investigated whether the temporal relationship of oscillatory phases in the neuronal activity in the medial prefrontal cortex (mPFC) and the hippocampal CA1 region (CA1) is reflected in the timing of cerebellar Purkinje cell (PC) activity.

There is broad consensus that the cerebellum plays a key role in several tasks involving precise timing, including the temporal coordination of movements ${ }^{144,153-159}$, the perception of temporal patterns ${ }^{160-164}$ and predictive timing related to learning ${ }^{165-167}$. A form of precise temporal coordination associated with cognitive function is the taskrelated modulation of coherence of local field potential (LFP) oscillations between two structures $^{14}$. An increase in the coherence of LFP oscillations has been suggested as a 
mechanism to enhance neuronal communication ${ }^{16}$. The mPFC and dorsal CA1 region express increased coherence of LFP theta and gamma band oscillations during the decision-making process in a spatial working memory (SWM) task ${ }^{168,169}$. Adjusting the coherence of oscillations between two structures requires the precise temporal alignment of the phases of oscillations in the two structures. Here it was asked whether the cerebellum is involved in this timing task, targeting specifically the coordination of oscillation phases between the medial prefrontal cortex (mPFC) and the hippocampal CA1 area (CA1).

To address whether the cerebellum might be involved in the temporal coordination of phase relationships between oscillations in two structures that are devoted to non-motor brain functions, simultaneous recordings of LFP oscillations in the $\mathrm{mPFC}$, dorsal CA1 and single unit PC spike activity in cerebellar lobulus simplex were acquired and analyzed. These results show that the phase relationship between oscillations in the $\mathrm{mPFC}$ and CA1 are represented in lobulus simplex and Crus I PC activity. PC phase difference representations were found in delta, theta, beta, low and high gamma frequency oscillations, with different PCs representing different frequency bands. PCs representing phase differences within the theta and low gamma bands stood out, as they all shared a preference for representing phase differences with the mPFC phase leading CA1 phase.

These findings are evidence that the cerebellum is involved in the temporal coordination of phase relationships between oscillations in non-motor brain areas and support a cerebellar role in to the coordination of functional connectivity by modulating coherence.

\section{Methods}

\section{Animals}

Experiments were performed on six adult male C57BL/6J (B6) mice ( $>8$ weeks old, 18-25 g body weight). Male mice were chosen to avoid outcome variability due to menstrual cycle ${ }^{170}$. Mice were housed in a breeding colony with 12-hour light/dark cycles in standard cages with ad libitum access to food and water. All experiments were performed during the light cycle, between 12:00 noon and 17:00 hours. None of the mice had undergone any previous experimental procedure. All animal experimental procedures adhered to guidelines approved by the University of Tennessee Health Science Center Animal Care and Use Committee. Principles of laboratory animal care (NIH publication No. 86-23, rev. 1996) were followed.

\section{Preparation for Head-Fixed Recording}

In preparation for surgery, mice were initially anesthetized in an induction chamber and anesthesia was induced with 3\% Isoflurane in oxygen. Anesthetized mice 
were transferred to a stereotaxic head frame and anesthesia was maintained with 1-2.5\% Isoflurane in oxygen delivered via a nose cone. Isoflurane concentration was controlled with a vaporizer (Highland Medical Equipment, CA), and maintained at the lowest concentration at which mice failed to show reflexive withdrawal to a toe pinch. Core body temperature was measured and maintained at $37^{\circ} \mathrm{C}$ using a feedback-controlled heating pad with a rectal thermometer (FHC Inc., Bowdoinham, ME).

Details of the surgical procedure to prepare for head-fixed recording have been described elsewhere ${ }^{123}$. In brief, scalp fur was removed and the exposed skin treated twice with iodine. The skull was exposed and cleaned and dried with a scalpel and sterile cotton swabs. Two craniotomies were made, one over the left prefrontal cortex and hippocampus, and one over the right cerebellar lobulus simplex. The dura was left intact and covered with triple antibiotic ointment (Walgreens Co., US) to maintain moisture and prevent infection. A cylindrical plastic chamber was placed on each craniotomy for mechanical protection. Three small screws were implanted into the skull to serve as anchors for acrylic cement. A head fixation bar was placed onto the skull and held in place with a drop of cyanoacrylate glue. Finally, acrylic cement (Co-Oral-Ite Manufacturing, New Franken, WI) was applied to the skull screws, the plastic chambers and head fixation bar and allowed to cure. Mice were injected with Carprofen $(5 \mathrm{mg} / \mathrm{kg})$ for analgesia once at the end of surgery before discontinuing anesthesia and again the morning of the next day. Mice were allowed to recover from surgery for 3 to 4 days prior to recording.

\section{Electrophysiological Recordings}

During recordings mice were standing on a flat surface, their bodies gently covered with a loosely fitting $1 / 2$ tube and the head fixation bar was secured to two stationary metal arms using small machine screws. Mice were allowed to adapt to head fixation for at least fifteen minutes prior to recording. Once the mice were adapted, the recording chambers were rinsed and filled with sterile saline.

A single 7-electrode micromanipulator and headstage (System Eckhorn; Thomas Recording, Germany) was used to record simultaneously from the three brain areas. This system allows micrometer precise control of the penetration depth of each electrode individually. A custom made set of guiding tubes was prepared to guide electrodes into each area. Guiding tubes were lowered into the plastic chambers so that their tips were just above the dura. The extracellular recording electrodes (glass-insulated tungsten/platinum; $80 \mu \mathrm{m}$ O.D.; impedance, 3-7 M) were then slowly advanced through the dura to their target location. Hippocampal recording electrodes were positioned such that sharp wave ripples (SWRs), a brief high-frequency local field potential (LFP) oscillation characteristic for the hippocampus ${ }^{171}$, could be clearly identified (Figure 4-1). Cerebellar recording electrodes were advanced into the lobulus simplex, until a single unit spike train with characteristic complex spikes, which identify Purkinje cells was isolated. Recording locations in the prefrontal cortex were selected following stereotaxic coordinates relative to Bregma ${ }^{172}$ and were verified anatomically using small electrolytic 

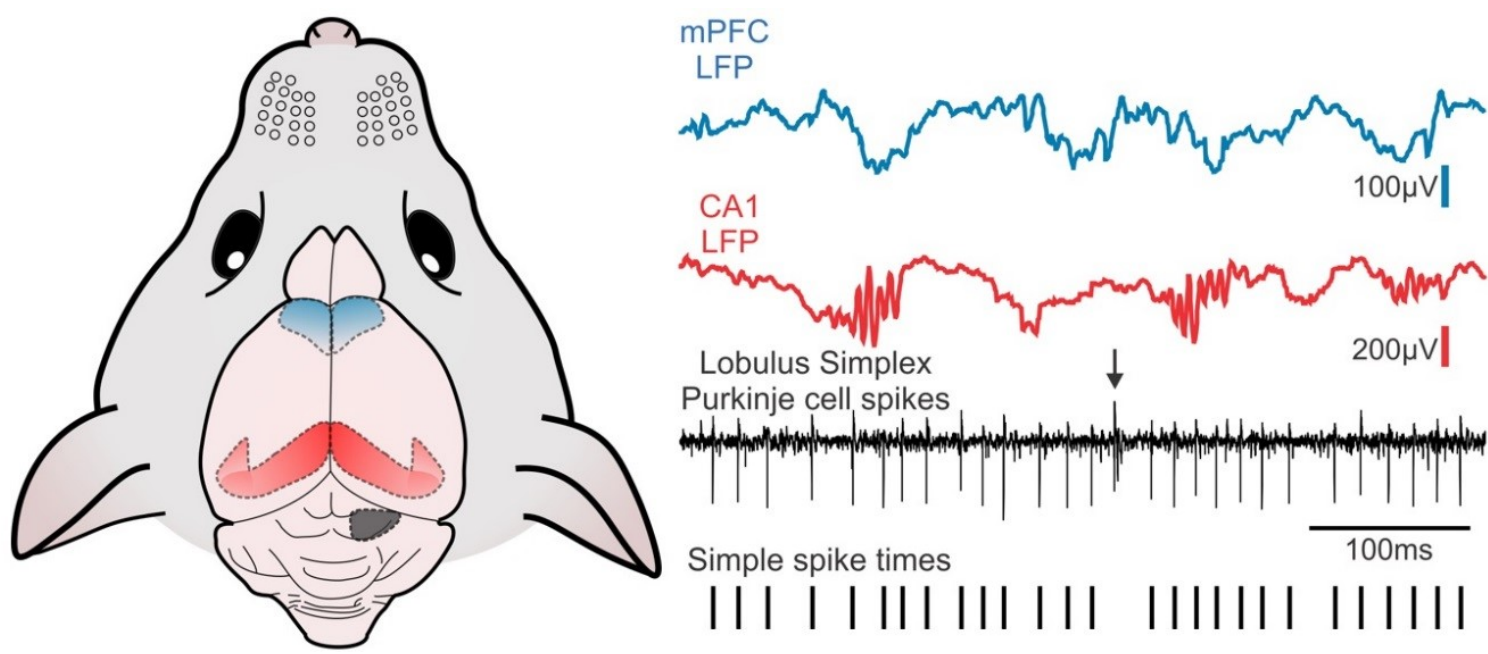

Figure 4-1. Composite illustration of raw LFP signals and single unit PC spike activity recorded in the $\mathrm{mPFC}, \mathrm{CA} 1$ and cerebellar lobulus simplex.

The LFP recording from the CA1 region reveals sharp wave ripple events, which are brief high-frequency oscillations characteristic for the hippocampus. The presence of sharp wave ripples in the LFP recordings was used for online verification of electrode tip placement within CA1. In the trace of raw PC spike activity a black arrow marks the occurrence of a complex spike, which are characteristic for PCs and were used for online identification of PC activity. The bottom trace shows tick marks representing the time sequence of simple spikes extracted from the raw trace and used for subsequent analysis. 
lesions created at the end of the final recording session (left mPFC: lat. $0.5 \mathrm{~mm}$, ant. 2.8 $\mathrm{mm}$; left CA1: lat. $2.0 \mathrm{~mm}$, ant. $-2.3 \mathrm{~mm}$; Cbl. lobulus simplex: lat. $2.0 \mathrm{~mm}$, ant. -5.52 $\mathrm{mm})$.

Recordings were repeated three times on three successive days for each mouse. After completion of each recording session, the chambers were rinsed and re-filled with triple antibiotic ointment and mice were returned to their home cages. At the end of the last recording sessions electrolytic lesions were created by passing a small current ( 5 $\mu \mathrm{A} / 10 \mathrm{~s}$ ) through the recording electrodes. Within 12 hours of creating the lesion, mice were euthanized and then transcardially perfused. After perfusion, the brain was removed and post-fixed in $4 \%$ formaldehyde for $24 \mathrm{~h}$. Brain tissue was cut into $50 \mu \mathrm{m}$ thick coronal sections, which were mounted and stained with cresyl violet. The location of lesion sites was determined using a stereotaxic atlas of the mouse brain ${ }^{172}$.

LFPs and spike signals were separated by band-pass filtering at 0.1 to $200 \mathrm{~Hz}$ and at $200 \mathrm{~Hz}$ to $8 \mathrm{kHz}$, respectively, using a hardware filter amplifier (FA32; Multi Channel Systems, Germany). Filtered and amplified voltage signals were digitized and stored on a computer hard disk (16 bit A/D converter; sampling rate, $>20 \mathrm{kHz}$ for action potentials, $>1 \mathrm{kHz}$ for LFPs) using a CED power1401 and Spike2 software (both Cambridge Electronic Design). This study focused on the relationship between single-unit spike activity in cerebellar PCs and LFP oscillations in the PFC and CA1. After stable LFP recordings were established in PFC and CA1 no further attempt was made to isolate single unit spike activity in the two areas.

\section{Analysis of PC Single-Unit Spike Data}

Single-unit spike activity was recorded from 32 PCs located within cerebellar lobulus simplex in 6 mice. PCs were identified based on their location and firing characteristics such as the presence of complex spikes as well as sustained highfrequency simple-spike firing rates ${ }^{173}$. Simple spike activity was identified off-line using a shape-based spike-sorting algorithm in the Spike2 software. In our sample of 32 PCs simple spike firing rates ranged from 36.5 to $157.9 \mathrm{~Hz}$, with a mean of $89.1 \mathrm{~Hz}$. Complex spikes were excluded from the analysis. Because of their expected low frequency $(\sim 1$ $\mathrm{Hz}$ ), the number of complex spikes in our data samples was too low for meaningful statistical evaluation of their relationship with mPFC-CA1 with phase differences.

\section{Pre-Preprocessing of LFPs}

Only sections of data with stable single-unit spike isolation and LFP recordings, which were free of movement artifacts, were included in analysis. CA1 recording sites were included only if they showed clear sharp wave ripples (Figure 4-1, CA1 LFP). $60 \mathrm{~Hz}$ noise was diminished using a hum-removal algorithm in Spike2 that subtracted $60 \mathrm{~Hz}$ components rather than applying a notch-filter, which left the power spectrum of LFP signals intact. 


\section{Estimation of LFP Phase Relationships and Corresponding Spikes}

In order to determine phase relationships of LFP oscillations in PFC and CA1 over time, LFPs were band-pass filtered for frequencies $0.5-100 \mathrm{~Hz}$. FIR filters were applied in frequency steps of $0.25 \mathrm{~Hz}$ with bandwidth ranging from $0.5-10 \mathrm{~Hz}$ with increasing frequency. Filter order was determined to be the number of samples within 5 cycles of the center frequency. The Hilbert transform was performed on each band-passfiltered signal, and instantaneous phase values were defined as the angular value derived from the complex-valued analytic signal. The phase relationship between PFC and CA1 oscillations within each frequency band was determined in terms of the difference in instantaneous phase values, with $\mathrm{mPFC}$ phase being subtracted from CA1 phase. The phase relationships were then designated into 30 angular bins of $12^{\circ}$ width, and the corresponding spikes within each bin were counted. The spike count in each bin was divided by the total number of angular samples in the bin to return a measure of spike density (Figure 4-2C). Each bin was then treated as a vector with an angular value of the phase relationship and magnitude of the spike density in order to calculate a resultant vector with magnitude reflecting degree of modulation and direction of preferred phase (Figure 4-2D).

\section{Statistical Analysis}

Bootstrap statistical analysis of the relationship between spike activity and PFCCA1 phase differences was performed by calculating vector lengths for 200 surrogate data sets. Each surrogate data set was created by random, circular shifting of the spike sequence. The resulting 200 surrogate vector values were rank-ordered for each frequency and the 95th and 99th percentile values were determined. Real vector values greater than the 95th percentile of the surrogate vector distribution were considered statistically significant, and those greater than the 99th percentile were selected for further group analysis.

\section{Results}

\section{Link between PFC-CA1 Phase Differences and Spike Rate in Individual PCs}

Recordings of LFP activities in the MPFC and dorsal CA1 region were obtained while simultaneously recording PC simple spike activity in the cerebellar lobulus simplex (Figure 4-1) and Crus I. All recordings were performed in awake, head-fixed mice at rest. In order to determine whether the simple spike activity of PCs in LS is correlated with phase differences between LFP oscillations in the MPFC and CA1, PC spike activity was quantified as a function of phase difference between oscillations in the MPFC and CA1 for frequencies between 0.5 and $100 \mathrm{~Hz}$. A conceptual illustration of the data analysis process is provided in Chapter 2 (Figure 2-9). The relationship between spike rate and phase difference was quantified for each frequency band by calculating a vector 


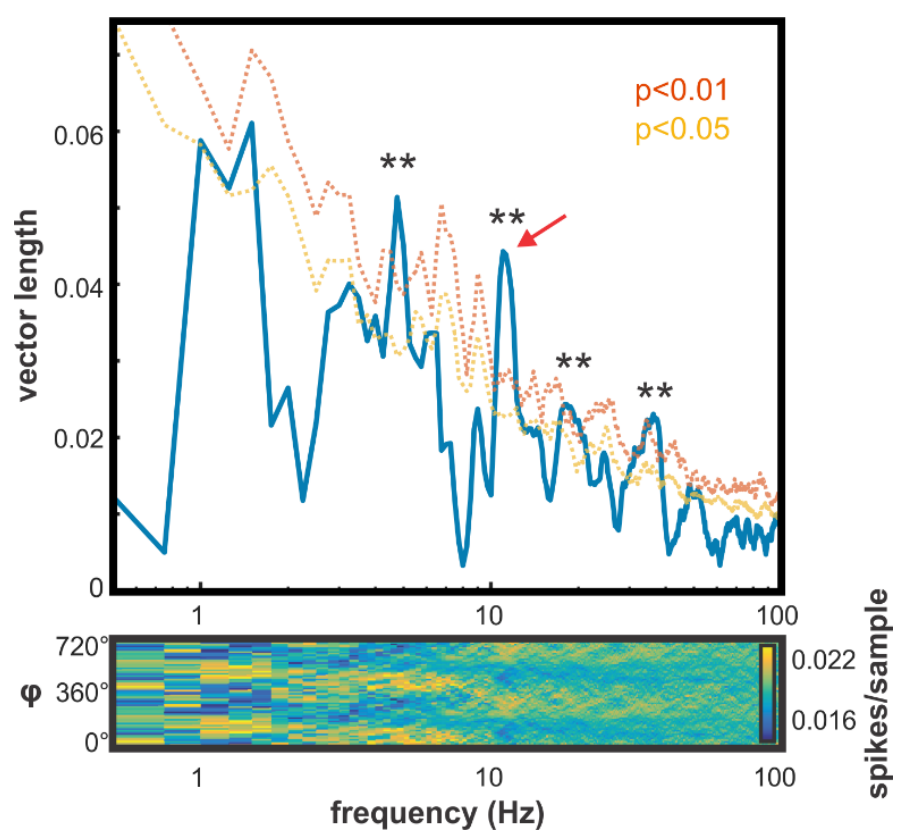

Figure 4-2. Example analysis for one $P C$ showing how phase differences across the full frequency spectrum of LFP oscillations $(0-100 \mathrm{~Hz})$ are represented in this cell's simple spike activity.

Blue line shows depth of PC spike modulation, as measured by the resultant vector magnitude (cf. Figure 2-9C and D). Significance cutoffs represent the $95 \%$ ile and $99 \%$ ile boundaries, respectively, of the surrogate result distributions for each frequency obtained by shifting the PC spike recording in time relative to LFPs 200 times. Asterisks indicate frequencies with significant modulation $(\mathrm{p}<0.01)$. Red arrow indicates the frequency analyzed to create the histogram and polar plot shown in Figure 2-9C and D.

Pseudocolor plot shows PC spike density as a function of phase difference $(\varphi)$ between mPFC and CA1 LFP oscillations across frequencies between 0.5 and $100 \mathrm{~Hz}$. Analysis was performed within discrete frequency bands of $0.25 \mathrm{~Hz}$ width. 
with direction corresponding to a preferred $\mathrm{mPFC}-\mathrm{CA} 1$ phase difference and length representing how strongly spike activity reflected that particular phase difference (Figure 4-2). Statistical significance of spike modulation as a function of mPFC-CA1 phase difference was determined using a Monte Carlo hypothesis test (see Methods section for details). Significant firing rate modulation with $\mathrm{mPFC}-\mathrm{CA} 1$ phase differences was found in 29 out of $32 \mathrm{PCs}(90.6 \%)$ in LS and in 14 of 17 PCs (82.35\%) in Crus I.

\section{PC Activity Modulation Occurs across the Frequency Spectrum of PFC/CA1 Oscillations}

In an analysis of group data it was determined what fraction of PCs showed significant phase-related rate modulations at each analyzed frequency (Figure 4-3). The results suggest that there are clear "preferred" frequencies, whose phase differences are represented by large proportions of observed PCs, while other frequencies are not represented at all. The analysis of LS data revealed that spike rates were most commonly modulated by phase differences in the theta band, with the highest number of PCs representing phase differences for $5 \mathrm{~Hz}$ oscillations (Fig. 4C). Significant results also clustered into modes within each of the other conventional frequency bands: delta $(2 \mathrm{~Hz})$, beta $(17.5 \mathrm{~Hz})$, low gamma $(35 \mathrm{~Hz})$ and high gamma $(55 \mathrm{~Hz})$.

PCs in Crus I also represented phase differences only for specific mPFC and CA1 oscillations, with different preferred frequencies, compared to LS PCs (Fig. 4A,E). PC activity in Crus I was also most commonly modulated in the theta band, but with slightly higher frequency $(8 \mathrm{~Hz})$. Other modes were observed at $2.5 \mathrm{~Hz}$ (delta), $15.25 \mathrm{~Hz}$ (beta), $45 \mathrm{~Hz}$ (low gamma).

\section{Discussion}

Here it is reported that simple spike activity of LS and Crus I PCs in awake, headfixed mice represents phase differences between LFP oscillations in the MPFC and CA1. Individual PCs in both cerebellar cortical areas represented phase differences between $\mathrm{mPFC}$ and CA1 oscillations in one or more of the conventional frequency bands. However, phase differences are not represented equally for all frequencies. There clearly are preferred frequencies whose phase differences are represented by a large proportion of the recorded PCs, which is reflected in distinct peaks in the graph showing fractions of PCs representing phase differences vs. frequency (Figure 4-3). In the LS, those preferred frequencies were $2 \mathrm{~Hz}$ (delta), $5 \mathrm{~Hz}$ (theta), $17.5 \mathrm{~Hz}$ (beta), $35 \mathrm{~Hz}$ (low gamma) and $55 \mathrm{~Hz}$ (high gamma). In Crus I, the preferred frequencies were $2.5 \mathrm{~Hz}$ (delta), $8 \mathrm{~Hz}$ (theta), 15.5 $\mathrm{Hz}$ (beta), $45 \mathrm{~Hz}$ (low gamma). In addition, in Crus I three PCs represented phase differences in high gamma, but each cell for a different frequency. The most prominent difference that distinguishes the activity of PCs in the LS compared to Crus I is the much smaller portion of PCs representing delta band phase differences, with $28 \%$ of PCs in LS vs. $11.7 \%$ in Crus I. 

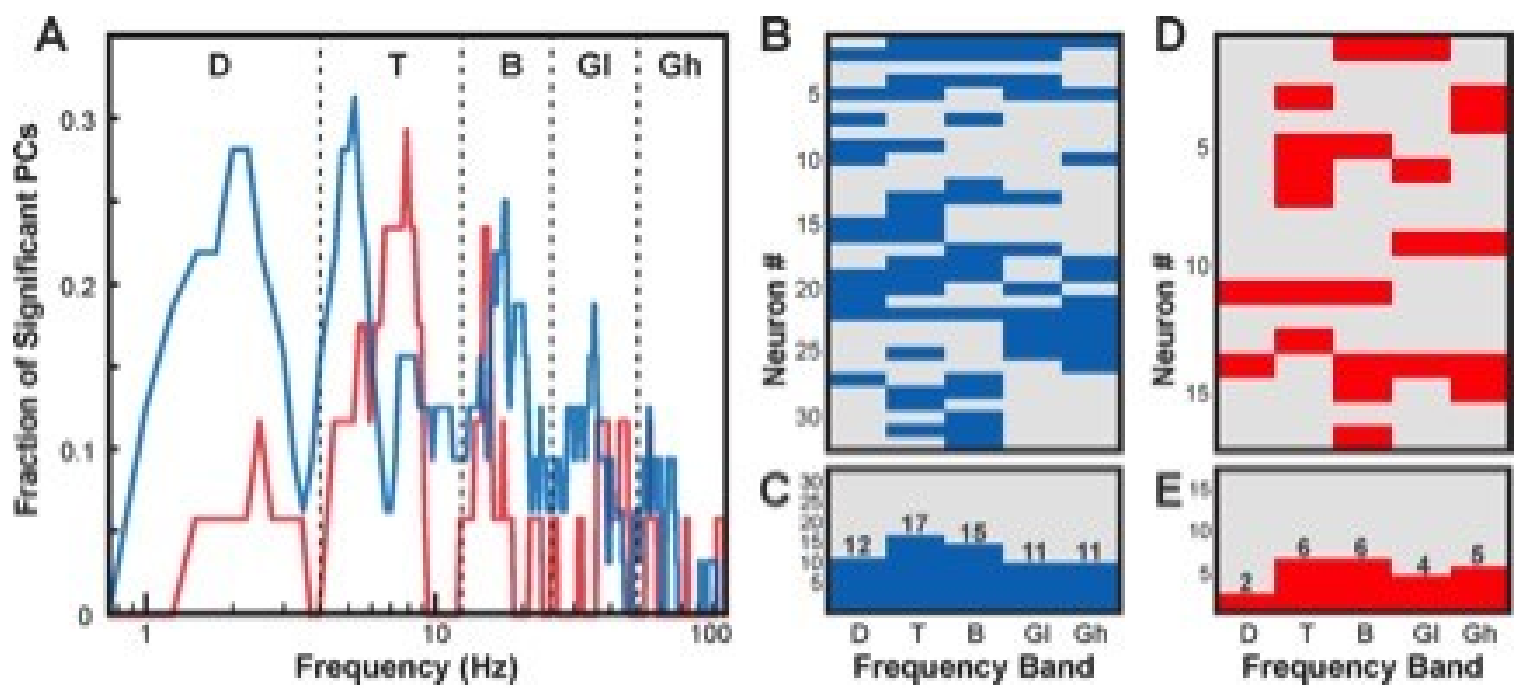

Figure 4-3. Summary of group results across all analyzed Purkinje Cells recorded in LS $(n=32)$ and Crus I $(n=17)$.

(A) Plot depicting the fraction of PCs with significantly modulated simple spike activity $(\mathrm{p}<0.01)$ within each $0.25 \mathrm{~Hz}$ frequency step between 0.5 and $100 \mathrm{~Hz}$ in LS (blue graph) and Crus I (red graph). Modal peaks were observed within each of the conventional frequency bands: Delta (D; 0.5-4Hz), Theta (T; 4-12Hz), Beta (B; 12-25Hz), Low Gamma (GL; 25-45Hz), and High Gamma (GH; 45-100Hz). (B) Pseudocolor matrix with rows representing individual PCs and columns representing frequency bands. Blue fields indicate a significant correlation between the simple spike activity of the individual PC in LS and phase differences between mPFC-CA1 oscillations in each specific frequency band $(p<0.01)$. Grey fields indicate no significant correlation. (C) Histogram showing the number of PCs with significant spike-phase difference correlations within each frequency band. (D) and (E) are as (B) and (C) but representing results from Crus I. 
How cerebellar PCs come to represent phase differences between mPFC and CA1 oscillations remains to be determined. Considering that any phase difference for a given frequency can be represented as a time interval, a possible neuronal mechanism can be derived from the unique cerebellar network architecture, with parallel fibers serving as delay lines transforming phase-difference encoding delays between mossy fiber spike events into synchronous inputs to PCs ${ }^{174-176}$.

While the behavioral and cognitive significance of our findings remains to be determined, it is worthwhile to consider that the cerebellum, $\mathrm{MPFC}$ and CA1 are jointly implicated in spatial processing and SWM ${ }^{177,178}$ and that the process of decision making in working memory involves a temporary stabilization of phase differences (i.e. increased coherence) between mPFC-CA1 oscillations. ${ }^{169}$. How the cerebellum contributes to SWM is not understood, but our results suggest a cerebellar role in modulating mPFCCA1 coherence, which is concordant with finding that normal coherence between sensory and motor cortical areas requires an intact cerebellum ${ }^{179}$. Coherence has been proposed a mechanism for the coordination of neuronal communication ${ }^{16,87}$. Our analysis provides evidence of cerebellar involvement in the temporal coordination of oscillatory phase relationships in remote brain regions, a function that would be in line with the longstanding notions of cerebellar timing and temporal coordination functions ${ }^{180,181}$. 


\section{CHAPTER 5. RESPIRATION AS A FUNDAMENTAL RHYTHM OF BRAIN FUNCTION}

\section{Introduction}

Oscillatory patterns of activity within sensory cortical regions appear to critically shape the effectiveness of transmission between the neurons within, causing transformations in the neuronal representation of the sensory environment as perception takes place. Thus, it is important to understand how oscillations are generated, as well as what factors lead to their emergence and influence their characteristics.

One factor that appears to work as both a driver and modulator of neuronal oscillations in sensory regions is the respiratory rhythm. In their first published example, Ito et al. demonstrated that the whisker sensory cortex of the mouse exhibited prominent delta oscillations that were time-locked to breathing, as well as high amplitude gamma oscillations that were phase-aligned with the beginning of expiration ${ }^{90}$. Importantly, they also showed that this link between neuronal rhythms and respiration was abolished when the olfactory bulb (OB) was surgically removed.

The hypothetical importance of these rhythms for the processing of tactile input from the whiskers is fairly straightforward, as the rhythms of whisking and breathing are synchronized in rodents. Perhaps by synchronizing time windows of high excitability in the cortex with sensory transduction at the receptor level, the whisker cortex can become most receptive when it is most likely to receive information, or even perform phase coding for stimulus strength by adding this oscillatory element to its network activity. However, two things remain curious about this finding if these hypotheses are to be accepted at face value. First, it is extraordinary for the predominant portion of the neuronal activity within a primary sensory region to be driven by activity related to an entirely different sensory modality. Post-bulbectomy LFP recordings from the whisker sensory cortex showed little fluctuation, revealing that afferent whisker input wasn't sufficient to drive normal levels of oscillatory activity in the whisker sensory cortex. Secondly, it was later reported that respiration-locked LFP oscillations were not constrained to sensory regions that process input in a phasic manner like whisking, as the visual and prefrontal cortex also exhibited these oscillations (Figure 5-1).

So what is the role of respiratory-locked activity in the brain? To answer this question we must first understand how respiration drives neuronal rhythms, both in terms of degree or amplitude and in terms of cross-regional breadth. In the case of whisker sensory cortex, respiration-locked activity is driven by oscillations in the olfactory bulb. It was known as long ago as 1942, when Lord Adrian recorded from the OB of the hedgehog ${ }^{182}$, that the OB generates electrical activity in synchrony with respiration even in the absence of odor. These oscillations, as well as concurrent high frequency beta and gamma oscillations, are generated by sensory stimulation from nasal airflow ${ }^{183}$. These oscillations are often associated with early odor processing that occurs in the bulb ${ }^{52,184,185}$, and necessarily drive phasic excitatory output from the bulb even in the absence of 
Figure 5-1. Respiration-locked oscillations and spikes throughout the olfactory bulb and neocortex.

Blue traces at the top of each panel show LFP averages aligned on the peaks of the respiratory cycle, shown as the dashed green line. Bottom blue traces show correlations between spike rate and respiration. Red lines show 99\% confidence intervals for statistical significance as determined by 1000 random time shifts. Box at bottom left shows example of single unit isolation and inter-spike interval for single unit identification. Olfactory bulb-dependent cortical espiration-locked oscillations were first identified in the whisker sensory cortex (Ito et al. 2014), but since then have been identified throughout the cortex. Examples presented here illustrate the strong presence of these oscillations in the prefrontal cortex, whisker motor cortex, somatosensory cortex for the trunk region, and the primary visual cortex. Spike rate modulation corresponds strongly to respiration-locked deflections in the LFP, strongly suggesting these oscillations influence ongoing patterns of neuronal activity related to sensory and cognitive processing, rather than an artifact of movement.

odor ${ }^{183,186}$. For mammals such as rodents where the olfactory bulb constitutes a large proportion of the brain mass, these respiration-locked outputs are a substantial excitatory barrage occurring with every breath. 


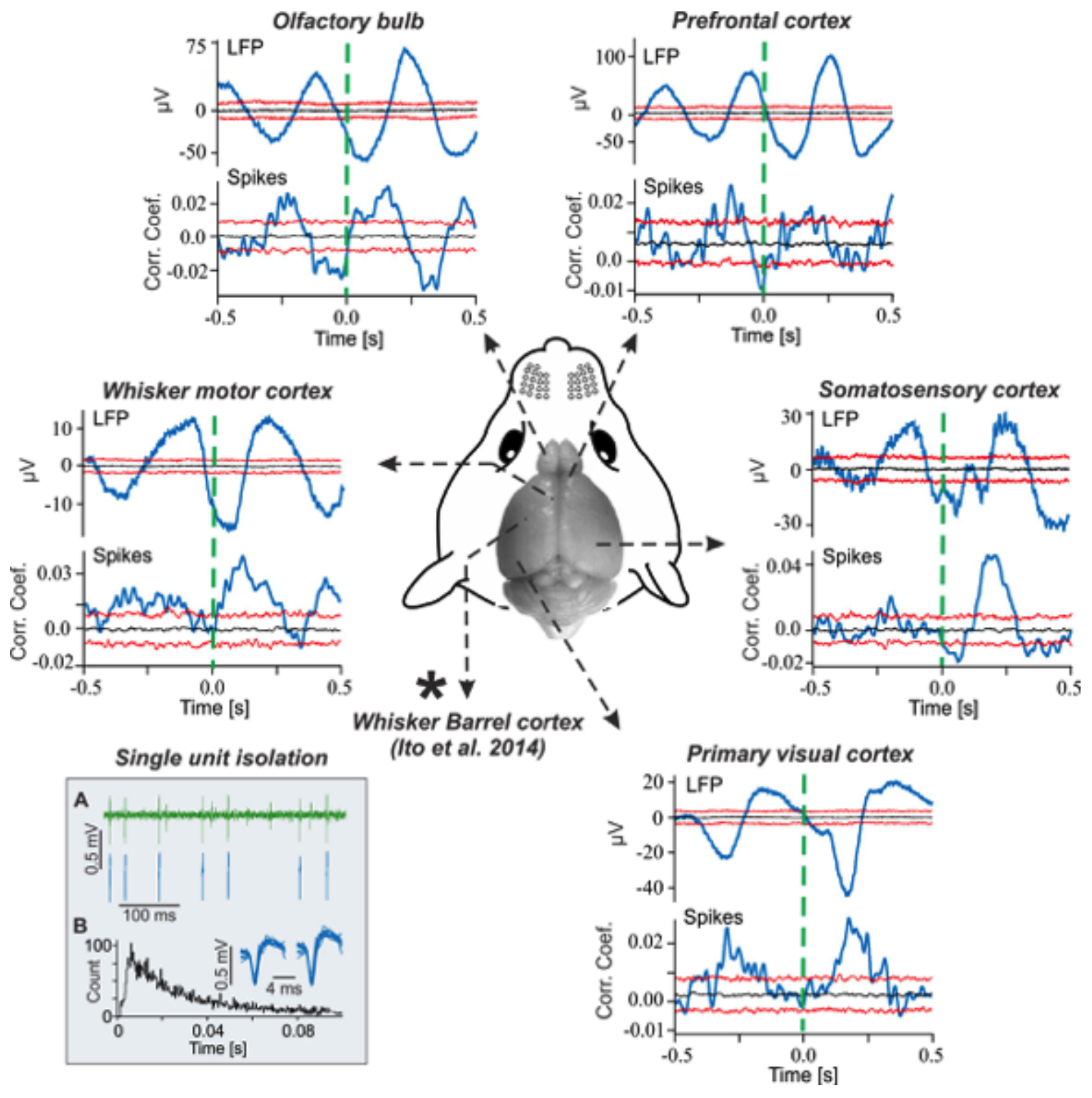


This output directly excites the piriform cortex, which is known to oscillate synchronously with many regions, such as the basal forebrain ${ }^{187}$, ventral striatum ${ }^{188}$, hippocampus ${ }^{189,190}$, and prefrontal cortex ${ }^{191}$. Each of these regions plays a crucial role in normal cognitive and behavioral function. More specifically, the basal forebrain contains cholinergic neurons which project throughout the neocortex and modulate global brain states, as well as long-range inhibitory neurons that disinhibit sensory thalamic relay neurons ${ }^{192}$. The ventral striatum is critically involved in reward-seeking behavior and motivation $^{193}$. The prefrontal cortex is viewed as the hub of higher cognitive operations, where sensory inputs from all modalities are integrated with input from the hippocampus and other limbic structures ${ }^{194-196}$. Given the degree of rhythmic excitation to these crucial regions and the extent of their connections to other areas, it seems likely that the rhythm of respiration plays a role in the cognitive, emotional, and sensory processes that they carry out.

This has become the foundation of the hypothesis that breathing works as a fundamental rhythm of brain function ${ }^{197}$. Since the respiratory rhythm is a phylogenetically ancient function, it seems likely that the evolutionary development of other sensory and cognitive systems occurred in the presence of strong respiratory modulation, leaving open the possibility that even primate and human brains operate with some dependence on this essential rhythm.

In order to understand the possible functional role of respiration beyond supplying the body with oxygen, it is essential to identify and fully characterize the extent to which brain activity is temporally structured around this rhythm. Here I will present a suite of findings which link distributed patterns of brain activity to the rhythm of respiration both in rodents and humans, including the occurrence of time-locked slow rhythms, the phaselocked modulation of fast rhythms, and the entrainment of hippocampal activity related to memory. In order to do this effectively in mice, it is necessary to use an effective method of measurement for the respiratory rhythm, thus my recently developed method will first be described.

\section{Development of an Effective Method for Measurement of Respiration in Mice*}

\section{Overview}

Background. Respiration is one of the essential rhythms of life. The precise measurement of respiratory behavior is of great importance in studies addressing

\footnotetext{
* Modified with permission of ELSEVIER BV in the format Thesis/Dissertation via Copyright Clearance Center. McAfee, S.S., Ogg, M.C., Ross, J.M., Liu, Y., Fletcher, M.L., Heck, D.H. Minimally invasive highly precise monitoring of respiratory rhythm in the mouse using an epithelial temperature probe. J Neurosci Methods 263, 89-94, doi:10.1016/j.jneumeth.2016.02.007 (2016) ${ }^{198}$.
} 
olfactory sensory processing or the coordination of orofacial movements with respiration. An ideal method of measurement should reliably capture the distinct phases of respiration without interfering with behavior.

New Method. This new method involves chronic implantation of a thermistor probe in a previously undescribed hollow space located above the anterior portion of the nasal cavity without penetrating any soft epithelial tissues.

Results. The reliability and precision of the method is demonstrated in head-fixed and freely moving mice by directly comparing recorded signals with simultaneous measurements of chest movements and plethysmographic measurements of respiration.

Comparison with Existing Methods. Current methods have drawbacks in that they are either inaccurate or require invasive placement of temperature or pressure sensors into the sensitive nasal cavity, where they interfere with airflow and cause irritation and damage to the nasal epithelium. Furthermore, surgical placement within the posterior nasal cavity adjacent to the nasal epithelium requires extensive recovery time, which is not necessary with the described method.

Conclusions. A new method is described for recording the rhythm of respiration in awake mice with high precision, without damaging or irritating the nasal epithelium. This method will be effective for measurement of respiration during experiments requiring free movement, as well as those involving imaging or electrophysiology.

\section{Introduction}

Respiration, a fundamental rhythm of mammalian life, is inextricably linked to olfaction $^{52,199}$ and sensory system activity ${ }^{90,200}$ in rodents, but poses unique challenges in terms of accurate measurement. Current methods employ the use of temperature or pressure sensors placed within the nasal cavity ${ }^{199,201,202}$ or in front of the nares ${ }^{90}$. However, these methods have significant drawbacks.

Extranasal thermistors are an easy-to-use, non-invasive option for capturing the temperature changes associated with respiration for use in head-fixed mice. However, extranasal thermistors become less reliable during periods of high-frequency sniffing. This is because mice can move their nares and thereby redirect the respiratory airstream, which they often do during sniffing. This causes variability in the phase of the readout and can temporarily result in the loss of signal for one or more cycles. The beginning of inspiration is also difficult to reliably capture, as the probe is continually cooling towards the room temperature. Consequently, extranasal thermistors are most effective when 
expiration onset during resting breathing $(<6 \mathrm{~Hz})$ is of interest, and can be used effectively for creating angular data ${ }^{203}$.

Pressure sensor coupled chambers for plethysmography offer highly precise measurement of respiration ${ }^{204}$, but, by their nature, require rather small recording chambers. This makes them impractical for behavioral experiments that require space for equipment and allow some degree of free movement for the animals. However, simple odor response tests have been successfully conducted in mice ${ }^{205}$ and rats ${ }^{206}$ with this approach.

Chronic implantation of sensors allows for the greatest potential mobility for the animal, but current approaches typically penetrate the bone and epithelial tissue overlying the nasal cavity ${ }^{199,201,202}$. Pressure changes associated with respiration are measured by placement of a small cannulae into the nasal cavity, allowing for highly accurate readings of respiratory efforts. However, the placement of even very small cannulae into the cavity will cause partial blockage of the narrow airway, leading to irritation and the formation of mucus. Cannulae are prone to blockage by this mucus or blood over time, requiring the blockage to be cleared using high pressure, suction, or the insertion of a wire. These procedures are likely to cause irritation of the highly sensitive soft tissues of the nasal cavity and constitute additional stressors for the mouse. In addition, a pressure transducer or hose needs to be mounted to the cannula during readings, which adds considerable weight and mechanical stress above the nasal bridge.

Intranasal temperature sensors as described in some studies ${ }^{207,208}$ suffer similar drawbacks, as they also penetrate the nasal cavity thus interfering with airflow and causing damage to the epithelium. This approach may still be preferable to pressure cannula since connection to a signal-transducing amplifying system can be accomplished with minimal-weight 2-pin connectors mounted on top of the skull rather than a pressure transducer attached to the cannula on the bridge of the nose. An additional advantage is that the 2-pin connectors do not restrict the animal's field of vision and are solidly fixed to multiple anchoring screws placed in the skull bone.

Problems with implanted instrumentation penetrating the nasal cavity are exacerbated in mice due to the narrowness of their nasal cavity and the weakness of their neck muscles, which struggle to support heavy monitoring equipment and connectors. Yet, use of mice for such experiments is highly desirable because of readily available genetic tools and disease models in the species. The method of using a non-invasive implant that is presented here overcomes many of the pitfalls described above. Animals who underwent this implantation rapidly recovered. There is virtually no waiting time, other than recovery from surgery, before high quality respiratory data can be recorded. This is in stark contrast to some current methods, which require days or weeks of recovery before respiratory signals can be recorded ${ }^{208}$. It is demonstrated that this method is highly effective and precise in both head-fixed and freely moving experimental preparations. 


\section{Materials and Methods}

Animals. Adult male and female C57BL/6J mice were housed with food and water ad libitum in a 12:12-h light/dark cycle. All mice used in this study were raised and all experiments were performed in accordance with procedural guidelines approved by the University of Tennessee Health Science Center Animal Care and Use Committee. Principles of laboratory animal care (NIH publication No. 86-23, rev. 1996) were followed. Animals were monitored for up to 4 days post-surgery to ensure proper recovery before experimentation.

Probe Fabrication. Fast time response NTS thermistors (MEASG22K7MCD419, Measurement Specialties; Hampton, VA) were used as the temperature sensitive element of the probe. Leads were cut to $5 \mathrm{~cm}$ in length before wires were separated and stripped of insulation on $1 \mathrm{~mm}$ of the distal end. The stripped ends were then soldered to the pins of a miniature male-female header plug (e.g. Mill-Max Engineering, U.S.A., part\#: 861-13-0XX-10-002000) and the soldered connections were insulated with a thin layer of acrylic cement or epoxy. All probes were tested and treated with $90 \%$ ethanol for disinfection prior to implantation.

Surgical Procedure. Prior to surgery, mice were weighed and injected with a ketamine/xylazine cocktail to induce anesthesia. Maintenance of anesthesia throughout the surgery was accomplished by supplemental injections of ketamine. An analgesic was subsequently given $(0.05 \mathrm{ml}$ carprofen solution, s.c. $)$ to facilitate recovery. Once anesthetized, mice were mounted in a surgical stereotaxic frame using $60^{\circ}$ non-rupture ear bars (David Kopf Instruments, Tujunga, CA). Body temperature was monitored with an electronic rectal thermometer and maintained at $37-38.0^{\circ} \mathrm{C}$ using a feedback controlled heating pad (FHC Inc., Bowdoinham, ME). Eye moisture was maintained by application of sterile lubricating eye ointment (Systane, Alcon Inc., Missuaga, Ontario). With care to avoid contact with the eyes, hair was removed from the scalp and nose with scissors and a depilatory cream and the skin was treated with iodine solution (Xenodine, Veterinary Products Laboratory, Phoenix, AZ).

A sterile scalpel was used to make a midline incision to expose the skull surface from the interparietal skull plate to the anterior edge of the nasal bone. The surface was then cleaned using a dental scraper and sterile cotton swabs. A dental drill $(0.7 \mathrm{~mm}$ round burr) was used to create a hole in the posterior skull for a small machine screw, used to anchor the headcap (B000FN0J58, Antrin Miniature Specialties, Fallbrook, CA). Antibiotic ointment was immediately applied to the screw hole to prevent infection and dryness. The dental drill was also used to create the opening for thermistor implantation above the anterior portion of the nasal bone, centered $0.5 \mathrm{~mm}$ lateral from the midline suture (Nasal fissure $+3.1 \mathrm{~mm}$ ). This typically caused minimal to no bleeding and revealed a small cavity overlying highly vascularized soft tissue. To the best of our knowledge, this cavity has not been previously described in the anatomical literature, but 
exists between the nasal epithelium of the dorsal meatus and the frontonasal bone due to a lack of connective tissues (Figure 5-2). The probe was then carefully lowered into this cavity with forceps, while the connector remained attached to a stereotaxic manipulator. We did not find it necessary to fully insert the probe into the cavity; partial insertion (as seen in Figure 5-1) yielded no appreciable reduction in signal quality over completely inserted probes (data not shown). Partial insertion thus was preferred to further reduce the risk of damage to the nasal epithelium or narrowing of the airway. A small amount of KwikSil epoxy (World Precision Instruments, Sarasota, FL) was then applied to cover the probe and allowed to dry in order to protect the temperature sensitive element and seal the opening. This step was found to be crucial, as direct contact of the probe with acrylic cement seemed to damage the thermistors. Once dry, skull screws were implanted in their pre-drilled holes and a head fixation bar was attached to the skull bone with super glue. The probe connector was then released from the stereotaxic arm and excess lead wire was coiled around the stem of a cotton swab until the connector piece was able to rest adjacent to the skull screws with an orientation that would not interfere with later head fixation. Acrylic dental cement was then applied to the exposed skull, embedding skull screws, thermistor connector and head fixation bar. Mice were then placed in individual cages under infrared heat lamp until fully awake, and monitored for up to 4 days to ensure proper surgical recovery.

Data Acquisition. Recording sessions began on the fourth day after surgery. Data were stored on a computer hard disk ( 16 bit A/D converter, sampling rate $2 \mathrm{kHz}$ ) using a CED Power1401 and Spike2 software (both Cambridge Electronic Design, UK).

Head-Fixed Recording. Accuracy of respiratory monitoring by the implanted thermistor was tested by simultaneously recording respiration with a piezoelectric transducer. Mice were placed into the head fixation apparatus and covered by a foamlined plastic half-tube to prevent injury from excessive movement ${ }^{123}$. The implanted thermistor was connected to a custom-built amplifier and the resulting temperaturerelated voltage changes were digitized at $2 \mathrm{kHz}$ (CED 1401, Cambridge Electronic Design, UK) and stored on hard disk. A piezoelectric strip was placed against the lateral chest wall to detect inflation of the chest cavity. Movements of the chest wall would bend the piezoelectric strip, which resulted in a measurable electrical current (Figure 5-3). A second thermistor connected to a second amplifier was placed in front of the nares for simultaneous comparative recording.

Freely-Moving Recording. Feasibility of recording respiration in freely moving mice with the minimally invasive thermistor was assessed during a recording session in a custom plethysomgraphy chamber (Figure 5-4). Prior to recording the chamber was connected to a pressurized air inlet and pressure was calibrated to create $0.5 \mathrm{~L} / \mathrm{min}$ of continuous airflow through the chamber. The plethysmography chamber ceiling was coupled to the positive inlet of a differential pressure transducer with carrier demodulator (DP45-14 and CD15 respectively, Validyne Engineering, Northridge, CA) to detect 


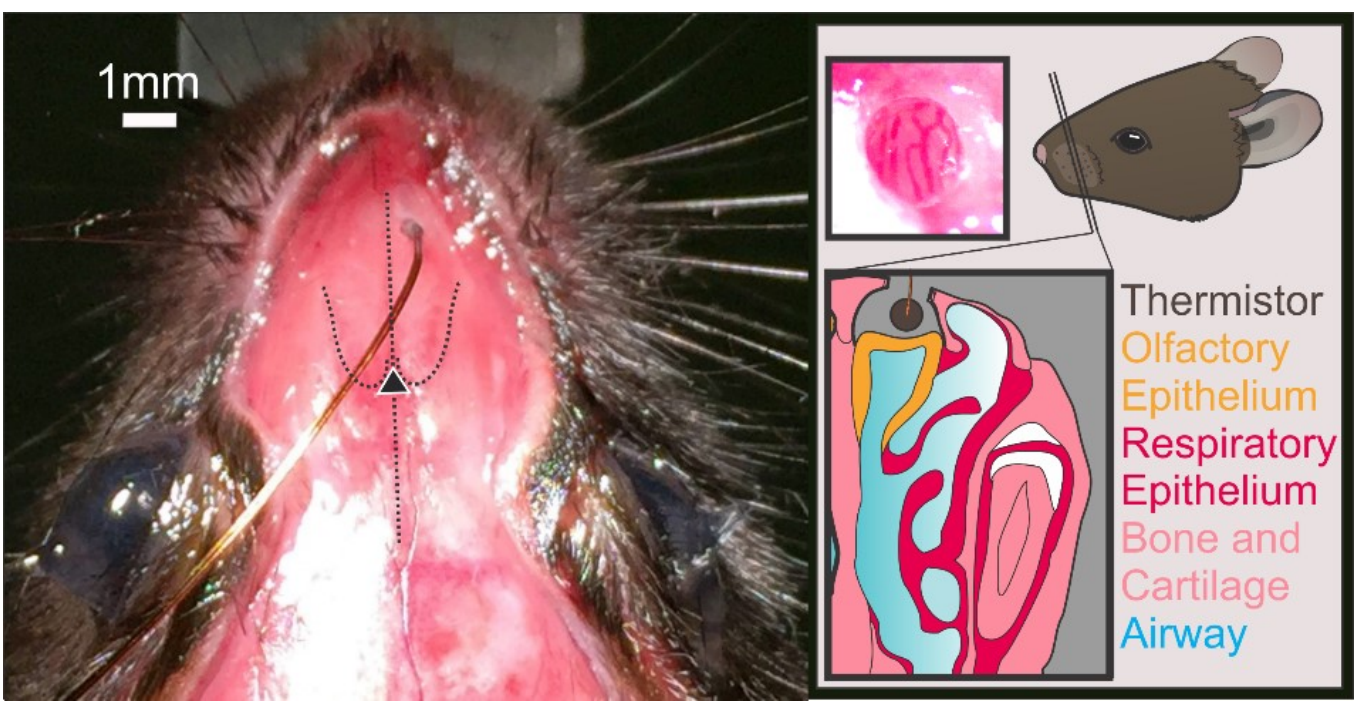

Figure 5-2. Surgical placement of thermistor probe.

Left panel: Picture of thermistor insertion. Dotted lines were added to highlight the locations of skull sutures used as landmarks for probe placement. Triangle indicates the point from which stereotaxic coordinates were measured; the intersection of the sagittal suture and the posterior edge of the nasal bone. Right panel: (Top left) High contrast image of soft tissues underlying the nasal bone. Poor attachment to the bone creates a space in which a thermistor can be easily implanted. (Right) Anatomy of a coronal section at this location. 

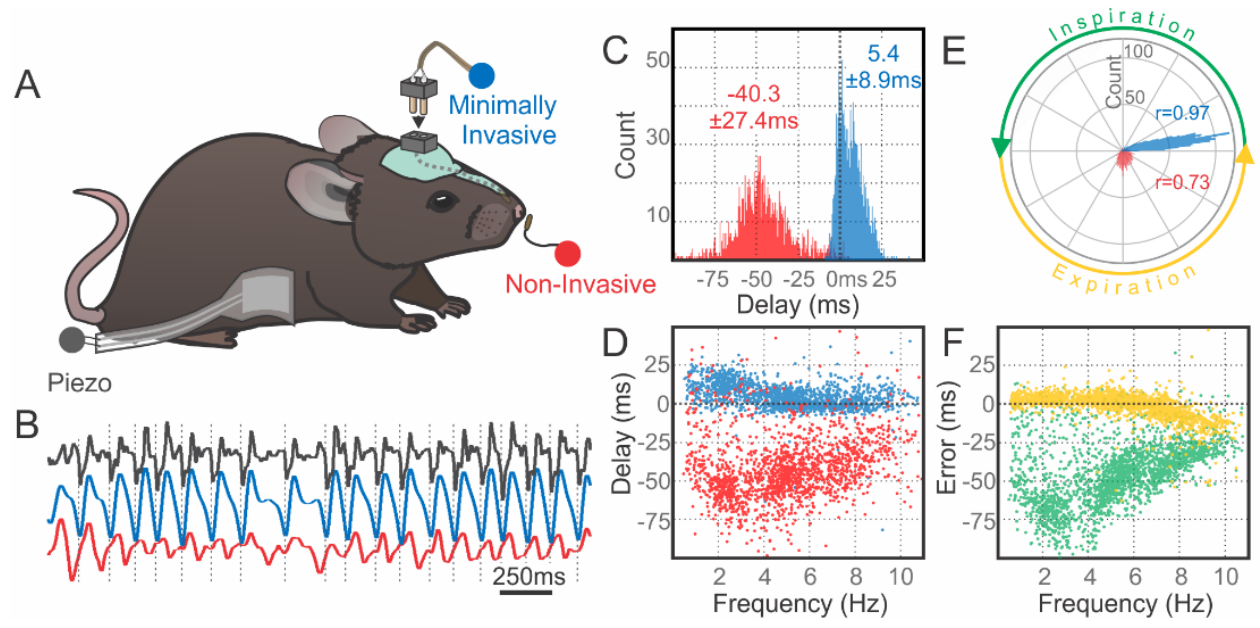

Figure 5-3. Comparison of respiration monitoring with minimally invasive and non-invasive thermistors in head-fixed mice.

A: Illustration of data sources for comparison (see text for details). B: Example of raw data traces (colors correspond to sensor colors in A) during both fast and slow breathing. Dotted lines indicate times denoted as the beginning of inspiration based on the piezo signal deflection. In the thermistor signals the beginning of inspiration corresponds to the troughs. C: Peri-event time histogram showing the detection time of inspiration in thermistors relative to the piezo. Extranasal thermistors had troughs that often preceded inspiration due to the constant cooling of the probe by room-temperature air, which counteracts the warming due to expiration. D: Scatter plot of detection delay of thermistor signals as a function of respiration frequency based on piezo measurements. Minimally invasive thermistors show only slight frequency-dependent delay during slow respiration. E: Comparison of phase estimates represented as circular data. Circular histograms consist of 360 1-degree bins. Index of straightness (Rayleigh's r) ranges from 0 to 1 , for evenly spaced or perfectly aligned data, respectively. These results indicate that the minimally invasive thermistor signal reliably represents the phase of respiration. F: Respiration frequency scatter plot of relative error of detection time for inspiration (green) and expiration (yellow) onset using extranasal and minimally invasive thermistors. Distributions indicate high degree of precision for external thermistors up to $6 \mathrm{~Hz}$ respiration (standard deviation $\pm 4.2 \mathrm{~ms}$ ) with error increasing to $\sim 25 \mathrm{~ms}$ for fast sniffing frequencies. 

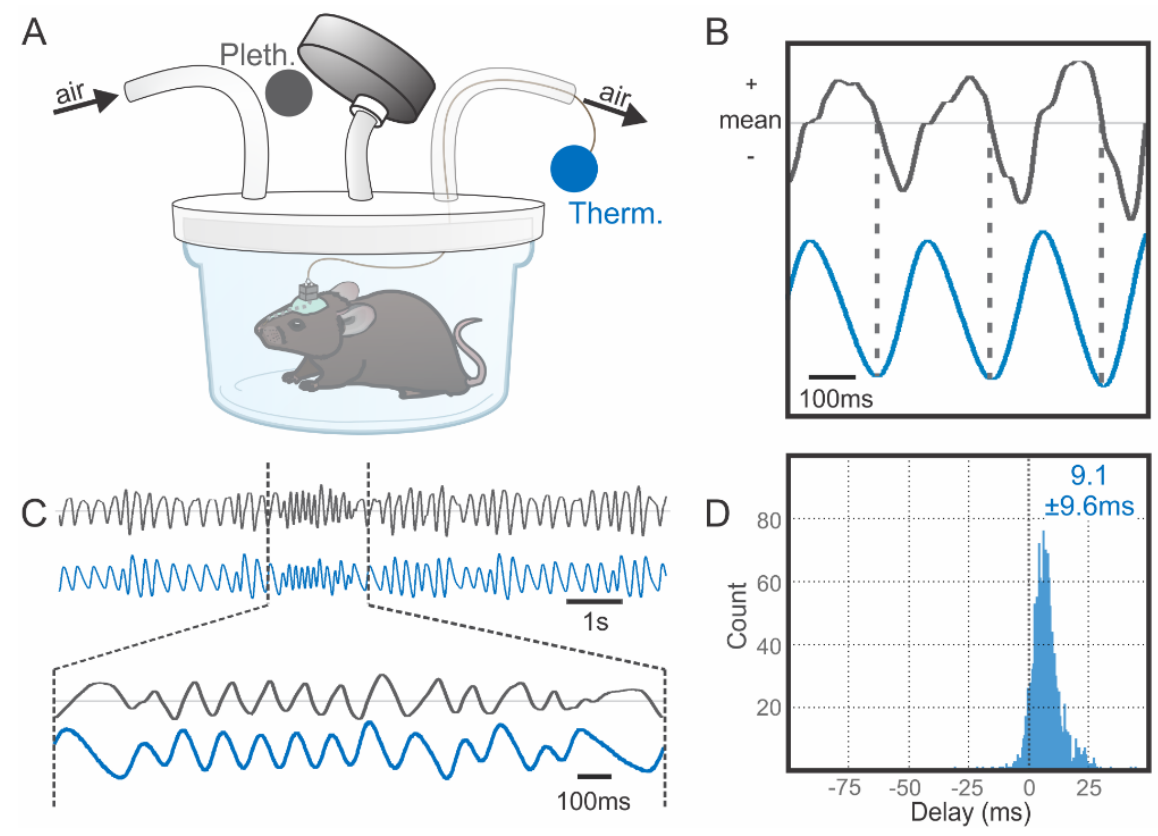

Figure 5-4. Plethysmographic recording of respiration during free movement. A: Illustration of recording setup. Mice were placed into a small plethysmography chamber while connected to the amplifier for thermistor measurements. B: Diagram for waveform interpretation. Initiation of inspiration appears as troughs in thermistor waveform, which correspond to transitions between above- and below-average pressure values in the plethysmography chamber. C: Example raw data traces of thermistor (blue) and plethysmograph (grey) signals. Thermistor measurements reliably matched plethythmograph measurements across a wide frequency range. Lower traces show the period of spontaneous sniffing marked in the top traces at an expanded timescale. D: Peri-event time histogram showing the detection time of inspiration in the thermistor relative to the plethysmograph. Results are comparable to those in the head-fixed condition. 
changes in chamber pressure. Voltage output from the demodulator was digitized at 2 kHz (CED 1401, Cambridge Electronic Design, UK) and stored on hard disk. Implanted probes were again connected to the amplifier via a thin $1 \mathrm{~m}$ long wire. Mice were then allowed to move freely for several minutes while thermistor and plethysmograph data were continuously recorded.

Recording of Sniffing Behavior. Effectiveness for measurement of spontaneous sniffing behavior during odor discrimination was assessed in a novel variant of an odor response task previously described in the literature. The odorants selected for exposure were ethyl tiglate (ET), ethyl butyrate (E4), acetophenone (Ac), citronellyl isobutyrate (Cit), ethyl valerate (E5), benzaldehyde (Bz), ethyl hexanoate (E6), and 2-heptanone $(2 \mathrm{H})$. Mice were habituated to head fixation on a treadmill in front of an odor port with continuous airflow before exposure to odorants. Once habituated, mice were exposed to the same odorant multiple times for 1 second durations at 1 minute intervals until they no longer responded with fast sniffing. The familiar odor was then alternated with a battery of novel odorants with a range of structural similarities, and respiration responses were simultaneously recorded.

Data Analysis. Data were analyzed in Spike2 (Cambridge Electronic Design, UK) or exported to Matlab (The Mathworks, U.S.A). All recordings were preprocessed in Spike2 by applying a smoothing filter with a time constant of $5 \mathrm{~ms}$; this was realized as a 20th order FIR filter with all coefficients equal to $1 / 20$. A DC removal filter was also implemented for both thermistor signals in order to subtract slow drifts in the temperature signal unrelated to respiration phase (see Figure 5-5C for example). DC removal was achieved by subtracting the result of the smoothing filter with coefficient of $50 \mathrm{~ms} \mathrm{(a}$ 200th order FIR filter with all coefficients equal to 1/200) from the original signal. Timing of respiratory events in the thermistor signals was compared to corresponding timing of events in the piezoelectric signal. Circular estimates of respiratory phase were created by normalizing each respiratory cycle duration to $2 \pi$ using the beginning of inspiration in the piezoelectric signal. Relative error of thermistor events was calculated using extranasal thermistor event time minus the corresponding event time in the minimally invasive thermistor. Mean chamber pressure was subtracted from plethysmograph signals using the same DC removal algorithm with a time constant of $500 \mathrm{~ms}$ so that 0 values correspond to mean chamber pressure. Frequency-dependent effects of respiration on waveform amplitude were not analyzed, as temperature changes can occur independent of airflow. Furthermore, the drift removal process reduces the amplitude of slower cycles, thus obscuring this relationship in the already tenuous link between nasal temperature and respiratory tidal volume. 


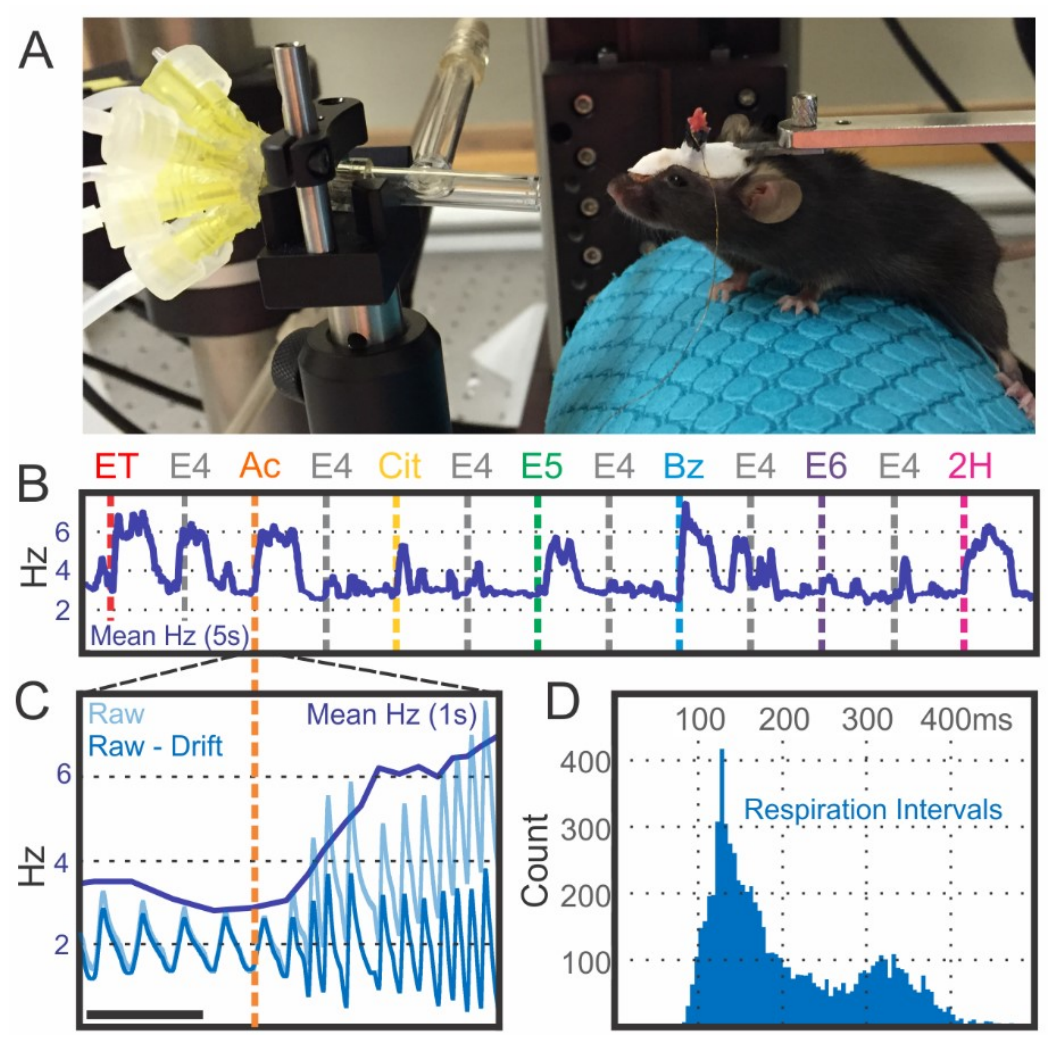

Figure 5-5. Measurement of sniffing behavior during odor presentation.

A: Photograph of the experimental apparatus showing the mouse on a treadmill with the glass odor port in front of the nares. B: Mean respiration frequency within a 5 second windows over the course of an experiment. Novel odors elicit sustained responses of high-frequency sniffing. Odorants were presented 1 minute apart for 1 second each. Dotted lines are color matched to the odorants listed in corresponding colors on top of the plot. Odor abbreviations listed in methods. C: Expanded time view of sniff rate with raw data for the second novel odorant, Acetophenone. Example 100ms DC removal was performed to aid in visualizing the sniff signal, since sniffing causes an overall cooling of the probe as air is rapidly exchanged through the nose. Black bar corresponds to 1s. D: Histogram of all respiration intervals during the recording session. The distribution is bimodal, corresponding to resting and sniffing respiration modes. 


\section{Results}

Comparison of Head-Fixed Recording Methods. A direct comparison of methods was made by recording respiration in head-fixed mice $(n=3)$ using three different methods simultaneously. The recording of chest expansion via a piezoelectric strip served as the reference as it provided the most direct and precise measurement of inspiration onset. Only periods of stable piezo measurements of chest movements were used for further analysis. The second signal came from a thermistor placed in front of the nares and the third from the minimally invasive implanted thermistor. The onset of downward deflections in the piezo signal was used to identify the beginning of chest expansion which corresponds to the beginning of inspiration. A direct comparison of the piezo and extranasal thermistor traces shows that the extranasal thermistor was less precise at determining the beginning of inspiration due to the constant cooling of the probe by room-temperature air. This led to consistently early estimates for the beginning of inspiration (Figure 5-3; delay of $-40.3 \pm 27.4 \mathrm{~ms}$ ). By comparison, respiration related temperature changes captured by the implanted thermistor more accurately reflected the correct moment of inspiration, with a delay of $5.4 \pm 8.9 \mathrm{~ms}$ (Figure 5-2C). Frequencydependent latency changes were subtle, and most apparent at low respiration frequencies (Figure 5-3D). Perhaps most importantly however, the response latency was not significantly altered by sniffing behavior, thus making the implanted thermistor appropriate for measurement of sniffing in a head-fixed preparation.

A direct comparison between the two thermistors showed highly synchronous detection of expiration onset, indicating a high degree of precision shared by both methods. This was especially apparent during resting breathing ( 0.5 to $6 \mathrm{~Hz}$; standard deviation $4.6 \mathrm{~ms}$ ), which suggests that extranasal thermistors can be highly effective for acquiring respiration data non-invasively, if expiration is used as the reference point for analysis.

Comparison of Freely-Moving Recording Methods. Respiration signals from the minimally invasive implanted thermistor were readily recorded in freely moving mice and without movement related artifacts in 3 of the 3 tested mice (see e.g. Figure 5-4). Direct comparison with simultaneously recorded plethysmography signals showed that the embedded thermistor signal reliably and accurately reflected respiration at all frequencies in the freely moving mice.

Assessment of Sniffing Behavior. Sniffing behavior was effectively measured during odor presentation in 3 head-fixed mice. The results obtained were very similar to those of Wesson et al., in several ways (Figure 5-5). Mice responded to novel odors with sustained periods of high-frequency sniffing, whereas they responded to familiar odors with only brief, less pronounced increases in respiration frequency. Additionally, odorants that were structurally similar to a repeated odorant elicited smaller increases in sniff frequency compared to structurally dissimilar odorants (Figure 5-5B, E5 and E6 
versus Bz). Lastly, mice also demonstrated characteristic dishabituation to a familiar odor following the presentation of the first novel odor (Figure 5-5B, sniffing after first E4).

Together, these results show that the minimally invasive thermistor implant is as effective a tool for the assessment of sniffing in response to odor as the pressure transducer connected to an intranasal sniff cannula employed by Wesson et al. ${ }^{209}$

\section{Discussion of Method}

Current approaches to measuring mouse respiratory movements rely either on measuring temperature changes associated with inhaling cool air and exhaling air warmed up during passage through the airways and lungs, or they use miniature pressure transducers to measure respiration related pressure changes in the nasal cavity via implanted cannulae. Placing a thermal probe directly in front of the nares is the least invasive method, but is only precise when used to detect the onset of expiration during resting breathing $(<6 \mathrm{~Hz})$. Implanting thermistors into the nasal cavity gives reliable measurements at all frequencies but interferes with the airflow and irritates the sensitive lining of the nasal cavity. Intranasal pressure measurements have the same drawbacks as they too require invasion of the nasal cavity with a small metal or plastic cannula and, in addition, add considerable weight to the nose and partially obstruct the visual field.

Another noteworthy approach employs a pressure sensor implanted into the thoracic cavity to detect changes in pressure associated with respiratory movements. This method is advantageous in that it does not cause damage to olfactory tissues. However, the temporal precision with which inhalation onset times were detected declined considerably with decreasing respiratory frequency while precision of detection was stable across all frequencies for the minimally invasive thermistor (Figure 5-3D). In summary, this novel method of respiration monitoring in mice using minimally invasive thermistor implants is at least as reliable as current methods using implanted sensors, but without the drawbacks, such as obstruction of airflow or damage to the nasal epithelium. Minimally invasive thermistor implants thus allow highly precise respiratory monitoring with reduced pain and stress for the animals and without interfering with natural breathing behavior.

\section{Respiratory Modulation of LFPs and Spikes in the Mouse Visual System and Ancillary Regions}

With respiration effectively measured, simultaneous recording of respiration with LFPs and spikes can be used to detect links between neuronal activity and breathing. These links will be statistically evaluated in various brain regions in order to characterize respiratory modulation of neuronal activity in specific areas of the mouse brain. Furthermore, the contribution of the OB to these respiration-locked oscillations can be assessed by surgical removal of the $\mathrm{OB}$, or by disrupting activity within it. In this case, the less invasive route was taken by administering Clozapine-N-Oxide (CNO) to mice with inhibitory Designer Receptors Exclusively Activated by Designer Drugs 
(DREADDs) ${ }^{210}$ expressed in the OB. When this drug is given systemically, inhibitory DREADDs channels in CamKIIa-positive neurons of the granule cell layer of the bulb become activated $^{211}$, leading to a disruption of the OB respiratory rhythm (Figure 5-6). Thus, when the drug is found to effectively abolish respiration-locked rhythms in other regions of the brain, it becomes evident that they are dependent upon the intact OB.

Given that a previous emphasis was given on how neuronal oscillations in LGN and V1 support neuronal communication related to visual processing, I will focus this section on the apparent effect of respiration on oscillations in those areas, as well as on areas that are known to influence oscillations in LGN or V1. This includes the somatosensory area for the trunk, which is adjacent to V1 and may interact with it via cortico-cortical or cortico-thalamocortical connections ${ }^{212}$, as well as the basal forebrain, which provides cholinergic input to $\mathrm{V} 1$ and disinhibits $\mathrm{LGN}^{213,214}$. It is necessary to understand the extent to which respiration affects neuronal activity in these brain areas so that we can understand the role of respiration-locked sensory input in the brain, and how it may affect functions like visual processing. I will begin by describing some key findings that suggest that respiration drives neuronal activity patterns within these regions, and then follow with the effect of OB output on the synchronous gamma oscillations that occur during visual processing.

The most conspicuous way in which breathing affects brain activity is by driving oscillations that occur at the respiratory frequency. In mice, this is typically in the $2-4 \mathrm{~Hz}$ frequency range at rest, or $8-12 \mathrm{~Hz}$ range during sniffing. The presence of respirationlocked oscillations is easily discernible based on cross-correlation analyses of the two waveforms, or by LFP averages at the peaks or troughs of the respiration waveform, for respiratory cycles of a similar time interval. Spike rates relative to respiratory peaks or troughs are also averaged to identify relationships between respiration and neuronal output within a region. Of equal importance is the modulation of gamma oscillation amplitude in phase with the respiratory rhythm. These patterns have been identified in several brain regions, as follows.

\section{Neocortical Regions}

As laid out in Ito et al. $(2014)^{90}$, non-olfactory regions of the neocortex exhibit prominent respiration-locked oscillations. Since this publication and before my time within them, the Heck lab identified respiration-locked slow oscillations and spike rate modulations in the whisker motor cortex, the prefrontal cortex, and the primary visual cortex of the mouse (Figure 5-1).

The modulation of visual cortical activity by respiration was of particular interest, as visual perception is not typically thought of as occurring rhythmically, especially at a slow rhythm. However, after investigation in many animals, the modulation of visual cortical activity at rest appeared to be inconsistent and insubstantial (Figure 5-7).

Statistically significant relationships between respiration and the LFPs could be identified with highly sensitive directional statistics (Figure 5-7G), but in the absence of strong 
a
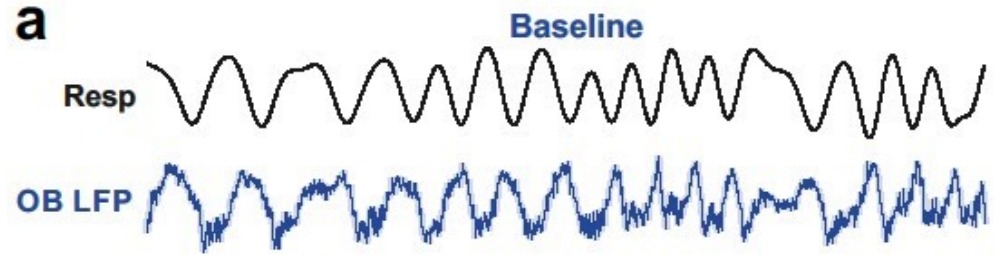

b ILFP: 100NV

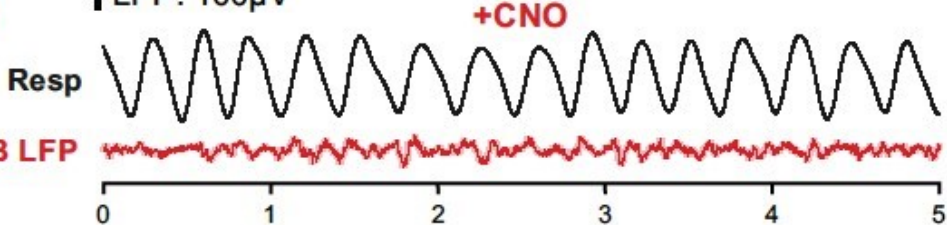

C

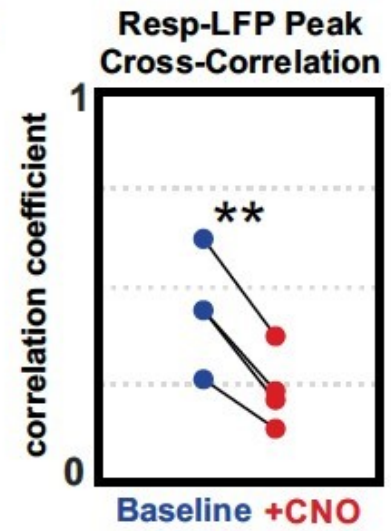

Figure 5-6. Disruption of olfactory bulb respiration oscillations with administration of inhibitory DREADDs channel activator Clozapine-N-oxide (CNO).

(a) Baseline example of simultaneously recorded olfactory bulb LFP and respiration shows robust neuronal oscillations in OB locked to the respiratory cycle. Time and voltage scale equivalent to b. (b) Example of olfactory bulb LFP after systemic injection of CNO solution. Respiration-locked neuronal oscillations are visibly disrupted. Time is shown in seconds. (c) Group data showing disruption of respiration-locked OB activity following $\mathrm{CNO}$ injection. Peak correlation values were taken to account for variable latency of respiration-driven oscillations. 10 minutes of resting data were used to calculate each cross correlation. Paired T-test:** $\mathrm{p}<0.01$. 
A

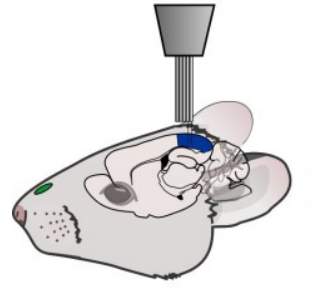

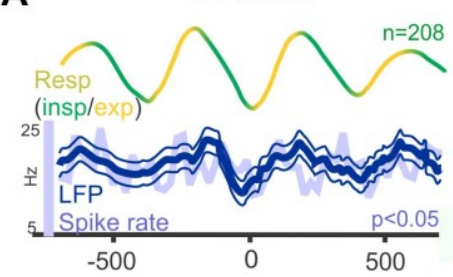

OB Saline

B OB Lidocaine

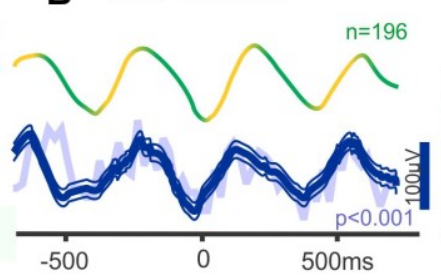

OB hM4Di Baseline
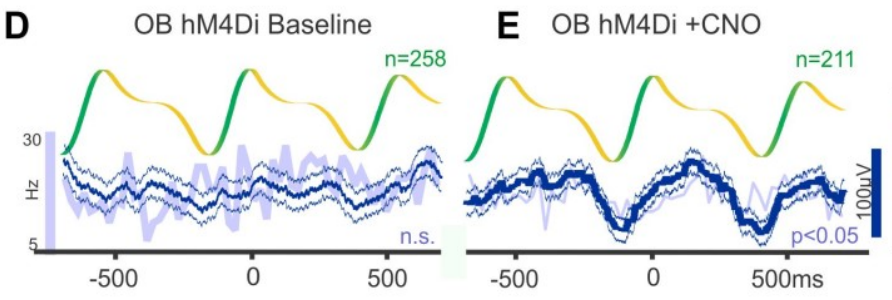

$\mathbf{F}$

OB inhibition example

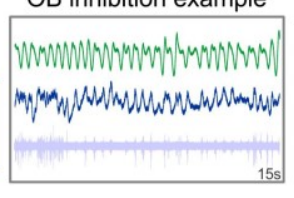

OB inhibition example

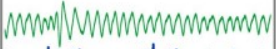

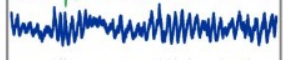

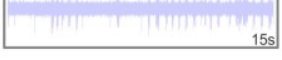

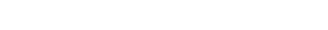
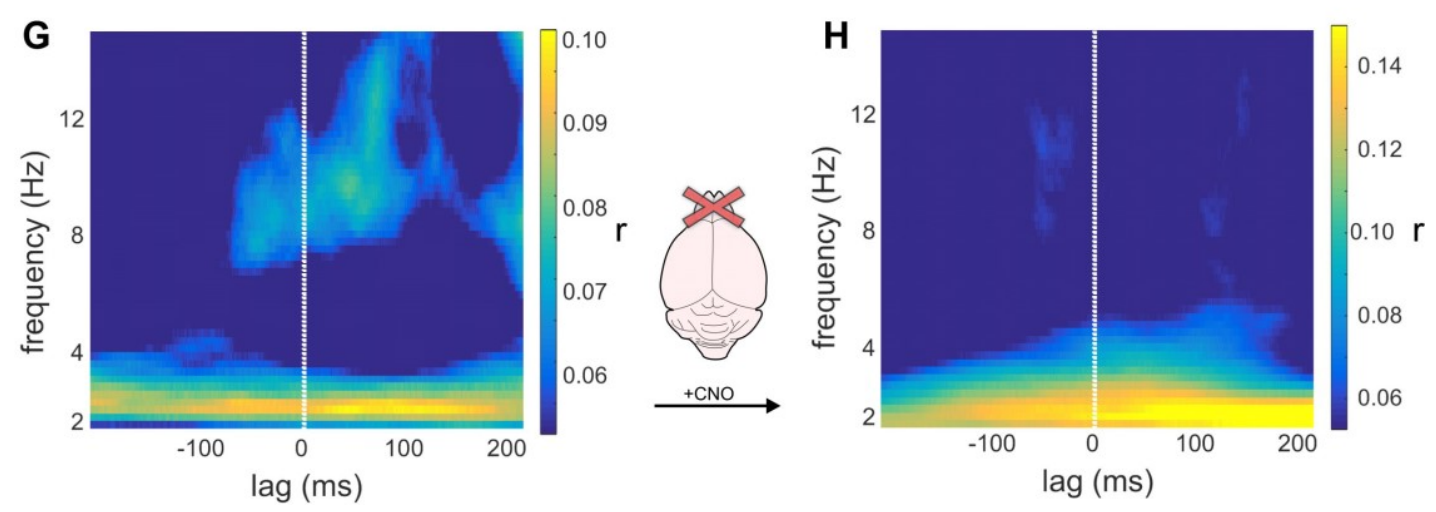

Figure 5-7. Respiration-locked slow oscillations in the visual cortex and their dependence on $\mathrm{OB}$ activity.

Recording from layer 4 of V1 with simultaneous recording of respiration reveals complex relationship to OB activity. (A,D) Dark traces show LFP averages aligned with the end of inspiration with surrounding lines for standard error. Light purple trace shows spike rate averaged. Probability for spike-respiration correspondence was calculated using a Rayleigh test. Baseline measurements reveal only a slight correlation between respiration and neuronal activity. (B,E) Similarities of LFP and spikes with the respiratory cycle after OB inhibition. The olfactory bulb was inhibited using both lidocaine application (B) and systemic $\mathrm{CNO}(\mathrm{E})$. Correlations between respiration and V1 activity increased relative to baseline following olfactory bulb inhibition by both methods. (C,F) 15 second raw data examples of respiration, V1 LFP, and V1 spikes following OB inhibiton by lidocaine (C) and CNO (F). Correlations between LFP and spikes as well as LFP and respiration are visible even by crude inspection in these brief windows. (G) Frequencyresolved two-sample $r$ test of V1 LFP aligned with the end of inspiration, with threshold of $p<0.001$. Strong correlation is detected at the respiratory frequency in this sensitive statistical test, as amplitude is not factored into the analysis. Interestingly, a significant component is also detected above the respiration frequency between 8 and $12 \mathrm{~Hz} .(\mathrm{H})$ Same test after CNO for OB inhibition. Correlation becomes more consistent following $\mathrm{OB}$ inhibiton, illustrated by the greater $\mathrm{r}$ value, and higher frequency relationship disappears. 
spike rate modulation or high amplitude LFP averages it is unlikely that phasic input from respiration in the normal mouse brain has a strong influence on visual processing. Curiously, stronger modulation of V1 LFPs and spikes in phase with respiration following OB inhibition by both lidocaine (Figure 5-7B and C) and CNO (Figure 5-7E and $\mathbf{F}$ ) was observed. This suggests a complex relationship between the visual cortex and respiration-locked neuronal activity, which will require much more thorough experimentation in the future to untangle. In the meantime, some early results related to the transmission of information from the lateral geniculate nucleus (LGN) to the visual cortex suggests $\mathrm{OB}$ output might play a role in maintaining cortical tone. This will be discussed in more detail later.

Immediately anterior to the visual cortex is a region that receives somatosensory input from the trunk of the mouse. With every breath this region reliably generates a slow oscillation and a burst of high frequency spike activity (Figure 5-8). These oscillations and spikes are aligned with moments of peak tidal volume, corresponding to excitatory input from pulmonary stretch receptors. Thus, they are likely distinct in origin from the olfactory bulb, but this requires further investigation.

\section{Lateral Geniculate Nucleus}

The visual relay nucleus of the thalamus exhibits strong respiration-locked oscillations and spikes, with the modulation of gamma frequency activity and spike rate appearing more prominent than the entrainment of respiratory-frequency oscillations. At rest, while the mouse gazes passively at a blank gray screen, bursts of high gamma $(>50 \mathrm{~Hz})$ occur with a significant phase preference within the respiratory cycle. Interestingly, when OB activity is disrupted, the degree of phase amplitude coupling becomes even greater (Figure 5-9). This suggests that there is a second distinct source of respiration-locked activity driving gamma oscillations in the thalamus. I hypothesize that the most likely sources of this activity are the brainstem and the somatosensory afferents from the trunk.

The trunk afferents may be particularly disruptive to thalamic gamma activity, as the somatosensory and visual areas of the thalamus receive common recurrent input from the thalamic reticular nucleus (TRN). The TRN is essentially a shell of inhibitory neurons surrounding the thalamus, all of which fire with precise synchrony ${ }^{16}$ due to extensive interconnection via gap junctions ${ }^{215,216}$. As a consequence, gamma oscillations are practically uniform in strength and timing throughout the thalamus. These neurons are collaterally excited both by thalamocortical and corticothalamic projections when sensory impulses arrive. Together, this leads to the conclusion that increased PAC may be caused by an imbalance between two sources of respiration-locked input to the thalamus, with somatosensory sources dominating after the $\mathrm{OB}$ is disrupted, entraining the thalamic gamma rhythm to only a single phase of the respiratory cycle. In the somatosensory whisker cortex, gamma activity reaches its peak amplitude near the end of expiration, which would offset trunk afferents in terms of phase. 

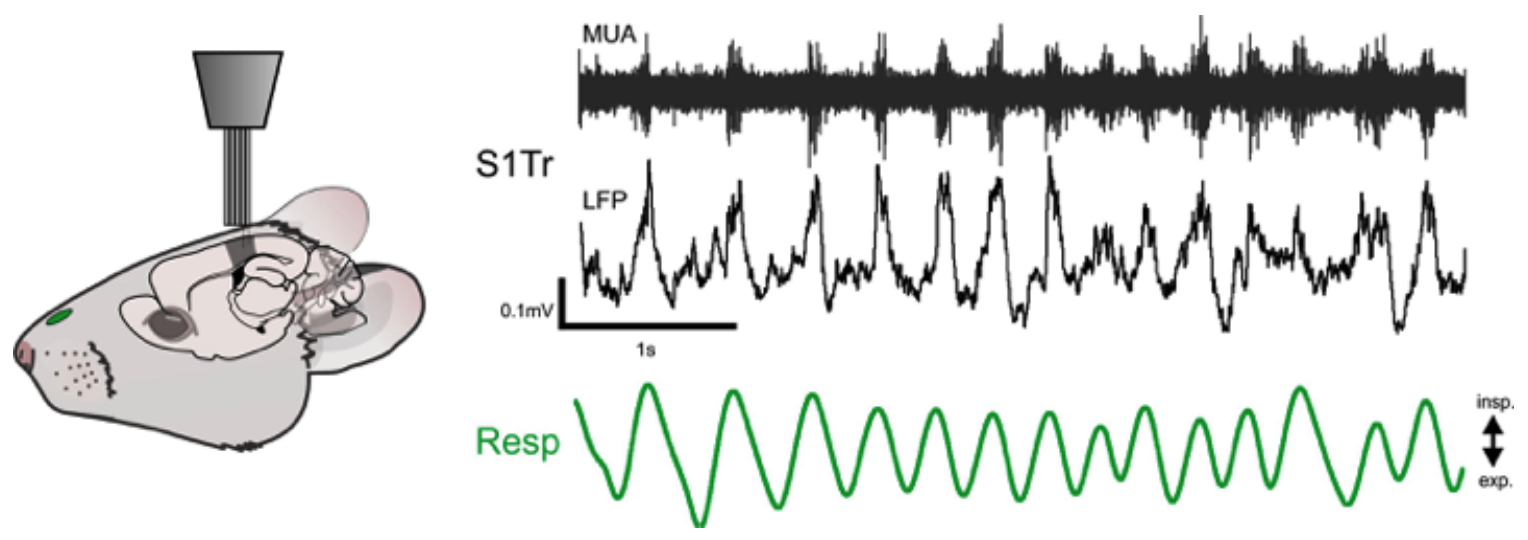

Figure 5-8. Respiration-locked slow oscillations and spike activity in the primary sensory cortex for the trunk/abdomen (S1Tr).

Traces show five second example recording of LFP and multi-unit activity (MUA) with simultaneous respiration (Resp). Local activity in this region corresponds very closely to the respiratory rhythm, with the highest rate of spike activity occurring at the end of inspiration. This likely corresponds to excitatory input from pulmonary stretch receptors when the lungs are filled with air. 

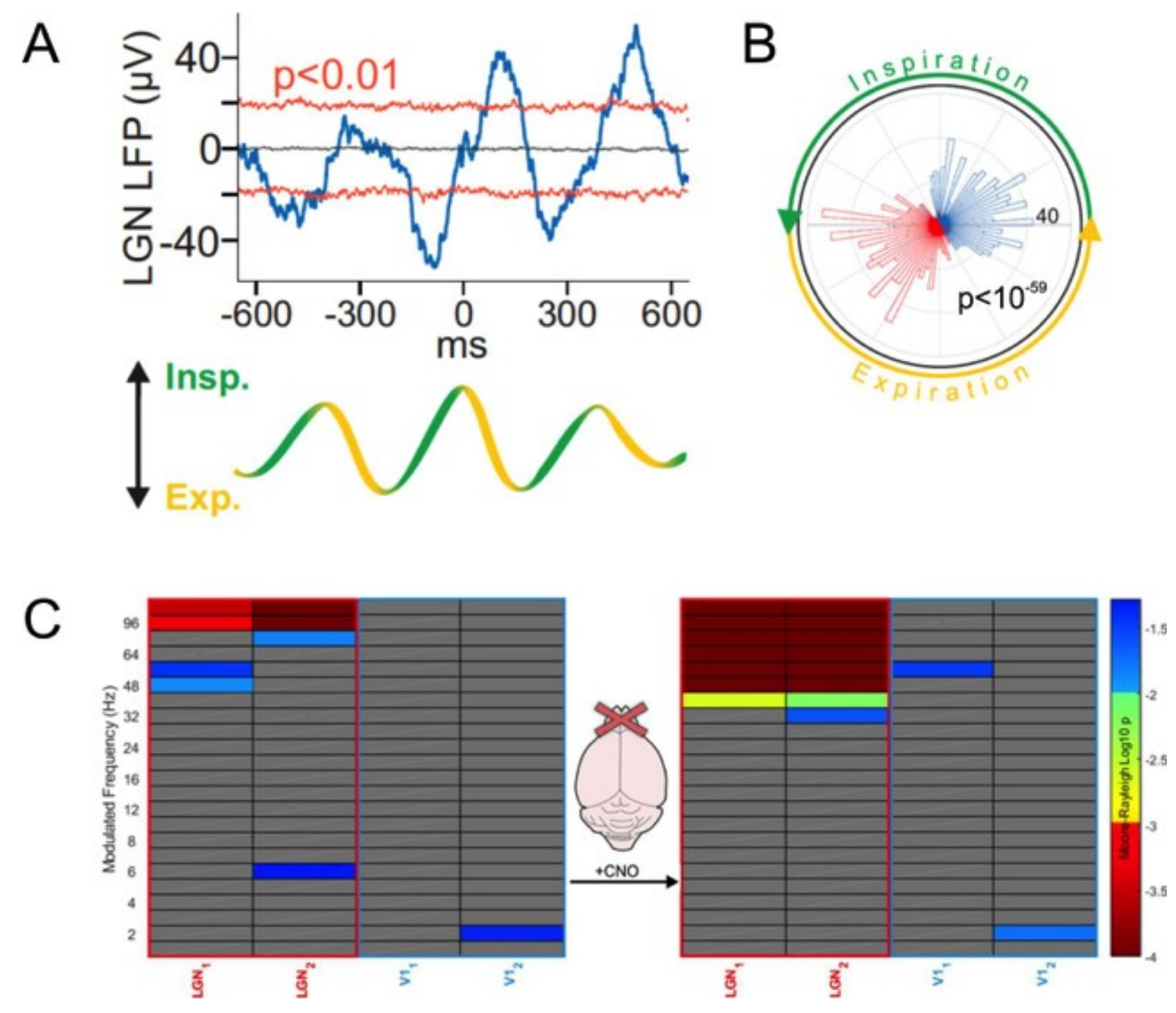

Figure 5-9. Respiration-locked activity in the LGN.

(A) Blue trace shows average of LGN activity aligned on the end of inspiration with red $99 \%$ confidence intervals determined by bootstrap analysis. At baseline there is a significant correlation between slow oscillations in the LGN and respiration. Respiration average with error shows in yellow and green below. (B) Modulation of spike activity at rest is highly significant, as determined by a 2-sample binned $r$ test. Blue angular bins correspond to a greater number of spikes than expected, and red corresponds to fewer than expected. Spikes appear to disproportionately occur at the beginning of inspiration. (C) Respiration PAC analysis of LGN and V1 LFP using Moore's modified Rayleigh approach. Gray boxes are not significant, blue are $p<0.05$, green $p<0.01$, red $p<0.001$, and maroon $p<0.0001$. At baseline, oscillations in the high gamma range $(>90 \mathrm{~Hz})$ are strongly modulated in phase with respiration. Modulation becomes greater after OB inhibition by $\mathrm{CNO}$, also spanning a broader frequency range $(36-100 \mathrm{~Hz})$. 
The brainstem also oscillates with respiration as it contains the pre-Bötzinger complex, the pattern-generating nucleus responsible for the execution of breathing behavior $^{217}$. Respiration-locked activity can be observed throughout the brainstem due to interconnected nuclei, yet these oscillations are ablated when the pre-Bötzinger complex is removed or silenced ${ }^{217}$. Some of these medullary nuclei have been shown to project to the thalamus ${ }^{218}$, including the LGN and TRN ${ }^{219}$. These reafferent projections could be responsible for augmented thalamic PAC after OB inhibition in one or both of the following ways: These impulses could excite thalamic nuclei and directly entrain a gamma rhythm, or these impulses could excite the TRN, which indirectly entrains the gamma rhythm. Further experiments are needed to fully understand the relationships between these sources of respiration-locked input and the aggregate effects on their targets.

\section{Basal Forebrain}

The basal forebrain (BF) contains many neuronal subtypes with diverse neurotransmitters and projections, all of which exhibit oscillatory activity that is highly correlated with respiration $^{187}$. In order to better understand the source of these respiration-locked oscillations, a series of recordings were performed in mice with inhibitory DREADDs in the OB. Baseline measurements of BF LFPs were analyzed and compared with post-CNO recordings for their relationship to respiration. At baseline, BF LFP was highly correlated with the respiratory measurement, and LFP averages aligned with the peak of the respiration signal revealed a strong oscillatory component (Figure 5-10). After baseline measurement, $\mathrm{CNO}$ was administered to the mice, disrupting the respiration OB rhythm. After the drug had taken effect, a second recording was made, revealing a diminished relationship between respiration and BF activity (Figure 5-10C and $\mathbf{E}$ ). This strongly suggests that respiration-locked oscillations in the BF are driven by output from the olfactory bulb, either directly or via projections from the piriform cortex.

The projections from the BF are thought to play an essential supporting role in sensory processing and cognition. Cholinergic neurons in the BF provide acetylcholine (ACh) to the entire neocortical mantle, and optical stimulation of these terminals alone can lead to the emergence of gamma oscillations. Furthermore, ACh-induced gamma has gained prominence in visual processing research recently, as BF activation was shown to drive gamma oscillations in the visual $\operatorname{cortex}^{220}$ and increases the precision and consistency of evoked cortical responses ${ }^{221}$. Interestingly, cholinergic neurons in the BF are activated by neurons in the brainstem concurrently involved in the initiation of locomotion $^{220,222}$, and additionally enhance visual evoked responses ${ }^{222}$. If ablation of BF respiration oscillations reduces the output from ACh neurons to the cortex, it is likely that this will affect both visual-evoked cortical activity and visual processing. While a direct link between the two is not currently conclusive, evidence of disruption of cortical activity and visual perception by olfactory bulb inhibition has been identified. 

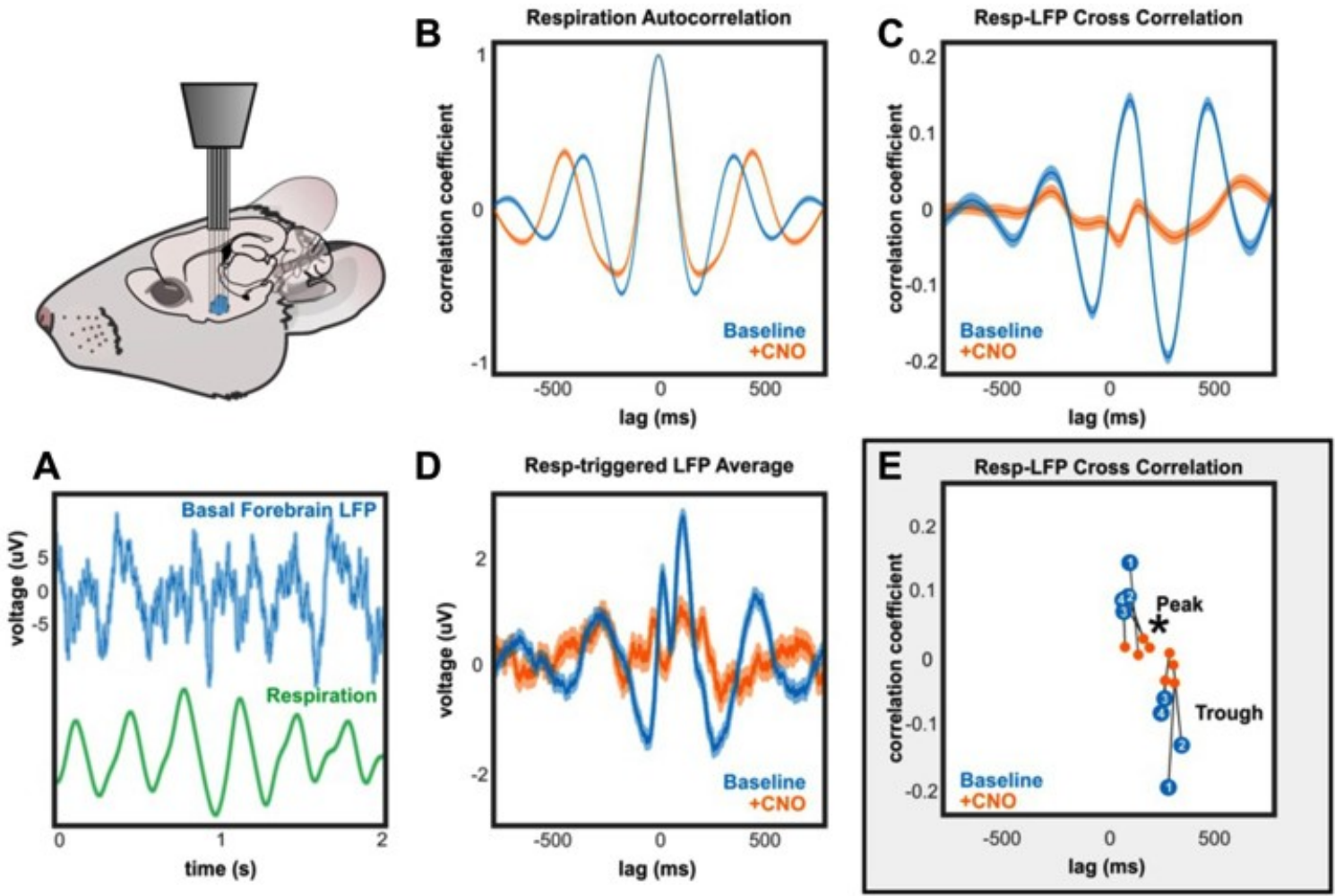

Figure 5-10. Respiration-locked slow oscillations in the basal forebrain (BF) before and after olfactory bulb inhibition by CNO.

(A) Raw data example of BF LFP and its correspondence to respiration. (B) Autocorrelation of respiration waveforms showing slight slowing of respiratory rhythm after $\mathrm{CNO}$, but otherwise little change. (C) Example cross correlation between respiration and BF LFP before and after CNO. Correlation is high at baseline and diminished after CNO. (D) Example LFP average aligned on the end of inspiration. Amplitude of LFP average is diminished after CNO administration. (E) Group data showing change in Respiration-LFP cross correlations after CNO administration. Dots correspond to the correlation values and time lags of the peaks and troughs in the correlations. Peak-totrough differences significantly change after OB inhibition, suggesting BF respiration oscillations are driven by the OB. 


\section{Disruption of Thalamocortical Phase Synchronization and Visual Acuity by OB Inhibition}

In Chapter 3, evidence was presented to support the hypothesis that oscillatory phase synchronization supported the effective transmission of information from LGN to V1. This predominantly occurred in the high gamma $(50-90 \mathrm{~Hz})$ frequency range during the processing of a black and white checkerboard image.

In order to determine whether the normal electrophysiological patterns associated with visual processing depend upon respiration-locked oscillations in $\mathrm{OB}$ and subsequent excitation of downstream targets, I performed simultaneous recordings of LGN and layer 4 of $\mathrm{V} 1$ before and after $\mathrm{CNO}$ administration in mice with inhibitory DREADDs expressed in OB neurons. A second group of wild-type mice also underwent the recording procedure, during which they were injected with vehicle in order to control for the effect of stimulus habituation and stress from injection on stimulus processing. As it was described in detail in Chapter 2, a novel phase synchronization analysis was developed and used to quantify the effect of high contrast visual stimulation on LGN-V1 gamma phase synchronization. Peak $r$ values occurring within the $50-90 \mathrm{~Hz}$ frequency range and the 50-150 ms latency range were taken for each condition, and post-injection values were compared to baseline values.

\section{Results}

Mice expressing the inhibitory DREADDs channel in OB neurons exhibited a consistent decrease in phase synchronization following the injection, whereas wild-type mice did not, as determined by a paired t-test within each group (Figure 5-11A and $\mathbf{B}$, $\mathrm{p}=0.041$ DREADDS, $\mathrm{p}=0.967 \mathrm{WT}$ ). In order to determine whether this treatment produced a corresponding change in visual processing and perception, visual acuity threshold was also measured in two sets of mice using machinery designed to measure the optomotor response in rodents ${ }^{223}$. The first set of mice expressed inhibitory DREADDs in neurons of the OB, while the second set did not. Both sets of mice received doses of CNO.

DREADDs-positive mice experienced a corresponding decrease in visual acuity threshold, whereas wild-type mice receiving $\mathrm{CNO}$ did not, as determined by a paired ttest within each group (Figure 5-11C and $\mathbf{D}, \mathrm{p}=0.010$ DREADDs, $\mathrm{p}=0.295$ WT).

\section{Discussion}

Together, the changes in thalamocortical synchronization and visual acuity suggests that normal visual perception depends upon respiration-locked output from the OB. Phase synchronization of high gamma oscillations in LGN and V1 was demonstrated previously to be a prominent feature of normal visual processing, and these oscillations were uniquely correlated with the occurrence of highly synchronous spikes in the two 
Figure 5-11. Effects of CNO and olfactory bulb inhibition on thalamocortical phase synchronization and visual perception.

(A) Examples of thalamocortical phase synchronization in response to visual stimulation before and after experimental treatment, as detected by the binned 2-sample $r$ test. Color plots have had a statistical threshold applied so that only $r$ values corresponding to $\mathrm{p}<0.001$ are revealed. Top two show no change in consistency of synchronization after injection with vehicle, whereas bottom shows disruption of phase synchronization after systemic CNO to inhibit olfactory bulb activity. (B) Comparison of changes in peak $r$ values after treatment with vehicle and CNO. No change was detected in synchronization after vehicle, suggesting no effect of stimulus habituation or injection stress. Consistent disruption of synchronization was identified after CNO. Phase synchronization of gamma oscillations was shown to correspond to synchronization of neuronal spike activity in the two regions, suggesting $\mathrm{OB}$ disruption may have a negative effect on visual processing. (C) Illustration of behavioral testing procedure to detect visual acuity. Mice are placed in a chamber surrounded by computer monitors, which display rotating bars of variable spatial frequency. Rotating bars elicit a cortex-dependent optomotor reflex when visible to the mouse, thus the experimenter can observe the reflex to infer perception of the stimulus in order to find the visual threshold. (D) Results of experimenter-blinded visual acuity testing in two groups receiving CNO, one with inhibitory DREADDs in the OB and a control group without. Mice with OB DREADDs experienced an acute deficit in visual acuity following $\mathrm{CNO}$ administration relative to baseline measurements with vehicle. Thus, the treatment that resulted in disruption of gamma phase synchronization caused a corresponding deficit in visual processing, suggesting that the respiration $\mathrm{OB}$ rhythm is necessary for normal visual performance. 


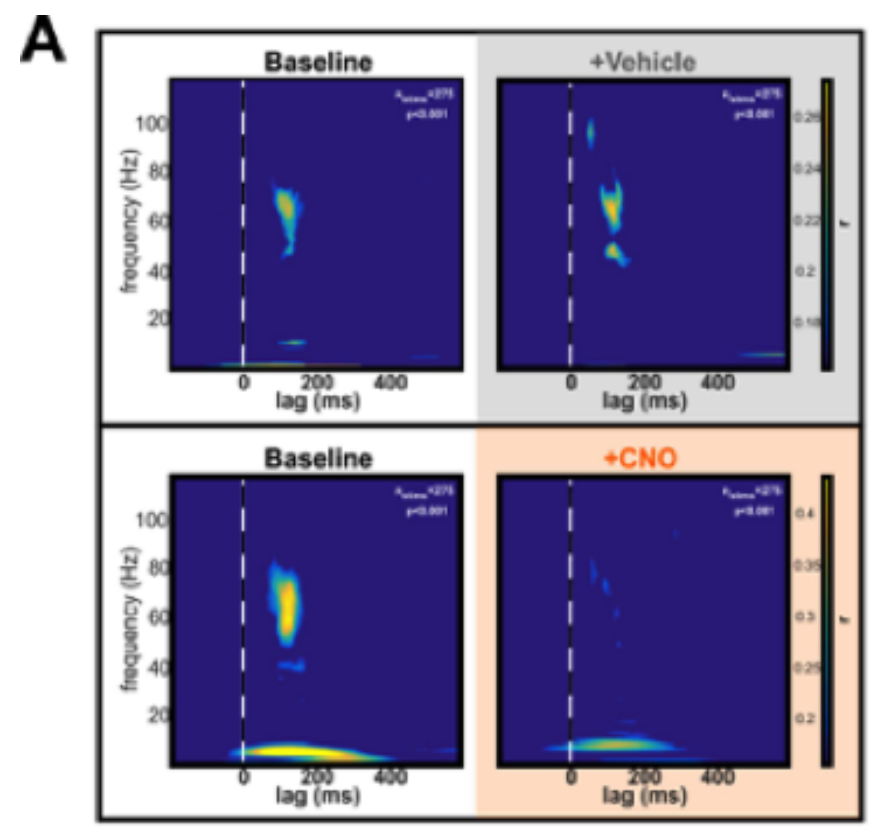

B

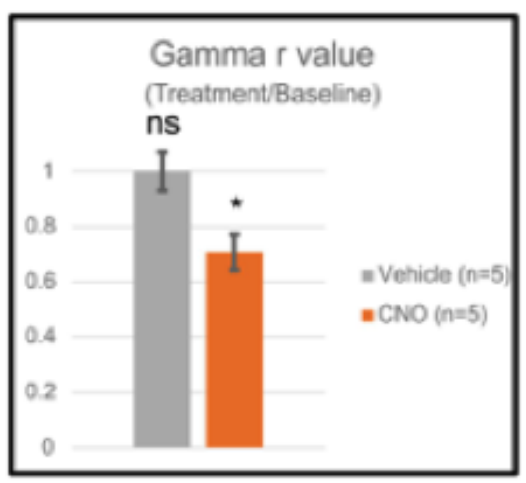

C

D

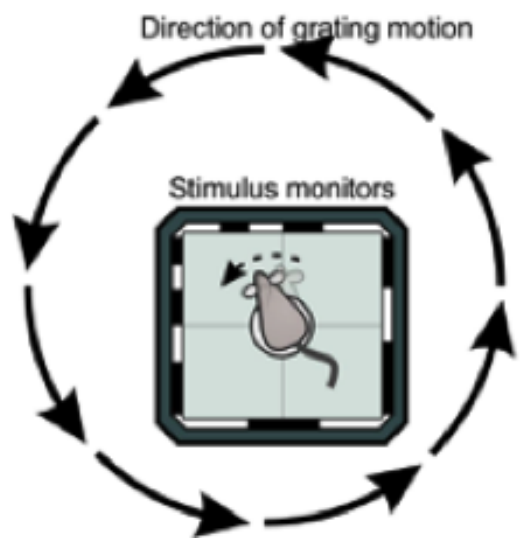


regions. Evidence that $\mathrm{OB}$ inhibition causes an acute decrease in visual acuity suggests that disruption of phase synchronization leads to a disruption in visual processing.

The anatomical link that accounts for the disruption in visual processing remains to be fully understood, but a few possibilities are suggested by our current understanding of respiration-locked oscillations throughout the brain. For example, it was demonstrated that the basal forebrain exhibits respiration-locked oscillations, and that these are driven by OB output. Cholinergic neurons within the BF have been shown to initiate high gamma oscillations in the visual cortex with approximately the same frequency profile as those related to thalamocortical transmission. Thus, a disturbance in ACh output may result in an inability of the cortex to properly resonate in a synchronized manner with LGN. Additionally, I found that OB inhibition lead to abnormal gamma frequency PAC in the thalamus at rest. During visual processing, this could possibly lead to an inability of LGN to provide stable oscillations with which V1 can synchronize. Many anatomical connections could be involved in mediating these effects, including reciprocal connections between brainstem and $\mathrm{BF}$ with distinct sources of respiration oscillations, brainstem projections to the TRN, BF projections to the TRN, somatosensory cortical projections to the TRN, BF projections to the cortex, and corticocortical and corticothalamocortical connections between adjacent somatosensory areas and visual cortical areas. Each of these projections will have to be selectively perturbed in order to understand its contribution.

The control condition in the electrophysiological experiments was the administration of vehicle rather than $\mathrm{CNO}$, however it was recently revealed that $\mathrm{CNO}$ is metabolized into clozapine, a fairly potent drug used to treat schizophrenia. Following this discovery it seems essential for further studies to repeat the experiments with CNO as a control. Therefore, the results herein should be interpreted with consideration for the possibility that CNO rather than DREADDs is responsible for the changes in thalamocortical synchrony that were observed.

\section{Respiratory Entrainment of Hippocampal Sharp-Wave Ripple Activity in CA1**}

The hippocampus, a structure essential for memory function, is not formally a part of the olfactory system, but it receives inputs from the piriform cortex via the entorhinal cortex and exhibits respiration-locked oscillations ${ }^{189,190,224}$. The hippocampal network generates characteristic sharp-wave ripple (SWR) activity which has been shown to be critically involved in memory consolidation and memory retrieval in mice ${ }^{225-227}$ rats $^{228-235}$ and non-human primates ${ }^{236-238}$.

\footnotetext{
**Modified with permission of Nature Publishing Group in the format Thesis/Dissertation via Copyright Clearance Center. Liu, Y., McAfee, S. S. \& Heck, D. H. Hippocampal sharp-wave ripples in awake mice are entrained by respiration. Sci Rep 7, 8950, doi:10.1038/s41598-01709511-8 (2017) ${ }^{239}$.
} 
Here it was asked whether hippocampal SWR activity is modulated by respiration and whether respiratory influence on SWR activity required OB activation. To this end extracellular recordings were performed in the dorsal hippocampal CA1 region in awake head-fixed mice while simultaneously monitoring respiration. The role of olfactory bulb activity was evaluated using designer receptors exclusively activated by designer drugs (DREADDs $)^{210}$ to inhibit respiration-locked activity in the OB.

\section{Results}

Recordings of hippocampal SWR activity were conducted in nine mice (Figure 5-12). Five mice were assigned to a control group and 4 mice to a group that received bilateral injections of a viral vector construct that caused the expression of DREADDs in afferent projection to the main $\mathrm{OB}$. This allowed us temporarily suppress of respirationlocked $\mathrm{OB}$ activity by systemic injection of $\mathrm{CNO}$, as verified in separate experiments involving recordings of OB activity in an additional 4 mice (Figure 5-6).

In the control group of 5 mice a total of 382 SWRs were detected during periods where the mouse was at rest. The waveform of the averaged raw SWR activity showed the characteristic combination of a negative deflection and high-frequency oscillations (Figure 5-13a). Time-Frequency analyses of LFPs around the SWRs indicated that the detected SWRs had same peak frequency and duration as reported in literature (Figure 5-13b).

We used Rayleigh circular statistics to analyze the distribution of SWR events relative to the phases of the respiratory cycle during resting conditions, i.e. with respiratory frequencies not exceeding $4 \mathrm{~Hz}$. This analysis revealed a significantly increased probability of SWR events during the early expiration phase of respiration (Rayleigh test: $\mathrm{n}=382 ; \mathrm{r}=0.14 ; \mathrm{z}=7.35 ; \mathrm{p}=0.02$ ). As illustrated in the polar plot in Figure 5-13c, the respiratory phase during which SWR probability was highest was around $210^{\circ}$, corresponding to the early expiration (Figure 5-13c).

\section{Effect of Inhibiting Respiration-Locked OB Activity on Respiration-Locked}

CA1 Activity. Using 4 mice with OBs treated with DREADDs vector we investigated the effect of respiration-locked OB activity on hippocampal LFP oscillations. Hippocampal LFP activity contains low-frequency oscillations in the delta and theta range that have been shown to be phase locked to nasal respiration ${ }^{189,190}$. Amplitudes of respirationaligned LFP activity in the hippocampus were compared immediately before and 30-min after CNO-injection in OB-DREADD treated mice. Respiratory and LFP activity was aligned on the end of expiration. The respiratory waveform was not changed by CNOinjection (Figure 5-14a). However, the respiration-aligned, average LFP signal shows a significant reduction in the amplitude of respiration-locked oscillations (Two-Sample ttest: $p<0.05$ ). Figure 5-14b shows an individual example. The bar-graph in Figure 5-14c represents the group data. 
a
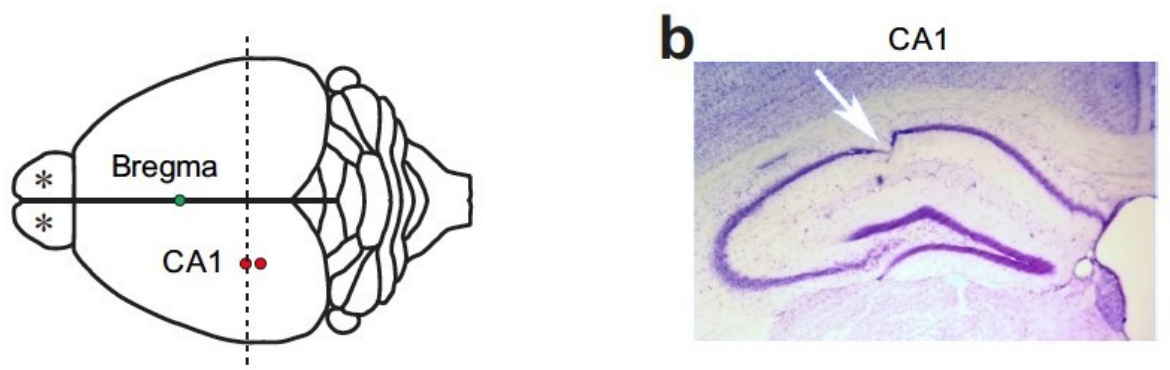

C

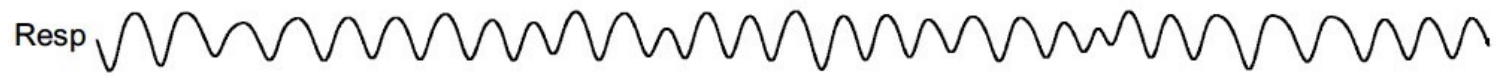

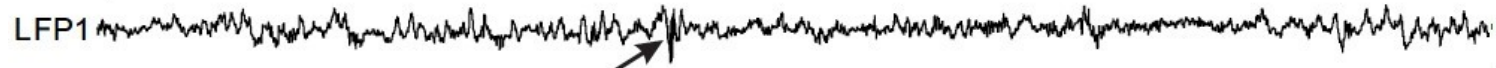
LFP2
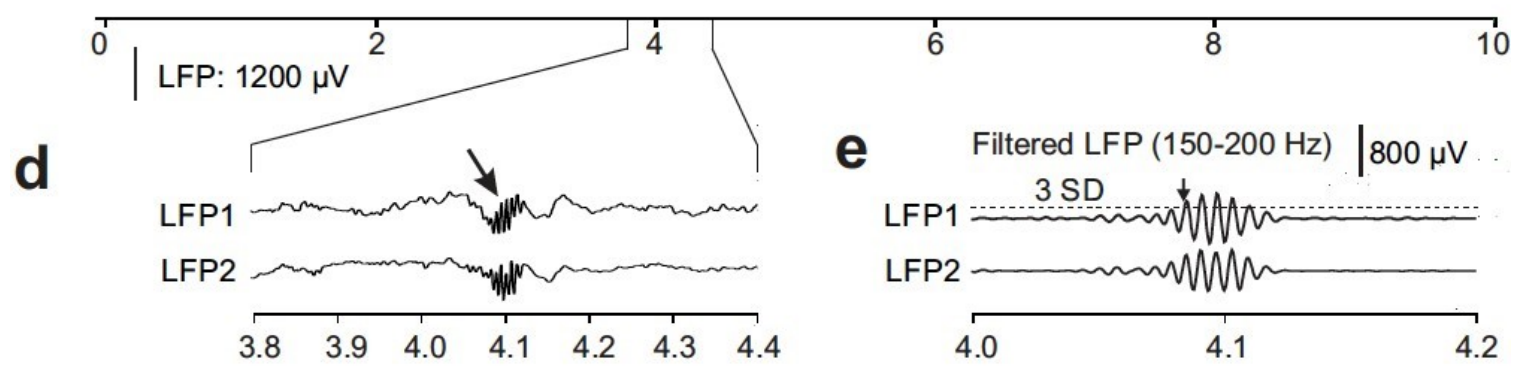

Figure 5-12. Recording sites and raw data examples of simultaneous recordings of local field potentials (LFPs) in the awake mouse.

(a) Schematic drawing of the top view of a mouse brain. LFPs were recorded from the left CA1 region of the hippocampus. Dashed line represents the coronal section for verifying the recording location in CA1. Stars represent sites of bilateral injections of DREADD vector in OB. (b) An example of an electrolytic lesion (arrow) in the CA1 region of hippocampus. (c) Examples of respiration (RESP) and raw LFP data. The RESP trace shows respiration related temperature changes with expiration causing an increase in temperature which corresponded to a decrease in voltage. LFP1 and LFP2 were recorded from electrodes in CA1. Arrow points at a characteristic high-frequency ripple activity. Abscissa represents time in seconds. (d) An enlarged view of raw LFPs around a hippocampal ripple event (arrow). Same LFP amplitude scale bar as in c. (e) High-pass filtered versions of the LFPs in panel d emphasizes the high-frequency ripple components of CA1 activity. Horizontal dashed line above LFP1 represents the mean filtered LFP amplitude plus 3 standard deviations (SD) from a continuous data of 60 seconds, which was used as a threshold (mean $\pm 3 \mathrm{SD}$ ) for automatic detection of sharp-wave ripple activity in the CA1 region. The arrow marks the beginning of ripple activity. 

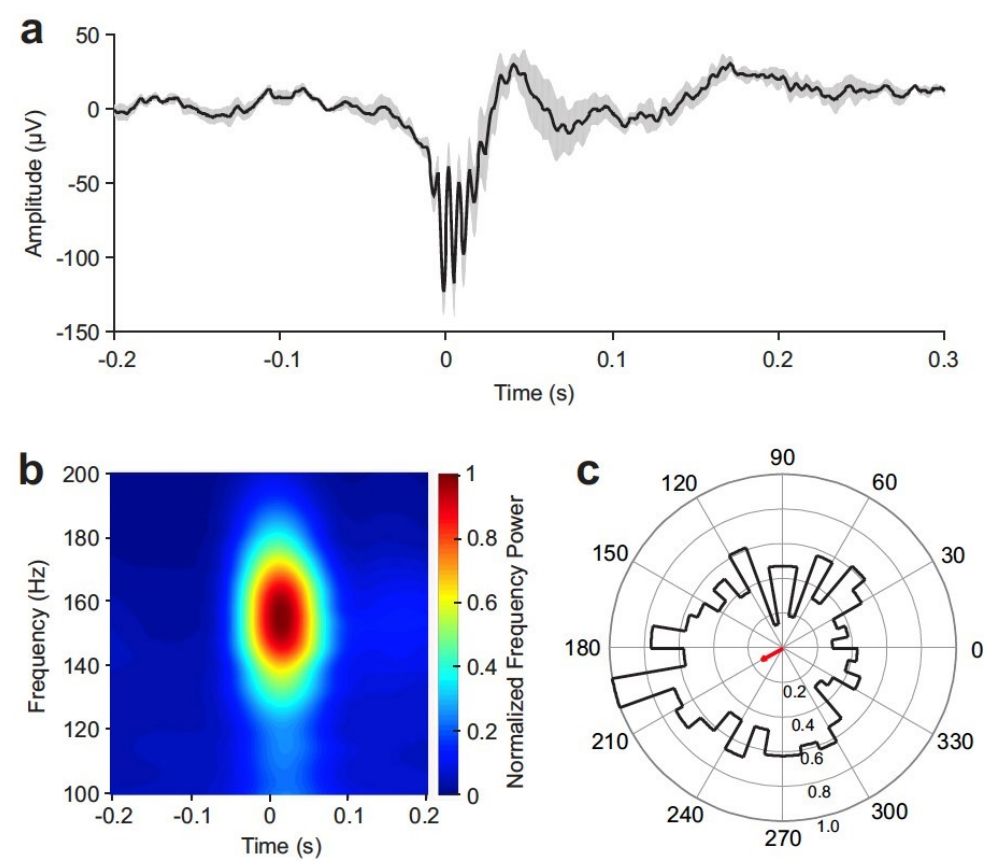

Figure 5-13. Hippocampal sharp-wave ripple (SWR) activity during the respiratory cycle in mice.

(a) Local field potential (LFP) around hippocampal SWRs (mean +/- standard error). Data are aligned on the onset of ripple-activity (at time $0 \mathrm{~s}$ ). (b) Time-Frequency mapping of LFPs around CA1 ripples. Color represents normalized frequency power. (c) Polar reflecting the distribution of SWR relative to respiratory phase. Red arrow represents the mean vector determined by circular statistics (Rayleigh test: $\mathrm{n}=382 ; \mathrm{r}=$ $0.14 ; \mathrm{z}=7.35 ; \mathrm{p}=0.02) .0^{\circ}$ represents the end of expiration, $180^{\circ}$ corresponds to the end of inspiration. Concentric circles mark $r$ values as indicated in the lower half of the circle. 

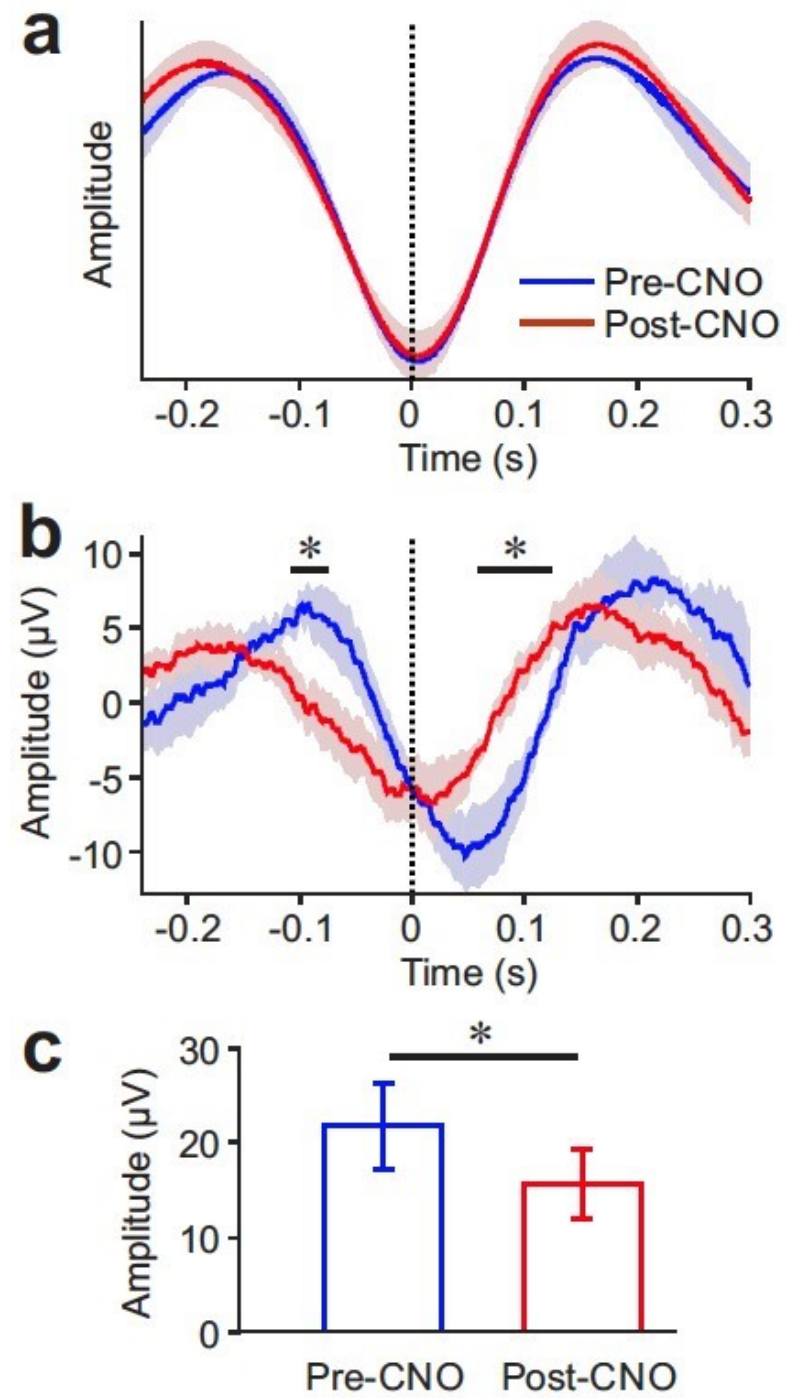

Figure 5-14. Changes in respiration and local field potential (LFP) recorded in hippocampal CA1 region following Clozapine $\mathrm{N}$-oxide (CNO) injection in DREADD-mice $(n=4)$.

(a) Average respiratory traces, showing identical breathing patterns before and after CNO-injection. (b) Top: Average LFP amplitude. Data are aligned on the end of expiration ( $0 \mathrm{~s})$. Same color code as in a. Two-Sample t-test: ${ }^{*} \mathrm{p}<0.05$. (c) Comparison of absolute amplitude change (maximum - minimum) of LFPs in respiratory cycle.

Paired-t-test: $* \mathrm{p}<0.05$. 
Effect of Respiration-Locked OB Activity SWR Event Timing. Finally, it was asked whether the SWR activity was influenced by respiration-locked OB activity. In the four DREADD treated mice the amplitudes, ripple frequency and the timing of SWR events relative to the phase of respiration were compared before and after inhibition of respiration-locked $\mathrm{OB}$ activity by $\mathrm{CNO}$ injection. In all four mice combined, a total of 289 and 310 SWR events were detected in comparable time periods before and $30 \mathrm{~min}$ after CNO injections, respectively. Inhibiting respiration-locked OB activity did not alter the amplitude of SWRs (Figure 5-15a). However, the power of the ripple oscillation measured with the $100-200 \mathrm{~Hz}$ frequency range was significantly reduced following CNO-injection (Figure 5-15b; Two-Sample t-test: $\mathrm{p}<0.05$ ). Analysis of SWR timing relative to the respiratory cycle prior to $\mathrm{CNO}$ injection revealed a significantly increased probability of SWR events during early expiration at a phase angle of around $210^{\circ}$

(Figure 5-15c; Rayleigh test: $r=0.18 ; z=9.73 ; p=0.001$ ). This is consistent with results from the control group of 5 mice that did not receive DREADD treatment. Analysis of measurements taken 30 min after CNO-injection no longer showed a significant relationship between respiratory phase and SWR events (Figure 5-15d; Rayleigh test: $r=$ $0.09 ; \mathrm{z}=2.53 ; \mathrm{p}=0.278)$.

\section{Discussion}

This study provides the first evidence that hippocampal SWR generation is modulated by respiration and that the influence of respiration on SWRs requires normal OB activity. The main new findings are that the probability of the hippocampal network to generate SWR activity is significantly increased during the early phase of the expiration, and that this modulation of SWR generation depends on an intact OB. Taking into account the slow buildup of excitatory activity that has been shown to precede the high-frequency ripple ${ }^{240}$, the phase of increased probability would be slightly advanced to late inspiration.

These findings also raise the question of how respiration-locked OB activity can modulate the probability of SWR generation. Current data suggest that SWRs are intrinsically generated within the hippocampal network ${ }^{241}$, as they are also generated in hippocampal slice preparations ${ }^{242,243}$. The probability of a SWR event in the intact brain could thus be modulated by respiration-locked activity reaching the hippocampus via the entorhinal cortex ${ }^{189,190}$. As demonstrated here (Figure 5-14) and previously by others $^{189,190}$, the effects of respiration-locked synaptic activity reaching the hippocampus via the entorhinal cortex can be directly observed in form of respiration-locked LFP oscillations. These results also show that loss of OB activity significantly reduces the amplitude of respiration-locked oscillations (Figure 5-14b). We hypothesize that the reduced modulation of hippocampal network excitability is no longer sufficient to cause SWR events generation to be phase-locked to respiration.

A second potential mechanism is less direct as it involves the suppression of SWR by cholinergic projections to the hippocampus from medial septal neurons ${ }^{244}$. As discussed earlier, the neurons in the basal forebrain nuclei, including cholinergic neurons, 

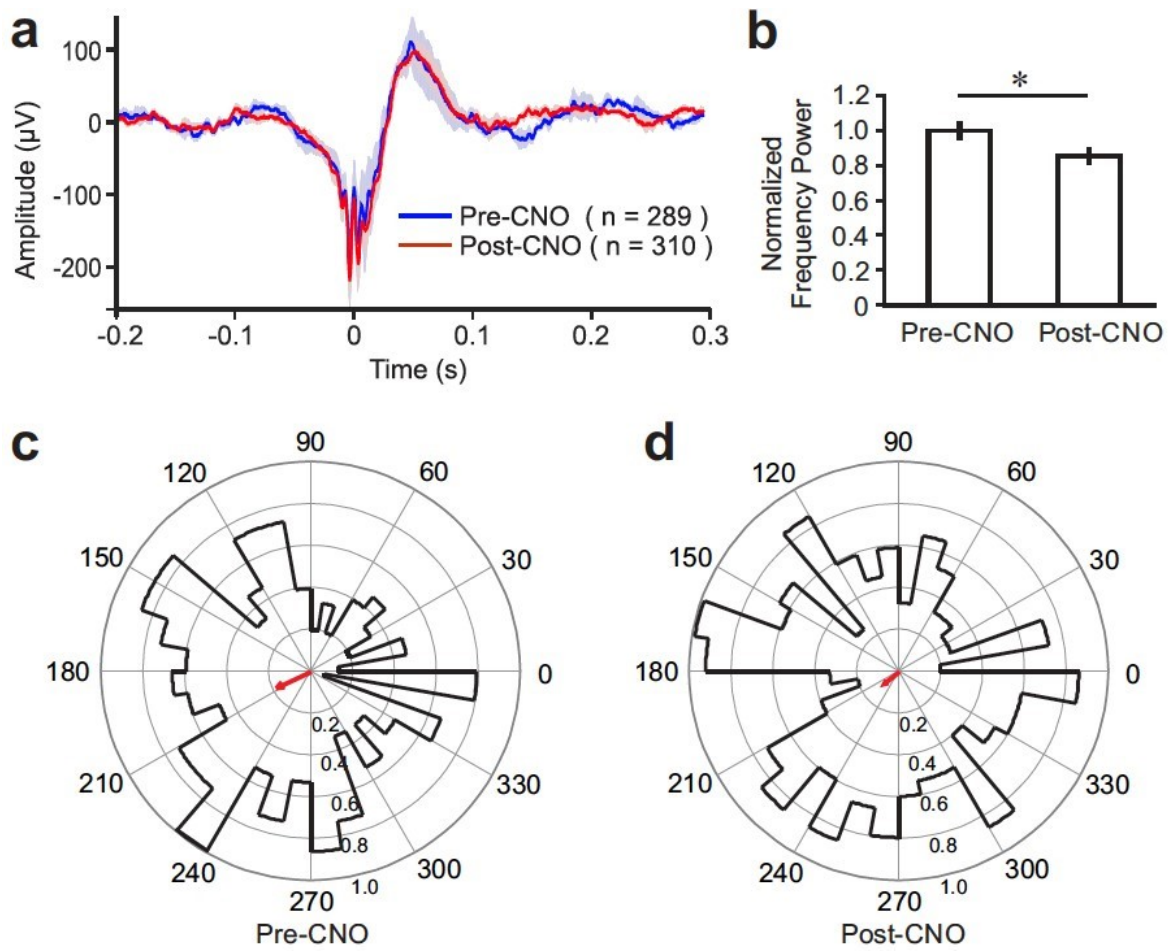

Figure 5-15. Changes in hippocampal sharp-wave ripple (SWR) activity before and after Clozapine N-oxide (CNO) Administration in mice with bilateral injections of DREADD vector in OB.

(a) Average LFP waveform aligned on the SWR onset $(t=0)$. Solid blue and red lines represents mean LFP traces pre and post CNO treatment, respectively. Shading represents standard error. (b) Comparison of normalized spectral power within the $100-200 \mathrm{~Hz}$ frequency band pre and post CNO treatment. Two-Sample t-test: ${ }^{*} \mathrm{p}<0.05$. (c) Polar coordinates $\left({ }^{\circ}\right)$ for SWR activities during respiratory cycles, showing normalized vectors (thicker line areas) and mean vector length (red arrow; $r$ ) determined by circular statistics (Rayleigh test). $0^{\circ}$ represents the end of expiration. The largest circle represents the maximum $r$ (1.0). Pre-CNO: $r=0.18 ; z=9.73 ; p=0.001 ;$ Post-CNO: $r=0.09 ; z=2.53$; $\mathrm{p}=0.278$. 
show rhythmic activity that is highly correlated with respiration-locked OB activity. Optogenetic activation of medial septal neurons, which provide cholinergic input to the hippocampus, has been shown to effectively suppress the generation of SWR in the hippocampal network ${ }^{244}$. A cholinergic input to the hippocampus that is modulated in phase with respiration could thus result in a rhythmic suppression of SWR and could create the respiration-locked bias observed here.

\section{Respiratory Modulation of Electrocorticography in Humans}

It is becoming clearer as more research groups become involved that rhythmic neuronal activity locked to respiration is an influential and far-reaching factor in the brain of rodents. These model organisms contribute irreplaceably to our understanding of neurophysiology, so understanding the effects of respiration provides essential context for the interpretation of virtually any finding based on in vivo electrophysiology.

Does respiration provide a similar fundamental rhythm in human brain function? There are certainly reasons to be skeptical. Most importantly, the relative size of the olfactory bulbs relative to the neocortex is quite different in rodents and humans, and rodents are obligate nasal breathers, making it less likely that there is a large and persistent effect of respiration in humans that is mediated by olfactory bulb output.

Conversely, there are also good reasons to speculate that respiration acts as an important modulator of brain activity in humans. Perhaps the most self-evident reason is that humans have evolved to modify their respiration to produce meaningful speech, a function that engages sizeable portions of the neocortex in a coordinated fashion ${ }^{245}$. Speech necessarily requires an accurate internal representation of respiratory phase, and studies of breathing patterns in subjects listening to speech show breathing changes ${ }^{246}$, suggesting a codependence of respiratory function and information processing related to speech. Additionally, human subjects report experiencing emotional changes related to deep intentional breathing, and perform better on difficult tasks during breath holding. While many of these effects of respiration are subjective, quality of life has in some cases been improved by regular breathing exercises, including in a group of veterans suffering from anxiety and post-traumatic stress disorder ${ }^{247}$. How these specific effects are related to respiration-locked oscillations in the brain is not yet well understood.

Here I present findings related to respiration-locked oscillations in the human brain based on clinical ECoG recordings from anonymous male and female human subjects between the ages of 12 and 28 . Respiration was measured using a stretchsensitive belt positioned around the lower rib cage. Patients were typically at rest, sometimes asleep or on the verge of sleep. Respiration phases were normalized within intervals between peaks in tidal volume. Correspondence between electrode recordings and anatomical location was provided via $3 \mathrm{D}$ projection onto a template brain using MRI and CT images. 


\section{Respiration-Locked Slow Oscillations}

Identification of respiration-locked slow oscillations was a primary investigative goal, thus ECoG waveform averages were calculated for peak moments in tidal volume. High amplitude slow oscillations aligned with respiration were rare within the group, although a few examples of outstanding modulation were statistically evaluated using a bootstrap method. Figure 5-16 shows results from a patient with fairly broad modulation that proved to be statistically significant. Cortical areas with significant modulation were found in the superior frontal, inferior frontal, temporal and parietal lobes, suggesting a widespread entrainment of neuronal activity to respiration while the patient was at rest.

\section{Amplitude Coupling of Fast ECoG Oscillations to the Respiratory Phase}

Secondarily, PAC of ECoG oscillation amplitudes to the respiratory phase was assessed using the Moore-Rayleigh approach detailed in Chapter 2. In each subject, this analysis was executed on activity in 23 frequency bands from each recording site to determine whether oscillatory activity was significantly modulated in phase with respiration, and whether the significant results were anatomically clustered.

Respiration PAC was more frequently identified than slow oscillations, with significant gamma-band effects in 11 of 13 patients. The most common frequency range of modulation was between 64 and $96 \mathrm{~Hz}$. Figure 5-17 shows example results from three patients with different modulation profiles. Modulation was typically found to be anatomically broad, yet some patients had stronger modulation within focal regions.

\section{Discussion and Conclusion}

Given this new evidence, it appears that respiration locked neuronal oscillations do occur in the human brain. Respiration-frequency oscillations were less prominent in the human, although this may be a consequence of the lower respiratory rate, and the technical capability to capture slow oscillations. The lowest frequency low-cut filter setting in the clinical ECoG equipment was $0.1 \mathrm{~Hz}$, which may have diminished some slow electrical activity. Respiration PAC was much more reliably captured, especially for oscillations in the gamma range between 64 and $96 \mathrm{~Hz}$. Adjacent clusters of electrodes with significant modulation were also observed around $4 \mathrm{~Hz}$ and $12-16 \mathrm{~Hz}$ in multiple patients.

Meaningful localization of cluster trends across patients was not feasible in this context, given that the primary purpose of electrode implantation was to map pathological tissue relative to eloquent cortex. For example, of the eleven grids implanted upon a unilateral portion of the cortex, nine of them were implanted on the left side. Of those on the left, virtually all covered inferior frontal and superior temporal regions, many of which were activated by speech. While this is consistent with the hypothesis that respiration locked oscillations influence speech-related areas of the cortex, identification 

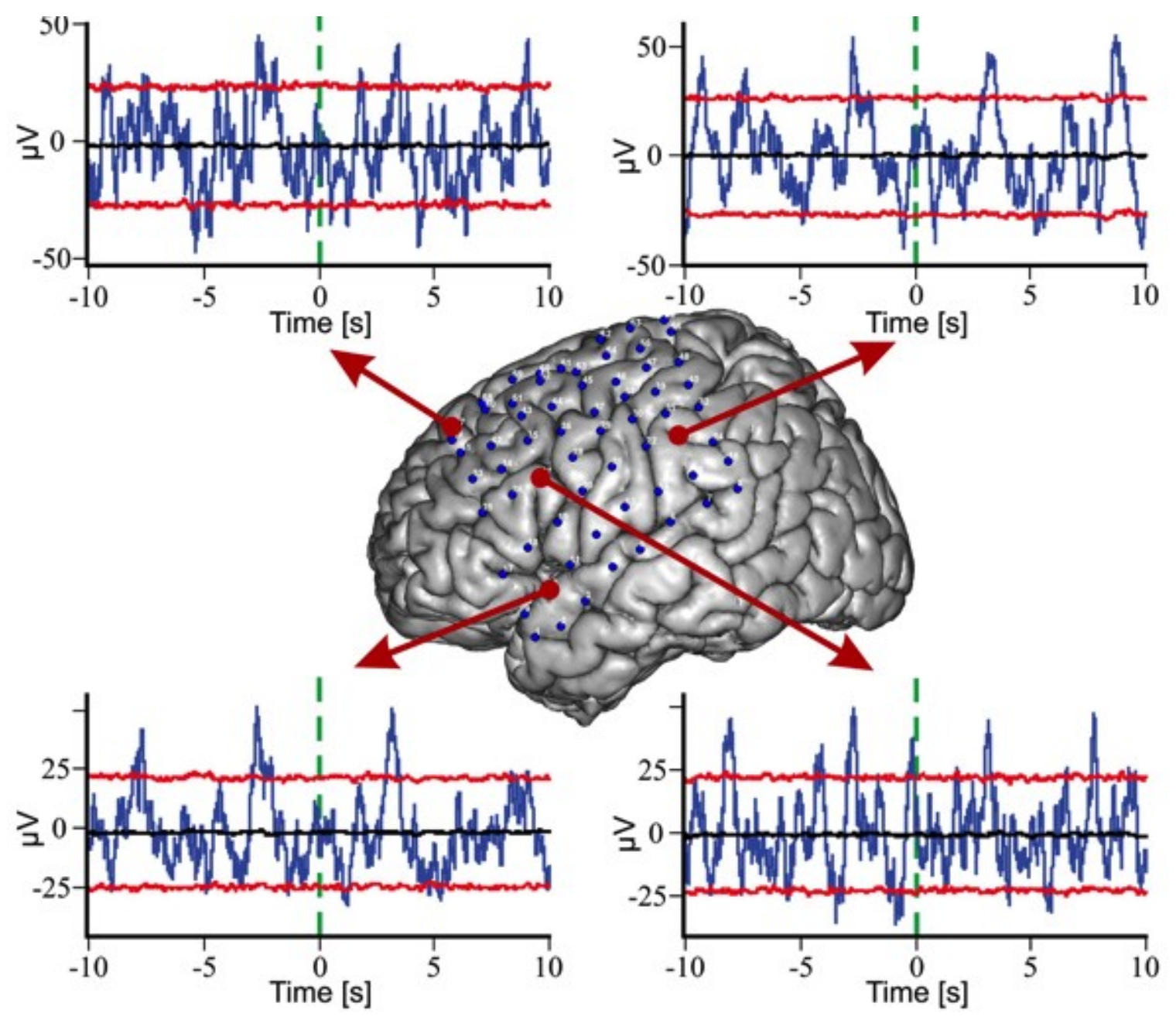

Figure 5-16. Slow respiration-locked cortical oscillations in the human brain, as measured by surface platinum electrode grid.

Purple traces show electrocorticographic (ECoG) activity averages aligned on the end of inspiration, corresponding to the electrode locations at arrow origins for one patient. Red lines represent $99 \%$ statistical boundaries determined by time-shuffled bootstrap analysis. Significantly large respiration-locked slow oscillations appear in all recorded cortical regions, including superior frontal, inferior frontal, superior temporal, and anterior parietal areas. 
A

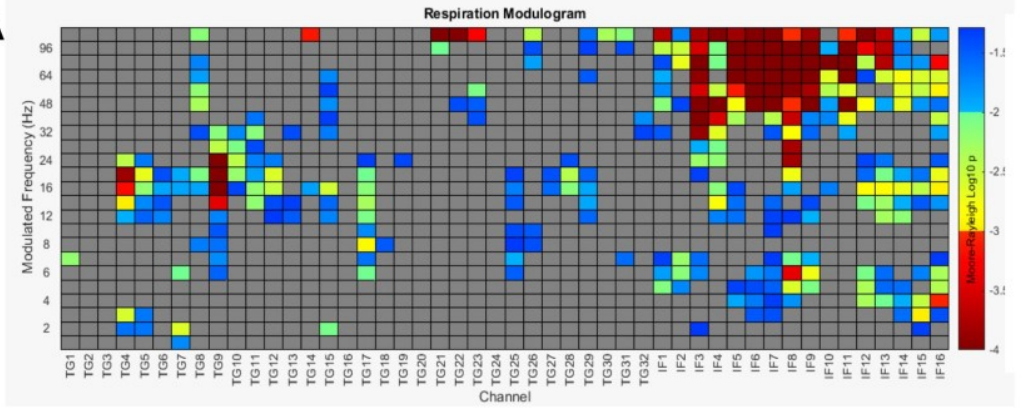

C

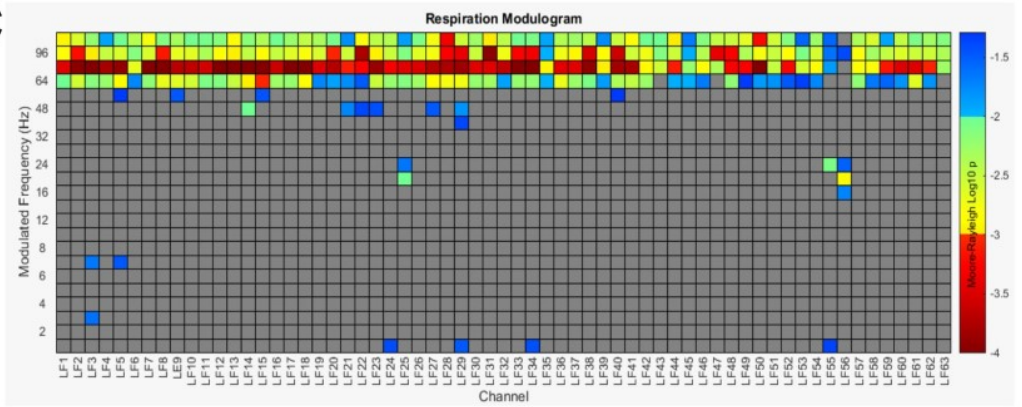

E

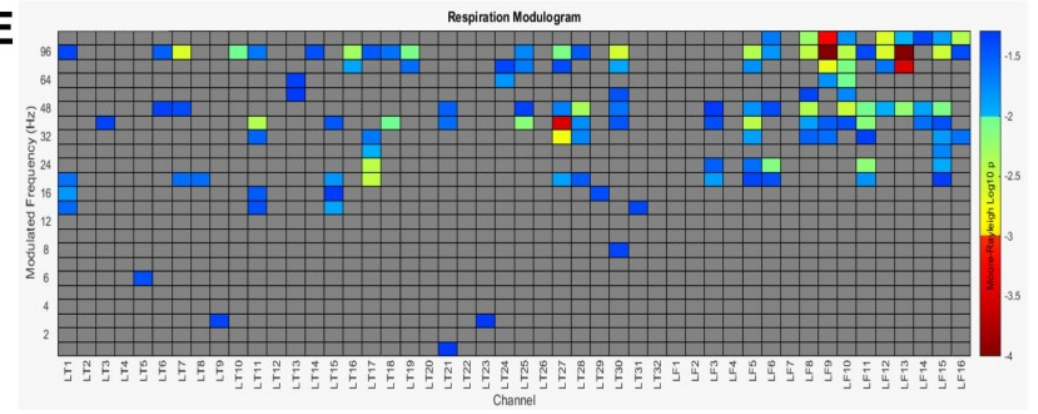

B

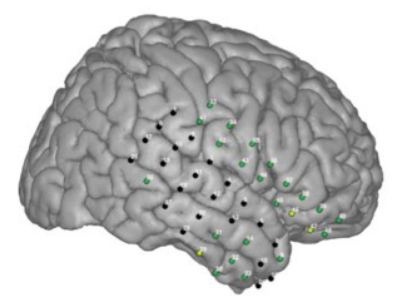

D

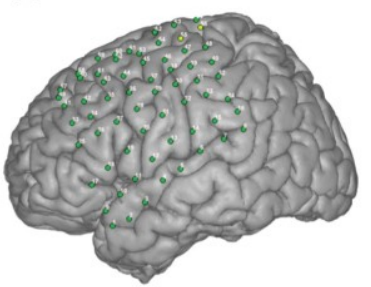

$\mathbf{F}$

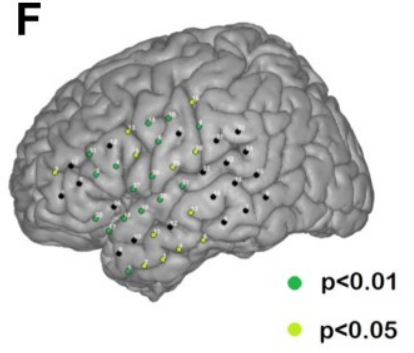

Figure 5-17. Respiration PAC statistical analysis for recording grids in 3 patients, and corresponding grid locations for significant gamma PAC.

Columns in resulting plot correspond to unique electrodes in the arrays. Rows indicate frequency bands. Gray boxes are not significant, blue are $p<0.05$, green $p<0.01$, red $\mathrm{p}<0.001$, and maroon $\mathrm{p}<0.0001$. Black electrode locations have no significant respirationgamma PAC, light green is $p<0.05$ and dark green is $p<0.01$. (A, B) Example of patient with focal respiration-gamma PAC, present on inferior frontal electrodes, but not temporal. (C,D) Example of patient with broad respiration-gamma PAC between 60 and $90 \mathrm{~Hz}$. This example is the same patient as in Figure 5-16, exhibiting both broad slow respiration-locked oscillations and respiration-gamma PAC. (E, F) Example of patient with relatively weak and sparse respiration PAC. Significant respiration-gamma PAC appears to be clustered near the center of the grid, and is strongest along the lateral sulcus, on the superior temporal and inferior frontal gyri. 
of consistent respiration modulation throughout these recordings does not lead to the conclusion that respiration PAC is a truly global phenomenon.

Nevertheless, what could respiration PAC in the cortex mean for information processing in general? The question of whether or not the rhythmic input from respiration carries meaningful sensory content remains unresolved, but the effect shown in the LFP suggests that respiration at least plays an important role in state changes in the network related to information processing. This is further supported by the finding that the occurrence of phase transitions (also described as "beats" in Chapter 1) is biased towards certain phases of respiration ${ }^{248}$. These phase transitions are thought to be an essential macroscopic feature of information processing by the brain ${ }^{249}$, and are correlated in time with moments of conscious stimulus categorization ${ }^{250}$. Phase transitions can be simulated in interconnected layers of cortical models ${ }^{250,251}$, and in such models represent transient episodes of inter-areal synchronization. These transitions take place because the network model remains in a meta-stable state (similar to water at boiling temperature) between unrestrained global oscillation and unrealistically sparse activity. In this state, oscillations occur randomly and in an unstable manner until a small outside influence drives its target toward one state or the other, with excitatory input inducing a temporarily stable oscillation $^{251}$. The effect of a slow excitatory rhythmic input similar to respiration has been simulated in these models, resulting in broad slow oscillation, broad phaseamplitude coupling, and phase transition patterns that are all temporally aligned with the input $^{197,248}$.

Being able to break in and out of a stable oscillatory state is essential for encoding and transmitting information in a flexible manner ${ }^{13,81,249}$, and the evidence thus far suggests that respiration-locked sensory input has an influence on when transitions between these two states occur. Thus, if phase transitions are truly emblematic of information encoding, one would expect respiration to influence measurable variables related to encoding, such as reaction time. Indeed, reaction times in a visual discrimination task were shown to be longer in human subjects during oral breathing than during nasal breathing, and longer during oral inspiration than oral expiration ${ }^{252}$. Furthermore, they reported an amplitude modulation of oscillations in the beta and gamma ranges in phase with nasal respiration ${ }^{252}$. Altogether, this evidence suggests that sensory input from nasal breathing may provide nonspecific excitatory input which serves to bring the cortex closer to the critical state for a phase transition, allowing the neuronal activity related to perception to form more efficiently. As such, the particular frequencies that appear to be amplitude-modulated by respiration would be determined by network and cellular properties that generate oscillations during sensory processing, which may explain the variability in the frequency of respiration PAC observed here.

While it is reassuring that similar patterns are observed in humans and model organisms, respiration-locked oscillations in the human brain likely have unique and important functions that will have to be elucidated through further studies in human subjects, which may go further in linking these patterns to specific brain functions by pursuing non-invasive approaches. Magnetoencephalography has reportedly proven useful for capturing oscillations with frequencies up to $60^{253}$ or $80 \mathrm{~Hz}^{254}$, which would be 
suitable to detect many of these rhythms. Furthermore, cognitive tasks involving language or other demands could be performed while brain activity and respiration are measured, allowing for a better understanding of the functional relevance of respirationlocked activity for meaningful cortical computation. 


\section{CHAPTER 6. SUMMARY AND CONCLUSIONS}

This work was undertaken to develop and fully justify methods of analysis which are optimized for specific applications in the neurosciences, with the notion that the most pertinent and efficient possible methods of analysis lead to the most intelligible results, and ultimately the clearest insights into brain function.

The foundation of these studies and methods of analysis is the communication through coherence hypothesis, the underpinnings of which were summarized in Chapter 1. The main thrust of this hypothesis is that synchronized oscillatory activity in communicating sub-networks within the brain allows for effective communication between the neurons within them. Thus, being able to measure oscillatory activity and quantify its synchrony across brain areas should allow for an assessment on neuronal communication and brain function.

The concept of phase synchronization in the brain has remained closely aligned with the CTC hypothesis, and even predates its formal articulation by several years ${ }^{12}$. This concept differs mostly in its methodology; it is characterized by the modeling of network output as the manifestation of a spectrum of oscillators, for which phase can be instantaneously defined, and for which synchronization behavior with other oscillators is well described. This manner of thinking about brain function creates many new possibilities in terms of technical analysis and provides a clarifying lens for the interpretation of electrical brain data.

In Chapter 3, new findings were presented relating synchronous oscillations in the visual thalamus and cortex of the mouse to the precise synchronization of neuronal firing in both regions. In order to relate the synchronous firing of these neurons to extrinsic oscillations in the network, I developed a novel method of quantifying changes in precise synchrony in a time-resolved manner based on prior knowledge about the latency of transmission and the time constant for excitatory input in the target (Figure 2-7). Previous efforts to investigate the pulsatile nature of transmission relative to stimulusevoked network activity depended on arbitrary binning in the phase domain ${ }^{255}$. The novel approach is consistent with established standards in spike jittering analyses, and furthermore allows for correlation analysis with different frequencies of oscillatory network activity in order to identify those consistently related to inter-areal communication (Figure 3-5).

In a related study within Chapter 5, a novel phase synchronization analysis (Figures 2-4, 2-5, and 2-6) was used in order to detect synchronization patterns that were unique to the stimulus. This allowed for the quantification of the effect of a stimulus on synchronization, and a distinction between normal stimulus-evoked synchronization and synchronization after OB inhibition (Figure 5-11). The disruption of synchronization and visual perception by $\mathrm{OB}$ inhibition identifies a possible role for the $\mathrm{OB}$ in maintaining the global state of the brain, a similar effect that locomotion was recently found to have 220,222 . Such a test for synchronization could become very useful to the systems neuroscience 
community, as it is more sensitive to stimulus-evoked changes than conventional analyses (Figure 2-5b), and allows for statistical estimation of synchrony changes that is more efficient than any previous method, making it easier to identify frequencies and latencies at which outstanding changes in synchronization take place (Figure 2-5d).

In Chapter 4, the question of how cerebellar activity is involved in spatial working memory was posed. The hypothesis was developed that cerebellar activity plays an ancillary role in processing related to memory by engaging in what it is thought to do best: integrating input and making a timing judgment ${ }^{161,181}$. The possibility was considered that neuronal output from the cerebellum reflected the relative timing of neuronal oscillations in prefrontal cortex and dorsal hippocampus related to memory function. Testing this possibility required simultaneous recordings from three regions of the brain as well as a novel approach to interpret ternary neuronal relationships. This led me to develop the method of phase relationship tuning (Figure 2-9), with which patterns of interactions at various frequencies were identified (Figure 4-3). Perhaps most interesting was the relationship between Purkinje cell firing rate and the PFC-CA1 theta rhythm relationship, which has been linked to spatial working memory ${ }^{256}$.

Finally in Chapter 5, various methods of analysis for oscillatory network behavior were used to identify cases in which the respiratory cycle modulates brain activity. First in rodents, I was able to develop a highly effective method of measurement for respiration, and identify some previously unknown brain regions within which neuronal rhythms are structured around the breath cycle. Perhaps most important among these findings is the dependence of basal forebrain oscillations on olfactory bulb output, as the basal forebrain contributes critically to cortical cholinergic tone and global brain state ${ }^{192}$. This could explain changes observed in visual system function after OB inhibition. Given that $\mathrm{BF}$ activation enhances visual processing ${ }^{221}$, inactivation or disruption of normal BF activity is likely to have the inverse effect.

Similar relationships between respiration and brain oscillations were identified in human data as well, acquired from electrode grids placed on the cortical surface to monitor epileptiform activity. The most substantial finding was that of gamma oscillation amplitude coupling to the respiration phase, a phenomenon that was identified in nearly $85 \%$ of the patients evaluated. This finding relied on a novel phase-amplitude coupling analysis, which allows for a relatively fast statistical estimation without the burden of generating surrogates. This allowed for the analysis of long stretches of data - typically greater than 15 minutes at greater than $1 \mathrm{kHz}$ sampling - from hundreds of electrodes, across several frequency bands with only a few weeks of computation on a desktop computer. Needless to say, this investigation would not have been feasible with the tools at hand had it taken more than two hundred times longer to generate surrogates. In any case, the finding that gamma oscillations are modulated by respiration in human cortex should inspire further investigation into how specific brain functions might be affected, especially those intimately related to respiration, like language processing.

Given the diversity of the studies undertaken, it would not be fitting to impose a unifying conclusion about the specific results herein. Rather, I hope to have illuminated 
new possible approaches for basic research via the observation and analysis of neuronal oscillations, having demonstrated new methods that address a range of questions, from small ones concerning inter-regional communication among individual neurons to questions about large-scale integration in memory networks. 


\section{LIST OF REFERENCES}

$1 \quad$ von der Malsburg, C. in Models of Neural Networks: Temporal Aspects of Coding and Information Processing in Biological Systems (eds Eytan Domany, J. Leo van Hemmen, \& Klaus Schulten) 95-119 (Springer New York, 1994).

2 Hebb, D. O., Martinez, J. L. \& Glickman, S. E. The Organization of Behavior - a Neuropsychological Theory - Hebb,Do. Contemp Psychol 39, 1018-1020 (1994).

3 Gray, C. M., Konig, P., Engel, A. K. \& Singer, W. Oscillatory responses in cat visual cortex exhibit inter-columnar synchronization which reflects global stimulus properties. Nature 338, 334-337, doi:10.1038/338334a0 (1989).

4 Gray, C. M. \& Singer, W. Stimulus-specific neuronal oscillations in orientation columns of cat visual cortex. Proc Natl Acad Sci U S A 86, 1698-1702 (1989).

5 Hubel, D. H. \& Wiesel, T. N. Receptive fields, binocular interaction and functional architecture in the cat's visual cortex. J Physiol 160, 106-154 (1962).

6 Hubel, D. H. \& Wiesel, T. N. Receptive fields of single neurones in the cat's striate cortex. J Physiol 148, 574-591 (1959).

7 Ahn, S. M. \& Freeman, W. J. Steady-state and limit cycle activity of mass of neurons forming simple feedback loops (I): lumped circuit model. Kybernetik 16, 87-91 (1974).

8 Freeman, W. J. Nonlinear dynamics of paleocortex manifested in the olfactory EEG. Biol Cybern 35, 21-37 (1979).

9 Bressler, S. L. \& Freeman, W. J. Frequency analysis of olfactory system EEG in cat, rabbit, and rat. Electroencephalogr Clin Neurophysiol 50, 19-24 (1980).

10 Boudreau, J. C. \& Freeman, W. J. Spectral analysis of electrical activity in the prepyriform cortex of the cat. Experimental Neurology 8, 423-439, doi:http://dx.doi.org/10.1016/0014-4886(63)90064-5 (1963).

11 Engel, A. K., Fries, P. \& Singer, W. Dynamic predictions: oscillations and synchrony in top-down processing. Nat Rev Neurosci 2, 704-716, doi:10.1038/35094565 (2001).

12 Varela, F., Lachaux, J. P., Rodriguez, E. \& Martinerie, J. The brainweb: phase synchronization and large-scale integration. Nat Rev Neurosci 2, 229-239, doi:10.1038/35067550 (2001).

13 Friston, K. J., Bastos, A. M., Pinotsis, D. \& Litvak, V. LFP and oscillations-what do they tell us? Curr Opin Neurobiol 31, 1-6, doi:10.1016/j.conb.2014.05.004 (2015). 
14 Fries, P. A mechanism for cognitive dynamics: neuronal communication through neuronal coherence. Trends Cogn Sci. 9, 474-480 (2005).

15 Volgushev, M., Chistiakova, M. \& Singer, W. Modification of discharge patterns of neocortical neurons by induced oscillations of the membrane potential. Neuroscience 83, 15-25 (1998).

16 Fries, P. Rhythms for Cognition: Communication through Coherence. Neuron 88, 220-235, doi:10.1016/j.neuron.2015.09.034 (2015).

17 Colgin, L. L. et al. Frequency of gamma oscillations routes flow of information in the hippocampus. Nature 462, 353-357, doi:10.1038/nature08573 (2009).

18 Bastos, A. M., Briggs, F., Alitto, H. J., Mangun, G. R. \& Usrey, W. M. Simultaneous recordings from the primary visual cortex and lateral geniculate nucleus reveal rhythmic interactions and a cortical source for gamma-band oscillations. J Neurosci 34, 7639-7644, doi:10.1523/JNEUROSCI.4216-13.2014 (2014).

19 Bastos, A. M. et al. Visual areas exert feedforward and feedback influences through distinct frequency channels. Neuron 85, 390-401, doi:10.1016/j.neuron.2014.12.018 (2015).

20 Richter, C. G., Thompson, W. H., Bosman, C. A. \& Fries, P. Top-down beta enhances bottom-up gamma. J Neurosci, doi:10.1523/JNEUROSCI.3771-16.2017 (2017).

21 Brette, R. Philosophy of the Spike: Rate-Based vs. Spike-Based Theories of the Brain. Front Syst Neurosci 9, 151, doi:10.3389/fnsys.2015.00151 (2015).

22 Buzsaki, G., Anastassiou, C. A. \& Koch, C. The origin of extracellular fields and currents--EEG, ECoG, LFP and spikes. Nat Rev Neurosci 13, 407-420, doi:10.1038/nrn3241 (2012).

23 Humphrey, D. R. \& Schmidt, E. M. in Neurophysiological Techniques: Applications to Neural Systems (eds Alan A. Boulton, Glen B. Baker, \& Case H. Vanderwolf) 1-64 (Humana Press, 1990).

24 Harris, K. D., Henze, D. A., Csicsvari, J., Hirase, H. \& Buzsaki, G. Accuracy of tetrode spike separation as determined by simultaneous intracellular and extracellular measurements. J Neurophysiol 84, 401-414 (2000).

25 Wood, F., Black, M. J., Vargas-Irwin, C., Fellows, M. \& Donoghue, J. P. On the variability of manual spike sorting. IEEE Trans Biomed Eng 51, 912-918, doi:10.1109/TBME.2004.826677 (2004).

26 Margulis, M. \& Tang, C. M. Temporal integration can readily switch between sublinear and supralinear summation. J Neurophysiol 79, 2809-2813 (1998). 
27 Softky, W. Sub-millisecond coincidence detection in active dendritic trees. Neuroscience 58, 13-41 (1994).

28 Azouz, R. \& Gray, C. M. Dynamic spike threshold reveals a mechanism for synaptic coincidence detection in cortical neurons in vivo. Proc Natl Acad Sci U S A 97, 8110-8115, doi:10.1073/pnas.130200797 (2000).

29 Higgs, M. H. \& Spain, W. J. Kv1 channels control spike threshold dynamics and spike timing in cortical pyramidal neurones. $J$ Physiol 589, 5125-5142, doi:10.1113/jphysiol.2011.216721 (2011).

30 Leger, J. F., Stern, E. A., Aertsen, A. \& Heck, D. Synaptic integration in rat frontal cortex shaped by network activity. J Neurophysiol 93, 281-293, doi:10.1152/jn.00067.2003 (2005).

31 Hutcheon, B. \& Yarom, Y. Resonance, oscillation and the intrinsic frequency preferences of neurons. Trends Neurosci 23, 216-222 (2000).

32 Tiesinga, P. H., Fellous, J. M., Salinas, E., Jose, J. V. \& Sejnowski, T. J. Inhibitory synchrony as a mechanism for attentional gain modulation. J Physiol Paris 98, 296-314, doi:10.1016/j.jphysparis.2005.09.002 (2004).

33 Moca, V. V., Nikolic, D., Singer, W. \& Muresan, R. C. Membrane resonance enables stable and robust gamma oscillations. Cerebral cortex 24, 119-142, doi:10.1093/cercor/bhs293 (2014).

34 Walter, W. G. Critical Review: The Technique and Application of ElectroEncephalography. J Neurol Psychiatry 1, 359-385 (1938).

35 Sanchez-Vives, M. V. \& McCormick, D. A. Cellular and network mechanisms of rhythmic recurrent activity in neocortex. Nat Neurosci 3, 1027-1034, doi:10.1038/79848 (2000).

36 Gloor, P., Ball, G. \& Schaul, N. Brain lesions that produce delta waves in the EEG. Neurology 27, 326-333 (1977).

37 Steriade, M., Gloor, P., Llinas, R. R., Lopes de Silva, F. H. \& Mesulam, M. M. Report of IFCN Committee on Basic Mechanisms. Basic mechanisms of cerebral rhythmic activities. Electroencephalogr Clin Neurophysiol 76, 481-508 (1990).

38 Sirota, A. \& Buzsaki, G. Interaction between neocortical and hippocampal networks via slow oscillations. Thalamus Relat Syst 3, 245-259, doi:10.1017/S1472928807000258 (2005).

39 Sejnowski, T. J. \& Destexhe, A. Why do we sleep? Brain Res 886, 208-223 (2000). 
40 Walker, M. P. The role of slow wave sleep in memory processing. J Clin Sleep Med 5, S20-26 (2009).

41 Staresina, B. P. et al. Hierarchical nesting of slow oscillations, spindles and ripples in the human hippocampus during sleep. Nat Neurosci 18, 1679-1686, doi:10.1038/nn.4119 (2015).

42 Petsche, H., Stumpf, C. \& Gogolak, G. [The significance of the rabbit's septum as a relay station between the midbrain and the hippocampus. I. The control of hippocampus arousal activity by the septum cells]. Electroencephalogr Clin Neurophysiol 14, 202-211 (1962).

43 Hasselmo, M. E. \& Stern, C. E. Theta rhythm and the encoding and retrieval of space and time. Neuroimage 85 Pt 2, 656-666, doi:10.1016/j.neuroimage.2013.06.022 (2014).

44 Canolty, R. T. \& Knight, R. T. The functional role of cross-frequency coupling. Trends Cogn Sci 14, 506-515, doi:10.1016/j.tics.2010.09.001 (2010).

45 Denker, M. et al. The local field potential reflects surplus spike synchrony. Cerebral cortex 21, 2681-2695, doi:10.1093/cercor/bhr040 (2011).

46 Mainen, Z. F. \& Sejnowski, T. J. Reliability of spike timing in neocortical neurons. Science 268, 1503-1506 (1995).

47 Freeman, W. J. \& Kozma, R. Freeman's mass action. Scholarpedia 5, 8040 (2010).

48 Baker, S. N. Oscillatory interactions between sensorimotor cortex and the periphery. Curr Opin Neurobiol 17, 649-655, doi:10.1016/j.conb.2008.01.007 (2007).

49 Roopun, A. K. et al. A beta2-frequency $(20-30 \mathrm{~Hz})$ oscillation in nonsynaptic networks of somatosensory cortex. Proc Natl Acad Sci U S A 103, 15646-15650, doi:10.1073/pnas.0607443103 (2006).

50 Wetmore, D. Z. \& Baker, S. N. Post-spike distance-to-threshold trajectories of neurones in monkey motor cortex. J Physiol 555, 831-850, doi:10.1113/jphysiol.2003.048918 (2004).

51 Little, S. \& Brown, P. The functional role of beta oscillations in Parkinson's disease. Parkinsonism Relat Disord 20 Suppl 1, S44-48, doi:10.1016/S13538020(13)70013-0 (2014).

52 Kay, L. M. Circuit oscillations in odor perception and memory. Progress in brain research 208, 223-251, doi:10.1016/B978-0-444-63350-7.00009-7 (2014). 
53 Ito, J., Maldonado, P., Singer, W. \& Grun, S. Saccade-related modulations of neuronal excitability support synchrony of visually elicited spikes. Cerebral cortex 21, 2482-2497, doi:10.1093/cercor/bhr020 (2011).

54 Buzsaki, G. \& Wang, X. J. Mechanisms of gamma oscillations. Annu Rev Neurosci 35, 203-225, doi:10.1146/annurev-neuro-062111-150444 (2012).

55 Gregoriou, G. G., Gotts, S. J., Zhou, H. \& Desimone, R. High-frequency, longrange coupling between prefrontal and visual cortex during attention. Science 324, 1207-1210, doi:10.1126/science.1171402 (2009).

56 Brown, P. Cortical drives to human muscle: the Piper and related rhythms. Progress in neurobiology 60, 97-108 (2000).

57 Polania, R., Krajbich, I., Grueschow, M. \& Ruff, C. C. Neural oscillations and synchronization differentially support evidence accumulation in perceptual and value-based decision making. Neuron 82, 709-720, doi:10.1016/j.neuron.2014.03.014 (2014).

58 Nyhus, E. \& Curran, T. Functional role of gamma and theta oscillations in episodic memory. Neuroscience and biobehavioral reviews 34, 1023-1035, doi:10.1016/j.neubiorev.2009.12.014 (2010).

59 Engelhard, B. \& Vaadia, E. Spatial computation with gamma oscillations. Front Syst Neurosci 8, 165, doi:10.3389/fnsys.2014.00165 (2014).

60 Buzsaki, G. \& Moser, E. I. Memory, navigation and theta rhythm in the hippocampal-entorhinal system. Nat Neurosci 16, 130-138, doi:10.1038/nn.3304 (2013).

61 Fries, P., Nikolic, D. \& Singer, W. The gamma cycle. Trends Neurosci 30, 309316, doi:10.1016/j.tins.2007.05.005 (2007).

62 Burchell, T. R., Faulkner, H. J. \& Whittington, M. A. Gamma frequency oscillations gate temporally coded afferent inputs in the rat hippocampal slice. Neuroscience letters 255, 151-154 (1998).

63 Cardin, J. A. et al. Driving fast-spiking cells induces gamma rhythm and controls sensory responses. Nature 459, 663-667, doi:10.1038/nature08002 (2009).

64 Farrant, M. \& Nusser, Z. Variations on an inhibitory theme: phasic and tonic activation of GABA(A) receptors. Nat Rev Neurosci 6, 215-229, doi:10.1038/nrn1625 (2005).

65 Bartos, M., Vida, I. \& Jonas, P. Synaptic mechanisms of synchronized gamma oscillations in inhibitory interneuron networks. Nat Rev Neurosci 8, 45-56, doi:10.1038/nrn2044 (2007). 
66 Ni, J. et al. Gamma-Rhythmic Gain Modulation. Neuron 92, 240-251, doi:10.1016/j.neuron.2016.09.003 (2016).

67 Ray, S. \& Maunsell, J. H. Differences in gamma frequencies across visual cortex restrict their possible use in computation. Neuron 67, 885-896, doi:10.1016/j.neuron.2010.08.004 (2010).

68 Merker, B. Cortical gamma oscillations: the functional key is activation, not cognition. Neuroscience and biobehavioral reviews 37, 401-417, doi:10.1016/j.neubiorev.2013.01.013 (2013).

69 Lowet, E. et al. Input-dependent frequency modulation of cortical gamma oscillations shapes spatial synchronization and enables phase coding. PLoS Comput Biol 11, e1004072, doi:10.1371/journal.pcbi.1004072 (2015).

70 Dehghani, N. et al. Dynamic Balance of Excitation and Inhibition in Human and Monkey Neocortex. Sci Rep 6, 23176, doi:10.1038/srep23176 (2016).

71 Haider, B., Duque, A., Hasenstaub, A. R. \& McCormick, D. A. Neocortical network activity in vivo is generated through a dynamic balance of excitation and inhibition. J Neurosci 26, 4535-4545, doi:10.1523/JNEUROSCI.5297-05.2006 (2006).

72 Borg-Graham, L., Monier, C. \& Fregnac, Y. Voltage-clamp measurement of visually-evoked conductances with whole-cell patch recordings in primary visual cortex. J Physiol Paris 90, 185-188 (1996).

73 Okun, M. \& Lampl, I. Instantaneous correlation of excitation and inhibition during ongoing and sensory-evoked activities. Nat Neurosci 11, 535-537, doi:10.1038/nn.2105 (2008).

74 Bannai, H. et al. Bidirectional Control of Synaptic GABAAR Clustering by Glutamate and Calcium. Cell Rep 13, 2768-2780, doi:10.1016/j.celrep.2015.12.002 (2015).

75 Atallah, B. V. \& Scanziani, M. Instantaneous modulation of gamma oscillation frequency by balancing excitation with inhibition. Neuron 62, 566-577, doi:10.1016/j.neuron.2009.04.027 (2009).

76 Salinas, E. \& Sejnowski, T. J. Impact of correlated synaptic input on output firing rate and variability in simple neuronal models. J Neurosci 20, 6193-6209 (2000).

77 Ramón y Cajal, S., Pasik, P. \& Pasik, T. v. <1, $3>$ (Springer, Wien ; New York, 1999).

78 Abeles, M. Local cortical circuits : an electrophysiological study. (SpringerVerlag, 1982). 
79 Diesmann, M., Gewaltig, M. O. \& Aertsen, A. Stable propagation of synchronous spiking in cortical neural networks. Nature 402, 529-533, doi:10.1038/990101 (1999).

80 Abeles, M. Role of the cortical neuron: integrator or coincidence detector? Isr $J$ Med Sci 18, 83-92 (1982).

81 Friston, K. J. The labile brain. II. Transients, complexity and selection. Philos Trans R Soc Lond B Biol Sci 355, 237-252, doi:10.1098/rstb.2000.0561 (2000).

82 Ito, M., Tamura, H., Fujita, I. \& Tanaka, K. Size and position invariance of neuronal responses in monkey inferotemporal cortex. J Neurophysiol 73, 218-226 (1995).

83 Bosman, C. A. et al. Attentional stimulus selection through selective synchronization between monkey visual areas. Neuron $\mathbf{7 5}, 875-888$, doi:10.1016/j.neuron.2012.06.037 (2012).

84 Grun, S., Diesmann, M. \& Aertsen, A. Unitary events in multiple single-neuron spiking activity: 1. Detection and significance. Neural Computation 14, 43-80, doi:Doi 10.1162/089976602753284455 (2002).

85 Veit, J., Hakim, R., Jadi, M. P., Sejnowski, T. J. \& Adesnik, H. Cortical gamma band synchronization through somatostatin interneurons. Nat Neurosci, doi:10.1038/nn.4562 (2017).

86 Singer, W. \& Gray, C. M. Visual feature integration and the temporal correlation hypothesis. Annu Rev Neurosci 18, 555-586, doi:10.1146/annurev.ne.18.030195.003011 (1995).

87 Bastos, A. M., Vezoli, J. \& Fries, P. Communication through coherence with inter-areal delays. Curr Opin Neurobiol 31, 173-180, doi:10.1016/j.conb.2014.11.001 (2015).

88 van Kerkoerle, T. et al. Alpha and gamma oscillations characterize feedback and feedforward processing in monkey visual cortex. Proc Natl Acad Sci U S A 111, 14332-14341, doi:10.1073/pnas.1402773111 (2014).

89 Canolty, R. T. et al. High gamma power is phase-locked to theta oscillations in human neocortex. Science 313, 1626-1628, doi:10.1126/science.1128115 (2006).

90 Ito, J. et al. Whisker barrel cortex delta oscillations and gamma power in the awake mouse are linked to respiration. Nature Communications $\mathbf{5}$, doi:10.1038/ncomms4572 (2014).

91 Roopun, A. K. et al. Temporal Interactions between Cortical Rhythms. Front Neurosci 2, 145-154, doi:10.3389/neuro.01.034.2008 (2008). 
92 Heck, D. H. et al. Breathing as a Fundamental Rhythm of Brain Function. Front Neural Circuits 10, 115, doi:10.3389/fncir.2016.00115 (2016).

93 Friston, K. \& Kiebel, S. Predictive coding under the free-energy principle. Philos Trans R Soc Lond B Biol Sci 364, 1211-1221, doi:10.1098/rstb.2008.0300 (2009).

94 Bastos, A. M. et al. Canonical microcircuits for predictive coding. Neuron 76, 695-711, doi:10.1016/j.neuron.2012.10.038 (2012).

95 Thomas \& Anderson, J. F. Treatise on man. (Prentice-Hall, 1962).

96 Salinas, E. \& Sejnowski, T. J. Correlated neuronal activity and the flow of neural information. Nat Rev Neurosci 2, 539-550, doi:10.1038/35086012 (2001).

97 Nunez, A. \& Malmierca, E. Corticofugal modulation of sensory information. Adv Anat Embryol Cell Biol 187, 1 p following table of contents, 1-74 (2007).

98 Wimmer, R. D. et al. Thalamic control of sensory selection in divided attention. Nature 526, 705-709, doi:10.1038/nature15398 (2015).

99 Markov, N. T. et al. Anatomy of hierarchy: feedforward and feedback pathways in macaque visual cortex. $J$ Comp Neurol 522, 225-259, doi:10.1002/cne.23458 (2014).

100 Felleman, D. J. \& Van Essen, D. C. Distributed hierarchical processing in the primate cerebral cortex. Cerebral cortex 1, 1-47 (1991).

101 Lachaux, J. P., Rodriguez, E., Martinerie, J. \& Varela, F. J. Measuring phase synchrony in brain signals. Hum Brain Mapp 8, 194-208 (1999).

102 Freeman, W. J. Hilbert transform for brain waves. Scholarpedia 2, 1338 (2007).

103 Bruns, A. Fourier-, Hilbert- and wavelet-based signal analysis: are they really different approaches? J Neurosci Methods 137, 321-332, doi:10.1016/j.jneumeth.2004.03.002 (2004).

104 Le Van Quyen, M. et al. Comparison of Hilbert transform and wavelet methods for the analysis of neuronal synchrony. J Neurosci Methods 111, 83-98 (2001).

105 Lowet, E., Roberts, M. J., Bonizzi, P., Karel, J. \& De Weerd, P. Quantifying Neural Oscillatory Synchronization: A Comparison between Spectral Coherence and Phase-Locking Value Approaches. PLoS One 11, e0146443, doi:10.1371/journal.pone.0146443 (2016).

106 Allefeld, C. Phase synchronization analysis of event-related brain potentials in language processing, (2004). 
107 Berens, P. CircStat: A MATLAB Toolbox for Circular Statistics. Journal of Statistical Software; Vol 1, Issue 10 (2009), doi:10.18637/jss.v031.i10 (2009).

108 Maris, E. \& Oostenveld, R. Nonparametric statistical testing of EEG- and MEGdata. $J$ Neurosci Methods 164, 177-190, doi:10.1016/j.jneumeth.2007.03.024 (2007).

109 Grün, S., Borgelt, C., Gerstein, G., Louis, S. \& Diesmann, M. Selecting appropriate surrogate methods for spike correlation analysis. BMC Neuroscience 11, O15-O15, doi:10.1186/1471-2202-11-S1-O15 (2010).

110 Agmon, A. A novel, jitter-based method for detecting and measuring spike synchrony and quantifying temporal firing precision. Neural Syst Circuits 2, 5, doi:10.1186/2042-1001-2-5 (2012).

111 Dvorak, D. \& Fenton, A. A. Toward a proper estimation of phase-amplitude coupling in neural oscillations. J Neurosci Methods 225, 42-56, doi:10.1016/j.jneumeth.2014.01.002 (2014).

112 Moore, B. R. A Modification of the Rayleigh Test for Vector Data. Biometrika 67, 175-180, doi:10.2307/2335330 (1980).

113 Womelsdorf, T. et al. Modulation of neuronal interactions through neuronal synchronization. Science 316, 1609-1612, doi:10.1126/science.1139597 (2007).

114 Henrie, J. A. \& Shapley, R. LFP power spectra in V1 cortex: the graded effect of stimulus contrast. J Neurophysiol 94, 479-490, doi:10.1152/jn.00919.2004 (2005).

115 Roberts, M. J. et al. Robust gamma coherence between macaque V1 and V2 by dynamic frequency matching. Neuron 78, 523-536, doi:10.1016/j.neuron.2013.03.003 (2013).

116 Nase, G., Singer, W., Monyer, H. \& Engel, A. K. Features of neuronal synchrony in mouse visual cortex. $J$ Neurophysiol 90, 1115-1123, doi:10.1152/jn.00480.2002 (2003).

117 Bachatene, L. et al. Functional synchrony and stimulus selectivity of visual cortical units: Comparison between cats and mice. Neuroscience 337, 331-338, doi:10.1016/j.neuroscience.2016.09.030 (2016).

118 Kohn, A. \& Smith, M. A. Stimulus dependence of neuronal correlation in primary visual cortex of the macaque. $J$ Neurosci 25, 3661-3673, doi:10.1523/JNEUROSCI.5106-04.2005 (2005).

119 Usher, M. \& Donnelly, N. Visual synchrony affects binding and segmentation in perception. Nature 394, 179-182, doi:10.1038/28166 (1998). 
120 Castelo-Branco, M., Neuenschwander, S. \& Singer, W. Synchronization of visual responses between the cortex, lateral geniculate nucleus, and retina in the anesthetized cat. J Neurosci 18, 6395-6410 (1998).

121 Saleem, A. B. et al. Subcortical Source and Modulation of the Narrowband Gamma Oscillation in Mouse Visual Cortex. Neuron 93, 315-322, doi:10.1016/j.neuron.2016.12.028 (2017).

122 Koepsell, K. et al. Retinal oscillations carry visual information to cortex. Front Syst Neurosci 3, 4, doi:10.3389/neuro.06.004.2009 (2009).

123 Bryant, J. L., Roy, S. \& Heck, D. H. A technique for stereotaxic recordings of neuronal activity in awake, head-restrained mice. J Neurosci Methods 178, 75-79, doi:10.1016/j.jneumeth.2008.11.014 (2009).

124 Niell, C. M. \& Stryker, M. P. Highly selective receptive fields in mouse visual cortex. $J$ Neurosci 28, 7520-7536, doi:10.1523/JNEUROSCI.0623-08.2008 (2008).

125 Brainard, D. H. The Psychophysics Toolbox. Spat Vis 10, 433-436 (1997).

126 Pelli, D. G. The VideoToolbox software for visual psychophysics: transforming numbers into movies. Spat Vis 10, 437-442 (1997).

127 Vinck, M., van Wingerden, M., Womelsdorf, T., Fries, P. \& Pennartz, C. M. The pairwise phase consistency: a bias-free measure of rhythmic neuronal synchronization. Neuroimage 51, 112-122, doi:10.1016/j.neuroimage.2010.01.073 (2010).

128 MacLean, J. N., Fenstermaker, V., Watson, B. O. \& Yuste, R. A visual thalamocortical slice. Nat Methods 3, 129-134, doi:10.1038/nmeth849 (2006).

129 Ray, S. \& Maunsell, J. H. Different origins of gamma rhythm and high-gamma activity in macaque visual cortex. PLoS Biol 9, e1000610, doi:10.1371/journal.pbio.1000610 (2011).

130 Storchi, R. et al. Modulation of Fast Narrowband Oscillations in the Mouse Retina and dLGN According to Background Light Intensity. Neuron 93, 299-307, doi:10.1016/j.neuron.2016.12.027 (2017).

131 Belitski, A. et al. Low-frequency local field potentials and spikes in primary visual cortex convey independent visual information. $J$ Neurosci 28, 5696-5709, doi:10.1523/JNEUROSCI.0009-08.2008 (2008).

132 Jones, E. G. Thalamic circuitry and thalamocortical synchrony. Philos Trans $R$ Soc Lond B Biol Sci 357, 1659-1673, doi:10.1098/rstb.2002.1168 (2002). 
133 Wang, H. P., Spencer, D., Fellous, J. M. \& Sejnowski, T. J. Synchrony of thalamocortical inputs maximizes cortical reliability. Science 328, 106-109, doi:10.1126/science.1183108 (2010).

134 Sherman, S. M. \& Guillery, R. W. The role of the thalamus in the flow of information to the cortex. Philos Trans R Soc Lond B Biol Sci 357, 1695-1708, doi:10.1098/rstb.2002.1161 (2002).

135 Jones, E. G. The thalamic matrix and thalamocortical synchrony. Trends Neurosci 24, 595-601 (2001).

136 Schoffelen, J. M., Poort, J., Oostenveld, R. \& Fries, P. Selective movement preparation is subserved by selective increases in corticomuscular gamma-band coherence. J Neurosci 31, 6750-6758, doi:10.1523/JNEUROSCI.4882-10.2011 (2011).

137 Dugue, L., Marque, P. \& VanRullen, R. The phase of ongoing oscillations mediates the causal relation between brain excitation and visual perception. $J$ Neurosci 31, 11889-11893, doi:10.1523/JNEUROSCI.1161-11.2011 (2011).

138 Bieri, K. W., Bobbitt, K. N. \& Colgin, L. L. Slow and fast gamma rhythms coordinate different spatial coding modes in hippocampal place cells. Neuron 82, 670-681, doi:10.1016/j.neuron.2014.03.013 (2014).

139 Buckner, R. L. The cerebellum and cognitive function: 25 years of insight from anatomy and neuroimaging. Neuron $\mathbf{8 0}, 807-815$, doi:10.1016/j.neuron.2013.10.044 (2013).

140 Tedesco, A. M. et al. The cerebellar cognitive profile. Brain : a journal of neurology 134, 3672-3686, doi:10.1093/brain/awr266 (2011).

141 Strick, P. L., Dum, R. P. \& Fiez, J. A. Cerebellum and nonmotor function. Annu Rev Neurosci 32, 413-434, doi:10.1146/annurev.neuro.31.060407.125606 (2009).

142 Schmahmann, J. D. \& Caplan, D. Cognition, emotion and the cerebellum. Brain : a journal of neurology 129, 290-292 (2006).

143 Dean, H. L., Hagan, M. A. \& Pesaran, B. Only coherent spiking in posterior parietal cortex coordinates looking and reaching. Neuron 73, 829-841, doi:10.1016/j.neuron.2011.12.035 (2012).

144 Jacobson, G. A., Rokni, D. \& Yarom, Y. A model of the olivo-cerebellar system as a temporal pattern generator. Trends Neurosci. 31, 617-625 (2008).

145 Grammont, F. \& Riehle, A. Spike synchronization and firing rate in a population of motor cortical neurons in relation to movement direction and reaction time. Biol.Cybern. 88, 360-373 (2003). 
146 Baker, S. N., Spinks, R., Jackson, A. \& Lemon, R. N. Synchronization in monkey motor cortex during a precision grip task. I. Task-dependent modulation in singleunit synchrony. Journal of Neurophysiology 85, 869-885 (2001).

147 Mathewson, K. E. et al. Making waves in the stream of consciousness: entraining oscillations in EEG alpha and fluctuations in visual awareness with rhythmic visual stimulation. Journal of cognitive neuroscience 24, 2321-2333, doi:10.1162/jocn_a_00288(2012).

148 Rizzuto, D. S., Madsen, J. R., Bromfield, E. B., Schulze-Bonhage, A. \& Kahana, M. J. Human neocortical oscillations exhibit theta phase differences between encoding and retrieval. Neuroimage 31, 1352-1358, doi:10.1016/j.neuroimage.2006.01.009 (2006).

149 Hirabayashi, T. \& Miyashita, Y. Dynamically modulated spike correlation in monkey inferior temporal cortex depending on the feature configuration within a whole object. J.Neurosci 25, 10299-10307 (2005).

150 Ward, L. M. Synchronous neural oscillations and cognitive processes. Trends Cogn Sci. 7, 553-559 (2003).

151 Steinmetz, P. N. et al. Attention modulates synchonized neuronal firing in primate somatosenroy cortex. Nature 404, 187-190 (2000).

152 Riehle, A., GrÅn, S., Diesmann, M. \& Aertsen, A. Spike synchronization and rate modulation differentially involved in motor cortical function. Science 278, 19501953 (1997).

153 Ivry, R. B., Keele, S. W. \& Diener, H. C. Dissociation of the lateral and medial cerebellum in movement timing and movement execution. Exp Brain Res 73, 167180 (1988).

154 Becker, W. J., Morrice, B. L., Clark, A. W. \& Lee, R. G. Multi-joint reaching movements and eye-hand tracking in cerebellar incoordination: Investigation of a patient with complete loss of Purkinje cells. Can.J.Neurol.Sci. 18, 476-487 (1991).

155 Berridge, K. C. \& Whishaw, I. Q. Cortex, striatum and cerebellum: Control of serial order in a grooming sequence. Exp Brain Res 90, 275-290 (1992).

156 Brown, S. H., Kessler, K. R., Hefter, H., Cooke, J. D. \& Freund, H. J. Role of the cerebellum in visuomotor coordination. I. Delayed eye and arm initiation in patients with mild cerebellar ataxia. Exp Brain Res 94, 478-488 (1993).

157 Welsh, J. P., Lang, E. J., Sugihara, I. \& Llin†s, R. Dynamic organization of motor control within the olivocerebellar system. Nature 374, 453-457 (1995). 
158 Diener, H. C. et al. The coordination of posture and voluntary movement in patients with cerebellar dysfunction. Mov Disord. 7, 14-22 (1992).

159 Topka, H., Konczak, J., Schneider, K., Boose, A. \& Dichgans, J. Multijoint arm movements in cerebellar ataxia: Abnormal control of movement dynamics. Exp Brain Res 119, 493-503 (1998).

160 Diener, H. C., Hore, J., Ivry, R. \& Dichgans, J. Cerebellar dysfunction of movement and perception. Can.J.Neurol.Sci. 20 Suppl. 3, S62-S69 (1993).

161 Penhune, V. B., Zattore, R. J. \& Evans, A. C. Cerebellar contributions to motor timing: a PET study of auditory and visual rhythm reproduction. J.Cogn Neurosci 10, 752-765 (1998).

162 Rauschecker, J. P. Neural encoding and retrieval of sound sequences. Ann.N.Y.Acad.Sci. 1060, 125-135 (2005).

163 Leggio, M. \& Molinari, M. Cerebellar sequencing: a trick for predicting the future. Cerebellum 14, 35-38, doi:10.1007/s12311-014-0616-x (2015).

164 Mathiak, K., Hertrich, I., Grodd, W. \& Ackermann, H. Cerebellum and speech perception: a functional magnetic resonance imaging study. Journal of cognitive neuroscience 14, 902-912, doi:10.1162/089892902760191126 (2002).

165 Perrett, S. P., Ruiz, B. P. \& Mauk, M. D. Cerebellar cortex lesions disrupt learning-dependent timing of conditioned eyelid responses. J.Neurosci 13, 17081718 (1993).

166 Perrett, S. P. \& Mauk, M. D. Extinction of conditioned eyelid responses requires the anterior lobe of cerebellar cortex. J.Neurosci 15, 2074-2080 (1995).

167 Kalmbach, B. E., Ohyama, T., Kreider, J. C., Riusech, F. \& Mauk, M. D. Interactions between prefrontal cortex and cerebellum revealed by trace eyelid conditioning. Learn Mem 16, 86-95, doi:16/1/86 [pii]

10.1101/lm.1178309 (2009).

168 Benchenane, K. et al. Coherent theta oscillations and reorganization of spike timing in the hippocampal- prefrontal network upon learning. Neuron 66, 921 936, doi:10.1016/j.neuron.2010.05.013 (2010).

169 Gordon, J. A. Oscillations and hippocampal-prefrontal synchrony. Curr Opin Neurobiol 21, 486-491, doi:10.1016/j.conb.2011.02.012 (2011).

170 Galvin, C. \& Ninan, I. Regulation of the mouse medial prefrontal cortical synapses by endogenous estradiol. Neuropsychopharmacology 39, 2086-2094, doi:10.1038/npp.2014.56 (2014). 
171 Buzsaki, G. Hippocampal sharp wave-ripple: A cognitive biomarker for episodic memory and planning. Hippocampus 25, 1073-1188, doi:10.1002/hipo.22488 (2015).

172 Paxinos, G. \& Franklin, K. B. J. The Mouse Brain in Stereotaxic Coordinates. Vol. 2 (Academic Press, 2001).

173 Thach, W. T. Discharge of cerebellar neurons related to two maintained postures and two prompt movements. II.Purkinje cell output and input. Journal of Neurophysiology 33, 537-547 (1970).

174 Braitenberg, V., Heck, D. H. \& Sultan, F. The detection and generation of sequences as a key to cerebellar function. Experiments and theory. Behav.Brain Sci. 20, 229-245 (1997).

175 Heck, D. H. Sequential input to guinea pig cerebellar cortex in vitro strongly affects Purkinje cells via parallel fibers. Naturwissenschaften 82, 201-203 (1995).

176 Heck, D. H. \& Sultan, F. Cerebellar structure and function: making sense of parallel fibers. Hum.Mov.Sci. 21, 411-421 (2002).

177 Lefort, J. M., Rochefort, C., Rondi-Reig, L., Group of, L. R. R. i. m. o. B.-P. L. \& Foundation, E. N. P. Cerebellar contribution to spatial navigation: new insights into potential mechanisms. Cerebellum 14, 59-62, doi:10.1007/s12311-015-06530 (2015).

178 Spellman, T. et al. Hippocampal-prefrontal input supports spatial encoding in working memory. Nature 522, 309-314, doi:10.1038/nature14445 (2015).

179 Popa, D. et al. Functional role of the cerebellum in gamma-band synchronization of the sensory and motor cortices. $J$ Neurosci 33, 6552-6556, doi:10.1523/JNEUROSCI.5521-12.2013 (2013).

180 Braitenberg, V. Functional Interpretation of Cerebellar Histology. Nature 190, 539-540 (1961).

181 Ivry, R. B., Spencer, R. M., Zelaznik, H. N. \& Diedrichsen, J. The cerebellum and event timing. Ann N Y Acad Sci 978, 302-317 (2002).

182 Adrian, E. D. Olfactory reactions in the brain of the hedgehog. $J$ Physiol 100, 459-473 (1942).

183 Phillips, M. E., Sachdev, R. N., Willhite, D. C. \& Shepherd, G. M. Respiration drives network activity and modulates synaptic and circuit processing of lateral inhibition in the olfactory bulb. $J$ Neurosci 32, 85-98, doi:10.1523/JNEUROSCI.4278-11.2012 (2012). 
184 Martin, C. \& Ravel, N. Beta and gamma oscillatory activities associated with olfactory memory tasks: different rhythms for different functional networks? Front Behav Neurosci 8, 218, doi:10.3389/fnbeh.2014.00218 (2014).

185 Rojas-Libano, D. \& Kay, L. M. Olfactory system gamma oscillations: the physiological dissection of a cognitive neural system. Cogn Neurodyn 2, 179-194, doi:10.1007/s11571-008-9053-1 (2008).

186 Buonviso, N. et al. Rhythm sequence through the olfactory bulb layers during the time window of a respiratory cycle. The European journal of neuroscience 17, 1811-1819 (2003).

187 Manns, I. D., Alonso, A. \& Jones, B. E. Rhythmically discharging basal forebrain units comprise cholinergic, GABAergic, and putative glutamatergic cells. $J$ Neurophysiol 89, 1057-1066, doi:10.1152/jn.00938.2002 (2003).

188 Carmichael, J. E., Gmaz, J. M. \& van der Meer, M. A. A. Gamma Oscillations in the Rat Ventral Striatum Originate in the Piriform Cortex. J Neurosci 37, 79627974, doi:10.1523/JNEUROSCI.2944-15.2017 (2017).

189 Nguyen Chi, V. et al. Hippocampal Respiration-Driven Rhythm Distinct from Theta Oscillations in Awake Mice. J Neurosci 36, 162-177, doi:10.1523/JNEUROSCI.2848-15.2016 (2016).

190 Yanovsky, Y., Ciatipis, M., Draguhn, A., Tort, A. B. \& Brankack, J. Slow oscillations in the mouse hippocampus entrained by nasal respiration. $J$ Neurosci 34, 5949-5964, doi:10.1523/JNEUROSCI.5287-13.2014 (2014).

191 Biskamp, J., Bartos, M. \& Sauer, J. F. Organization of prefrontal network activity by respiration-related oscillations. Sci Rep 7, 45508, doi:10.1038/srep45508 (2017).

192 Lee, S. H. \& Dan, Y. Neuromodulation of brain states. Neuron 76, 209-222, doi:10.1016/j.neuron.2012.09.012 (2012).

193 van der Meer, M. A. et al. Integrating early results on ventral striatal gamma oscillations in the rat. Front Neurosci 4, 300, doi:10.3389/fnins.2010.00300 (2010).

194 Mas-Herrero, E. \& Marco-Pallares, J. Theta oscillations integrate functionally segregated sub-regions of the medial prefrontal cortex. Neuroimage 143, 166-174, doi:10.1016/j.neuroimage.2016.08.024 (2016).

195 Wirt, R. A. \& Hyman, J. M. Integrating Spatial Working Memory and Remote Memory: Interactions between the Medial Prefrontal Cortex and Hippocampus. Brain Sci 7, doi:10.3390/brainsci7040043 (2017). 
196 Groenewegen, H. J. \& Uylings, H. B. The prefrontal cortex and the integration of sensory, limbic and autonomic information. Progress in brain research 126, 3-28, doi:10.1016/S0079-6123(00)26003-2 (2000).

197 Heck, D. H. et al. Breathing as a fundamental rhythm of brain function. Frontiers in Neural Circuits 10 (2017).

198 McAfee, S. S. et al. Minimally invasive highly precise monitoring of respiratory rhythm in the mouse using an epithelial temperature probe. J Neurosci Methods 263, 89-94, doi:10.1016/j.jneumeth.2016.02.007 (2016).

199 Shusterman, R., Smear, M. C., Koulakov, A. A. \& Rinberg, D. Precise olfactory responses tile the sniff cycle. Nat Neurosci 14, 1039-1044, doi:10.1038/nn.2877 (2011).

200 Kleinfeld, D., Deschenes, M., Wang, F. \& Moore, J. D. More than a rhythm of life: breathing as a binder of orofacial sensation. Nature Neuroscience 17, 647651, doi:10.1038/nn.3693 (2014).

201 Reisert, J. et al. Comparing thoracic and intra-nasal pressure transients to monitor active odor sampling during odor-guided decision making in the mouse. $J$ Neurosci Meth 221, 8-14, doi:10.1016/j.jneumeth.2013.09.006 (2014).

202 Smear, M., Shusterman, R., O'Connor, R., Bozza, T. \& Rinberg, D. Perception of sniff phase in mouse olfaction. Nature 479, 397-U149, doi:10.1038/nature10521 (2011).

203 Batschelet, E. Circular statistics in biology. (Academic Press, 1981).

204 Lim, R. Z., Marcus J.; Milton, Phillipa-Louise; Chan, Siow Teng; Tan, Jean L. ; Dickinson, Hayley; Murphy, Sean V.; Jenkin, Graham; Wallace,Euan M. Measuring Respiratory Function in Mice Using Unrestrained Whole-body Plethysmography. Journal of Visual Experiments, doi:10.3791/51755 (2014).

205 Youngentob, S. L. A method for the rapid automated assessment of olfactory function. Chem Senses 30, 219-229, doi:10.1093/chemse/bji017 (2005).

206 Hegoburu, C. et al. The RUB cage: respiration-ultrasonic vocalizations-behavior acquisition setup for assessing emotional memory in rats. Front Behav Neurosci 5, doi:Artn 25

10.3389/Fnbeh.2011.00025 (2011).

207 Uchida, N. \& Mainen, Z. F. Speed and accuracy of olfactory discrimination in the rat. Nature Neuroscience 6, 1224-1229, doi:10.1038/nn1142 (2003).

208 Wesson, D. W. Sniffing Behavior Communicates Social Hierarchy. Current Biology 23, 575-580, doi:10.1016/j.cub.2013.02.012 (2013). 
209 Wesson, D. W., Donahou, T. N., Johnson, M. O. \& Wachowiak, M. Sniffing behavior of mice during performance in odor-guided tasks. Chem Senses 33, 581596, doi:10.1093/chemse/bjn029 (2008).

210 Urban, D. J. \& Roth, B. L. DREADDs (designer receptors exclusively activated by designer drugs): chemogenetic tools with therapeutic utility. Annual review of pharmacology and toxicology 55, 399-417, doi:10.1146/annurev-pharmtox010814-124803 (2015).

211 Zou, D. J., Greer, C. A. \& Firestein, S. Expression pattern of alpha CaMKII in the mouse main olfactory bulb. J Comp Neurol 443, 226-236 (2002).

212 Wallace, M. T., Ramachandran, R. \& Stein, B. E. A revised view of sensory cortical parcellation. Proc Natl Acad Sci U S A 101, 2167-2172, doi:10.1073/pnas.0305697101 (2004).

213 Buzsaki, G. \& Gage, F. H. The cholinergic nucleus basalis: a key structure in neocortical arousal. EXS 57, 159-171 (1989).

214 Buzsaki, G. et al. Nucleus basalis and thalamic control of neocortical activity in the freely moving rat. $J$ Neurosci 8, 4007-4026 (1988).

215 Lee, S. C., Patrick, S. L., Richardson, K. A. \& Connors, B. W. Two functionally distinct networks of gap junction-coupled inhibitory neurons in the thalamic reticular nucleus. J Neurosci 34, 13170-13182, doi:10.1523/JNEUROSCI.056214.2014 (2014).

216 Landisman, C. E. et al. Electrical synapses in the thalamic reticular nucleus. $J$ Neurosci 22, 1002-1009 (2002).

217 Smith, J. C., Ellenberger, H. H., Ballanyi, K., Richter, D. W. \& Feldman, J. L. Pre-Botzinger complex: a brainstem region that may generate respiratory rhythm in mammals. Science 254, 726-729 (1991).

218 Carstens, E., Leah, J., Lechner, J. \& Zimmermann, M. Demonstration of extensive brainstem projections to medial and lateral thalamus and hypothalamus in the rat. Neuroscience 35, 609-626 (1990).

219 Ahlsen, G. \& Lo, F. S. Projection of brain stem neurons to the perigeniculate nucleus and the lateral geniculate nucleus in the cat. Brain Res 238, 433-438 (1982).

220 Lee, A. M. et al. Identification of a brainstem circuit regulating visual cortical state in parallel with locomotion. Neuron 83, 455-466, doi:10.1016/j.neuron.2014.06.031 (2014).

221 Goard, M. \& Dan, Y. Basal forebrain activation enhances cortical coding of natural scenes. Nat Neurosci 12, 1444-1449, doi:10.1038/nn.2402 (2009). 
$222 \mathrm{Fu}$, Y. et al. A cortical circuit for gain control by behavioral state. Cell 156, 11391152, doi:10.1016/j.cell.2014.01.050 (2014).

223 Prusky, G. T., Alam, N. M., Beekman, S. \& Douglas, R. M. Rapid quantification of adult and developing mouse spatial vision using a virtual optomotor system. Invest Ophthalmol Vis Sci 45, 4611-4616, doi:10.1167/iovs.04-0541 (2004).

224 Lockmann, A. L., Laplagne, D. A., Leao, R. N. \& Tort, A. B. A RespirationCoupled Rhythm in the Rat Hippocampus Independent of Theta and Slow Oscillations. J Neurosci 36, 5338-5352, doi:10.1523/JNEUROSCI.3452-15.2016 (2016).

225 Malvache, A., Reichinnek, S., Villette, V., Haimerl, C. \& Cossart, R. Awake hippocampal reactivations project onto orthogonal neuronal assemblies. Science 353, 1280-1283, doi:10.1126/science.aaf3319 (2016).

226 Nicole, O. et al. Soluble amyloid beta oligomers block the learning-induced increase in hippocampal sharp wave-ripple rate and impair spatial memory formation. Sci Rep 6, 22728, doi:10.1038/srep22728 (2016).

227 van de Ven, G. M., Trouche, S., McNamara, C. G., Allen, K. \& Dupret, D. Hippocampal Offline Reactivation Consolidates Recently Formed Cell Assembly Patterns during Sharp Wave-Ripples. Neuron 92, 968-974, doi:10.1016/j.neuron.2016.10.020 (2016).

228 Jadhav, S. P., Kemere, C., German, P. W. \& Frank, L. M. Awake hippocampal sharp-wave ripples support spatial memory. Science 336, 1454-1458, doi:10.1126/science.1217230 (2012).

229 Singer, A. C., Carr, M. F., Karlsson, M. P. \& Frank, L. M. Hippocampal SWR activity predicts correct decisions during the initial learning of an alternation task. Neuron 77, 1163-1173, doi:10.1016/j.neuron.2013.01.027 (2013).

230 Pfeiffer, B. E. \& Foster, D. J. Autoassociative dynamics in the generation of sequences of hippocampal place cells. Science 349, 180-183, doi:10.1126/science.aaa9633 (2015).

231 Maingret, N., Girardeau, G., Todorova, R., Goutierre, M. \& Zugaro, M. Hippocampo-cortical coupling mediates memory consolidation during sleep. Nat Neurosci 19, 959-964, doi:10.1038/nn.4304 (2016).

232 Papale, A. E., Zielinski, M. C., Frank, L. M., Jadhav, S. P. \& Redish, A. D. Interplay between Hippocampal Sharp-Wave-Ripple Events and Vicarious Trial and Error Behaviors in Decision Making. Neuron 92, 975-982, doi:10.1016/j.neuron.2016.10.028 (2016). 
233 O'Neill, J., Boccara, C. N., Stella, F., Schoenenberger, P. \& Csicsvari, J.

Superficial layers of the medial entorhinal cortex replay independently of the hippocampus. Science 355, 184-188, doi:10.1126/science.aag2787 (2017).

234 Rothschild, G., Eban, E. \& Frank, L. M. A cortical-hippocampal-cortical loop of information processing during memory consolidation. Nat Neurosci 20, 251-259, doi:10.1038/nn.4457 (2017).

$235 \mathrm{Wu}, \mathrm{C}$. T., Haggerty, D., Kemere, C. \& Ji, D. Hippocampal awake replay in fear memory retrieval. Nat Neurosci 20, 571-580, doi:10.1038/nn.4507 (2017).

236 Logothetis, N. K. et al. Hippocampal-cortical interaction during periods of subcortical silence. Nature 491, 547-553, doi:10.1038/nature11618 (2012).

237 Leonard, T. K. \& Hoffman, K. L. Sharp-Wave Ripples in Primates Are Enhanced near Remembered Visual Objects. Curr Biol 27, 257-262, doi:10.1016/j.cub.2016.11.027 (2017).

238 Ramirez-Villegas, J. F., Logothetis, N. K. \& Besserve, M. Diversity of sharpwave-ripple LFP signatures reveals differentiated brain-wide dynamical events. Proc Natl Acad Sci U S A 112, E6379-6387, doi:10.1073/pnas.1518257112 (2015).

239 Liu, Y., McAfee, S. S. \& Heck, D. H. Hippocampal sharp-wave ripples in awake mice are entrained by respiration. Sci Rep 7, 8950, doi:10.1038/s41598-01709511-8 (2017).

240 Schlingloff, D., Kali, S., Freund, T. F., Hajos, N. \& Gulyas, A. I. Mechanisms of sharp wave initiation and ripple generation. J Neurosci 34, 11385-11398, doi:10.1523/JNEUROSCI.0867-14.2014 (2014).

241 Buzsaki, G., Leung, L. W. \& Vanderwolf, C. H. Cellular bases of hippocampal EEG in the behaving rat. Brain Res 287, 139-171 (1983).

242 Miles, R. \& Wong, R. K. Single neurones can initiate synchronized population discharge in the hippocampus. Nature 306, 371-373 (1983).

243 de la Prida, L. M., Huberfeld, G., Cohen, I. \& Miles, R. Threshold behavior in the initiation of hippocampal population bursts. Neuron 49, 131-142, doi:10.1016/j.neuron.2005.10.034 (2006).

244 Vandecasteele, M. et al. Optogenetic activation of septal cholinergic neurons suppresses sharp wave ripples and enhances theta oscillations in the hippocampus. Proc Natl Acad Sci U S A 111, 13535-13540, doi:10.1073/pnas.1411233111 (2014). 
245 Fang, J. et al. Data-driven analysis of functional brain interactions during free listening to music and speech. Brain Imaging Behav 9, 162-177, doi:10.1007/s11682-014-9293-0 (2015).

246 Rochet-Capellan, A. \& Fuchs, S. Changes in breathing while listening to read speech: the effect of reader and speech mode. Front Psychol 4, 906, doi:10.3389/fpsyg.2013.00906 (2013).

247 Seppala, E. M. et al. Breathing-based meditation decreases posttraumatic stress disorder symptoms in U.S. military veterans: a randomized controlled longitudinal study. J Trauma Stress 27, 397-405, doi:10.1002/jts.21936 (2014).

248 Heck, D. H. et al. Cortical rhythms are modulated by respiration. BioRxive, doi:http://dx.doi.org/10.1101/049007. (2016).

249 Freeman, W. J. A cinematographic hypothesis of cortical dynamics in perception. Int J Psychophysiol 60, 149-161, doi:10.1016/j.ijpsycho.2005.12.009 (2006).

250 Kozma, R. \& Freeman, W. J. in Cognitive Phase Transitions in the Cerebral Cortex - Enhancing the Neuron Doctrine by Modeling Neural Fields (eds Robert Kozma \& Walter J. Freeman) 71-78 (Springer International Publishing, 2016).

251 Kozma, R. \& Puljic, M. Random graph theory and neuropercolation for modeling brain oscillations at criticality. Curr Opin Neurobiol 31, 181-188, doi:10.1016/j.conb.2014.11.005 (2015).

252 Zelano, C. et al. Nasal Respiration Entrains Human Limbic Oscillations and Modulates Cognitive Function. J Neurosci 36, 12448-12467, doi:10.1523/JNEUROSCI.2586-16.2016 (2016).

253 Brown, P., Salenius, S., Rothwell, J. C. \& Hari, R. Cortical correlate of the Piper rhythm in humans. J Neurophysiol 80, 2911-2917 (1998).

254 Lopes da Silva, F. EEG and MEG: relevance to neuroscience. Neuron 80, 11121128, doi:10.1016/j.neuron.2013.10.017 (2013).

255 Womelsdorf, T. et al. Orientation selectivity and noise correlation in awake monkey area V1 are modulated by the gamma cycle. Proc Natl Acad Sci U S A 109, 4302-4307, doi:10.1073/pnas.1114223109 (2012).

256 Jones, M. W. \& Wilson, M. A. Theta rhythms coordinate hippocampal-prefrontal interactions in a spatial memory task. PLoS Biol 3, e402, doi:10.1371/journal.pbio.0030402 (2005). 


\section{VITA}

Samuel Stuart McAfee was born in Fremont, California in 1987. He earned his Bachelors of Science in Neuroscience from the University of Texas at Dallas in Richardson, Texas in the Summer of 2010. After his undergraduate education, he worked as a Diagnostic Technician and Research Assistant for Neurology departments in hospitals in both Austin, Texas and Memphis, Tennessee. In 2013, he was accepted to the graduate program in Neuroscience within the school of Integrated Biomedical Sciences at the University of Tennessee Health Science Center in Memphis, Tennessee. He will be awarded his PhD in December of 2017. 